\title{
WestVirginiaUniversity
}

THE RESEARCH REPOSITORY @ WVU

Graduate Theses, Dissertations, and Problem Reports

2015

\section{The Diverse Relationship of Kruppel-Like Factors 4 and 5 in Breast Cancer}

Mark K. Farrugia

Follow this and additional works at: https://researchrepository.wvu.edu/etd

\section{Recommended Citation}

Farrugia, Mark K., "The Diverse Relationship of Kruppel-Like Factors 4 and 5 in Breast Cancer" (2015). Graduate Theses, Dissertations, and Problem Reports. 5581.

https://researchrepository.wvu.edu/etd/5581

This Dissertation is protected by copyright and/or related rights. It has been brought to you by the The Research Repository @ WVU with permission from the rights-holder(s). You are free to use this Dissertation in any way that is permitted by the copyright and related rights legislation that applies to your use. For other uses you must obtain permission from the rights-holder(s) directly, unless additional rights are indicated by a Creative Commons license in the record and/ or on the work itself. This Dissertation has been accepted for inclusion in WVU Graduate Theses, Dissertations, and Problem Reports collection by an authorized administrator of The Research Repository @ WVU.

For more information, please contact researchrepository@mail.wvu.edu. 


\title{
The Diverse Relationship of Krüppel-Like Factors 4 and 5 in Breast Cancer
}

\author{
Mark K. Farrugia \\ Dissertation submitted to the School of Medicine at West Virginia University \\ In partial fulfillment of the requirements for the degree of
}

Doctor of Philosophy

in

Cancer Cell Biology

Michael Schaller, Ph.D., Chair

Alexey Ivanov, Ph.D.

Laura F. Gibson, Ph.D.

Peter Stoilov, Ph.D.

Soumit Basu, M.D., Ph.D.

J. Michael Ruppert, M.D., Ph.D., Mentor

Cancer Cell Biology Program

Morgantown, West Virginia

2015

Key Words: KLF4, KLF5, HER2, therapeutic resistance, lapatinib, apoptosis, breast cancer Copyright 2015 Mark Farrugia 


\title{
ABSTRACT \\ The Relationship of Kruppel-like Factors 4 and 5 in Breast Cancer
}

\author{
Mark K. Farrugia
}

Kruppel-like factors (KLFs) are transcription factors that participate in numerous biological functions, mainly impacting cell fate and differentiation. KLFs 4 and 5 (KLF4/5) are two highly studied members of this family, implicated to have diverse roles in both normal and abnormal physiology. KLF4 is primarily expressed in post-mitotic, differentiated epithelium and consistent with this observation, KLF4 exerts suppressive effects on cellular growth. Paradoxically, KLF4 can promote malignant features in both squamous cell carcinoma of the skin and breast cancer. We have attempted to better understand the nature of KLF4 in cancer via its relationship with KLF5. Although the two are believed to exert opposing influences on epithelial differentiation and growth, both have important roles in breast tumorigenesis.

Previously, KLFs 2, 4, and 5 were shown to function redundantly in the maintenance of the stem cell phenotype in embryonic stem cells, a context often informative to cancer. Despite individual reports detailing oncogenic effects of KLFs 4 and 5 in breast cancer, currently no relationship between the two KLFs has been described in this disease.

In Chapter 2 of this dissertation, we identified vital roles for KLF4/5 involving resistance to HER2-targeted therapies in breast cancer. Using comprehensive gene expression analysis of primary tumors from human and murine model systems, we identified a positive correlation between KLF4/5 transcript abundance, particularly in HER2-enriched tumors. Incorporating these in vivo observations into phenotypic studies revealed that KLF4/5 are critical in the cellular response to HER2 targeted therapies. The expression and activity of these KLFs was induced following HER2-inhibition, facilitating enhanced resistance to these treatments. Even in drugnaïve cells, depletion of KLF4/5 resulted in reduced anoikis resistance, colony formation, and xenograft formation. These defects were attributed to diminished levels of the anti-apoptotic factors BCL-XL and MCL1in KLF-deficient cells. In the absence of drug, KLF4/5 impacted the basal levels of BCL-XL and MCL1. Similar to KLF4/5, MCL1 and BCL-XL expression was also induced following HER2-inhibition and suppression of KLF4/5 blunted this effect. Consistent with their role in drug resistance, KLF4/5 transcript levels stratify distant metastasis-free survival in patients, with elevated levels of these factors associated with poor outcome.

Although KLF4/5 have overlapping functions in drug resistance, they can exert distinct and opposing influences as well. Chapter 3 further explores this relationship in breast cancer and attempts to address how KLF4/5 interact in alternative effector pathways. Here, we demonstrate non-redundant functions for the KLFs in breast cancer. Several studies have documented 
opposing roles for KLF4/5 on cell proliferation, with KLF4 exerting inhibitory effects and KLF5 promoting cell growth. Here we demonstrate that the proliferative phenotype of KLF4-depleted cells requires KLF5 expression, such that reduction of KLF5 abolishes the enhanced growth of these cells. These observations partially explain how KLF4 can dually act as an oncogene despite its growth-inhibitory effects. Furthermore, we explore distinct functions of these KLFs as well. In KLF4-depleted MDA-MB-231 cells, KLF4 was the only core KLF able to rescue the specific deficits resulting from its depletion. In addition, we elaborated on a previously described association between KLF4 and Notch1 signaling in breast cancer. We found KLF4 to transcriptionally participate in canonical Notch1 signaling, binding to and upregulating several target genes of this pathway including Notch1, Hes5 and Hey1. Moreover, KLF5 did not significantly contribute to the Notch1 pathway in this setting, further supporting non-redundant roles for the KLFs in certain effector pathways. Expanding on this relationship between KLF4 and Notch1, we compared ChIP-seq datasets for these respective genes in a stem cell context finding a significant overlap in many signaling cascades known to be important in tumorigenesis such as Wnt and Ras.

In summary, the relationship between KLFs KLF4/5 in breast carcinoma contributes to our understanding on how the genes participate in response to therapy and the cancer process. In future functional and translational studies, tandem analysis of both genes may provide additional insight than investigating these genes individually. 


\section{Dedication}

This dissertation is dedicated to my wife Ali, my family, friends and all of those who believed in me. Your support went further than you can ever imagine. 


\section{Acknowledgements}

I would like to acknowledge my extraordinary wife Alison Vargovich, $\mathrm{PhD}$. Your continued support and inspiration motivated and comforted me throughout many of the challenges faced during my training. To my family and friends, you gave me perspective outside of the academic microcosm, allowing me to relax and see the bigger picture.

Thank you to my advisor J. Michael Ruppert for his mentorship and leadership. In addition, the members of my lab have helped me tremendously. I'd like to express gratitude to Wentao Deng, Chen-Chung Lin, Sriganesh Sharma, Daniel Vanderbilt, Mike McKinstry, and Jackie Metheny for everything they contributed to my training and this project.

Thank you to the faculty and graduate students of the Cancer Cell Biology program, especially the members of my dissertation committee Drs. Schaller, Gibson, Ivanov, Stoilov, and Basu. In particular, I would like to thank the members of Gibson and Frisch lab for technical support and advice.

Lastly, many thanks to the West Virginia University $\mathrm{MD} / \mathrm{PhD}$ program for not only financial support, but for mentorship of both the faculty and students. 


\section{Table of Contents}

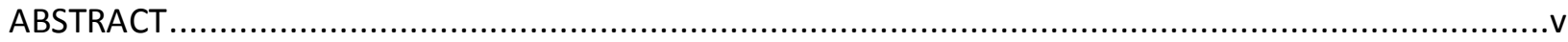

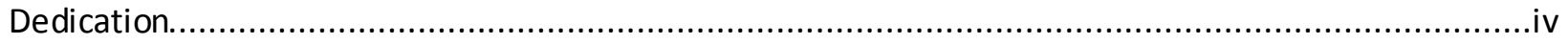

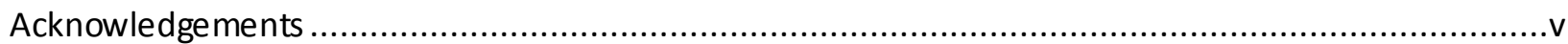

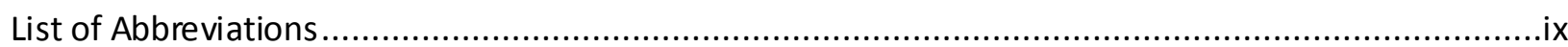

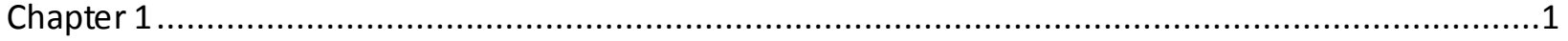

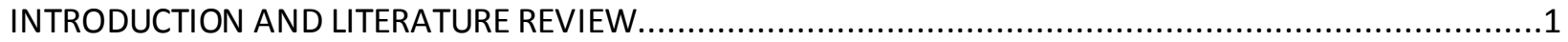

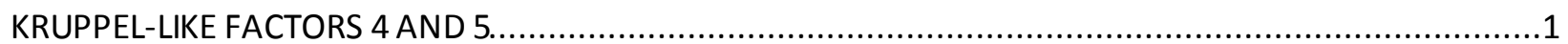

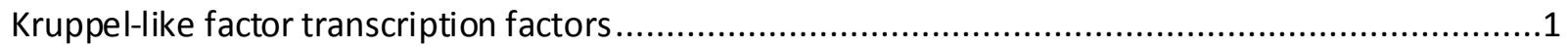

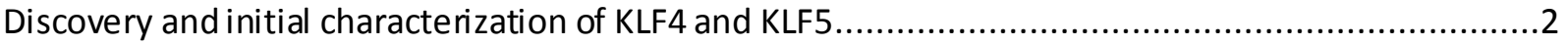

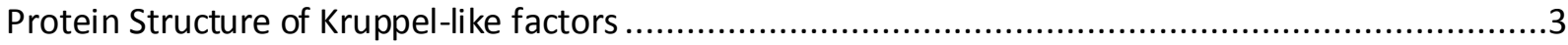

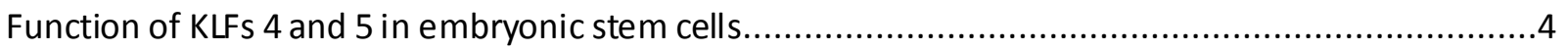

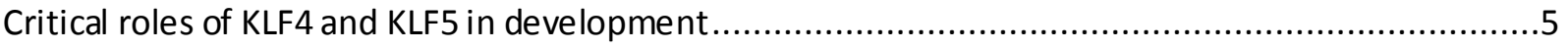

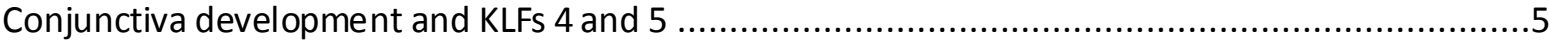

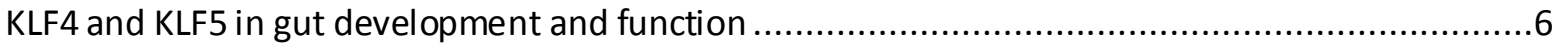

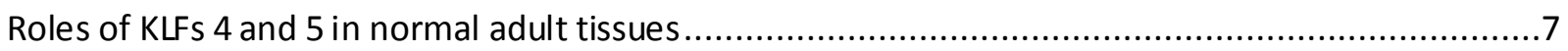

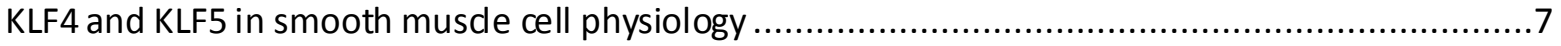

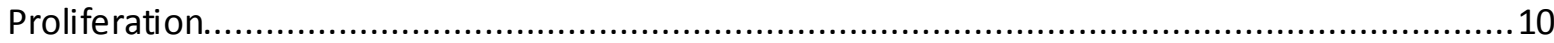

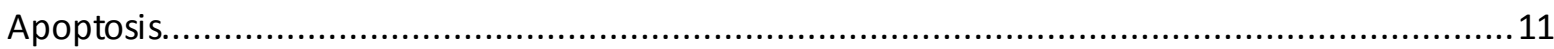

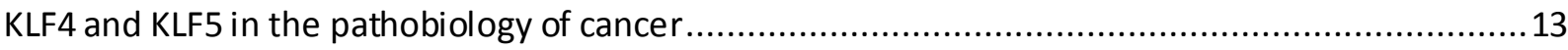

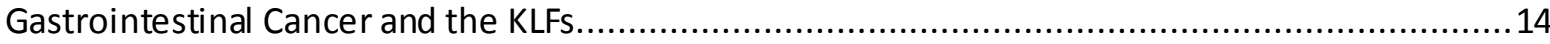

The role of KLFs 4 and 5 in Squamous Cell Carcinoma ....................................................... 16

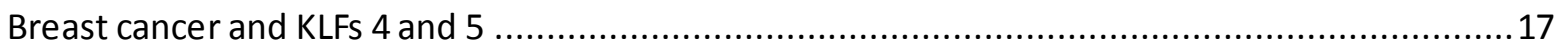

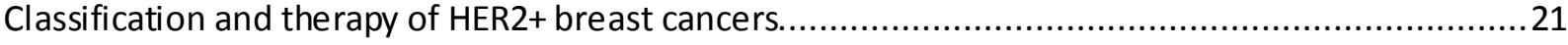

Pathological and molecular classification of breast cancers................................................ 21

Therapeutic strategies and limitations for HER2+ breast cancer ...........................................22

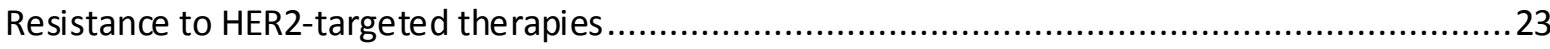

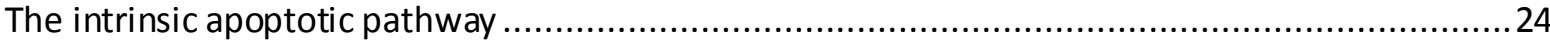

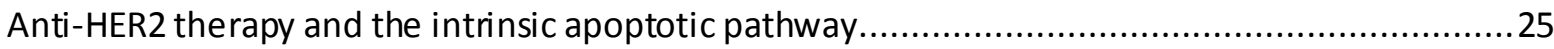

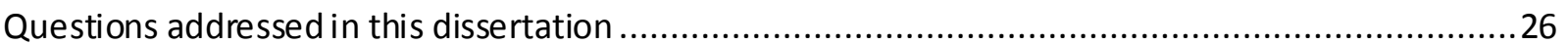

Figure 1. Phylogenetic tree of Kruppel-like factors. ....................................................... 28 


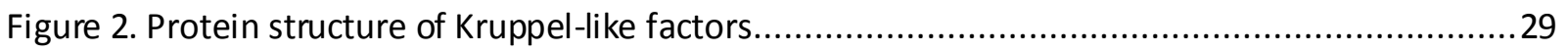

Figure 3. Core-KLF signaling in embryonic stem cells............................................................ 30

Figure 4. Immunostaining of KLF4 and KLF5 in gut epithelium.....................................................31

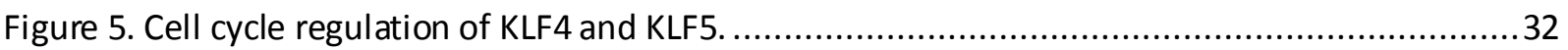

Figure 6. Mechanism of action by trastuzumab..................................................................

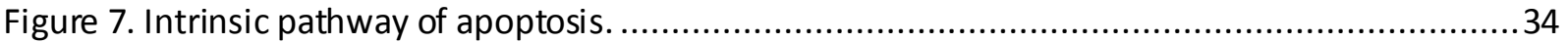

Figure 8. BCL2-family members and their roles in membrane integrity of the Mitochondria, ER and

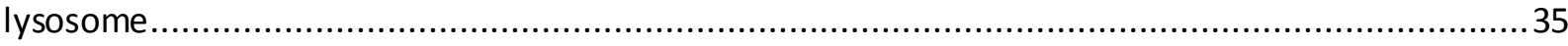

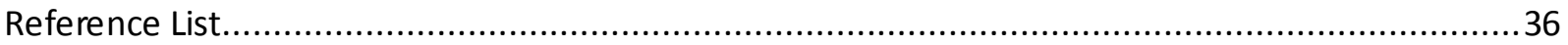

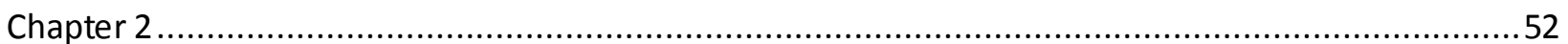

Regulation of anti-apoptotic signaling by Kruppel-like factors 4 and 5 mediates lapatinib resistance in

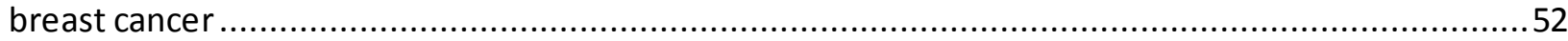

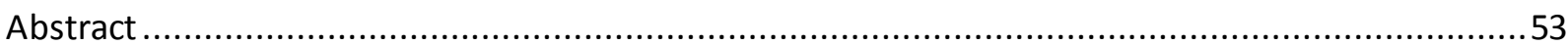

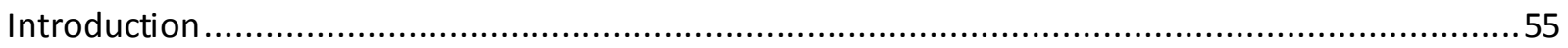

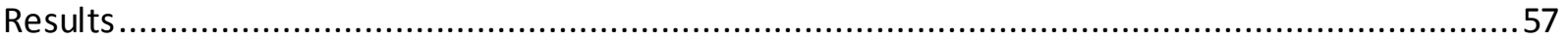

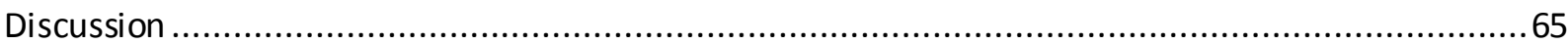

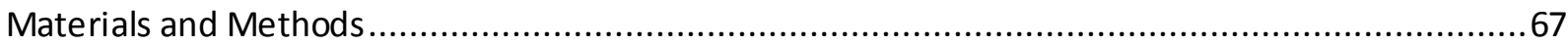

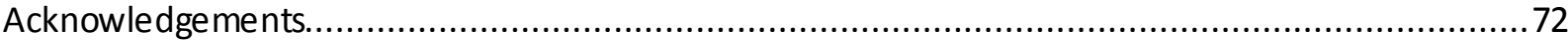

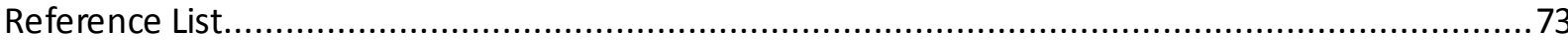

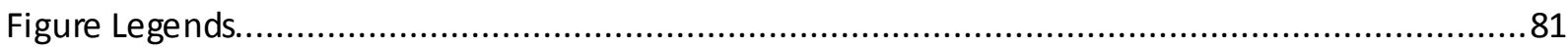

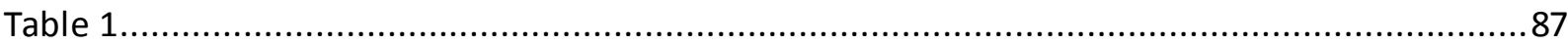

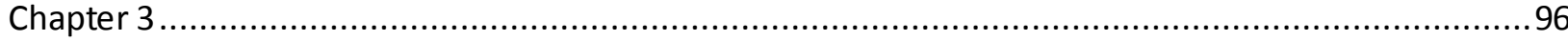

Distinct functions and effector signaling of KLF4 and KLF5 in breast cancer....................................96

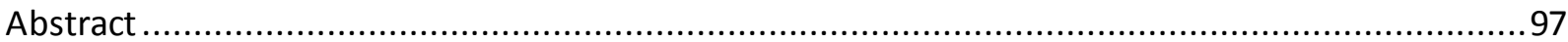

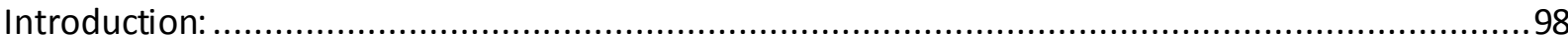

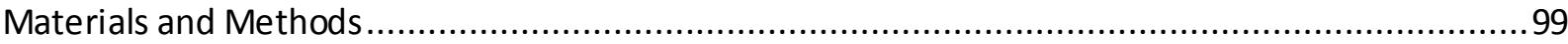

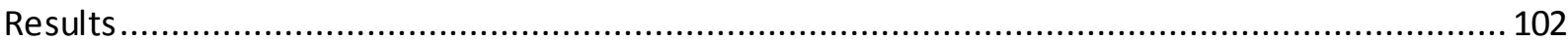

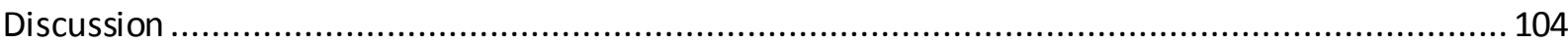

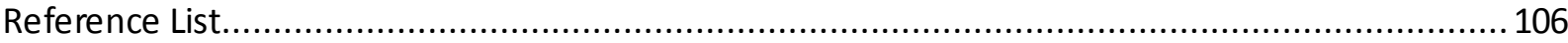

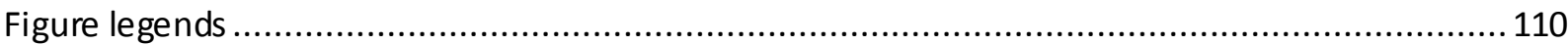

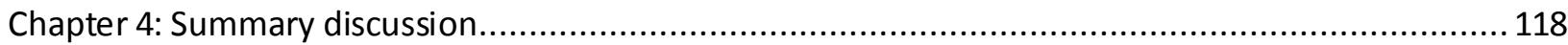

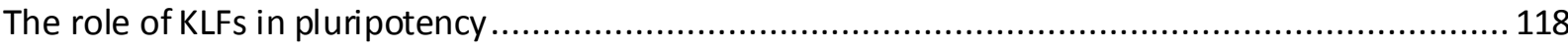


Conserved and distinct functions of KLF4 and 5 in development........................................ 119

Crucial effects of the KLFs in the stress response of the cardiovasculature ............................... 120

Adaptive and survival functions of the KLFs in response to noxious stimuli.................................. 122

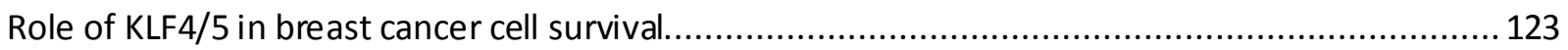

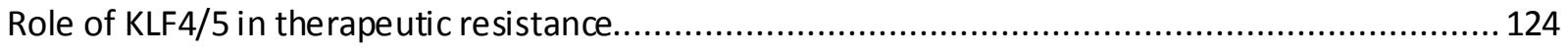

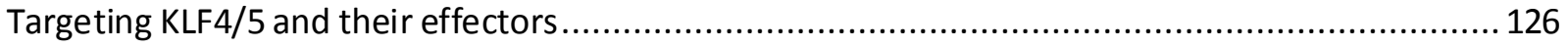

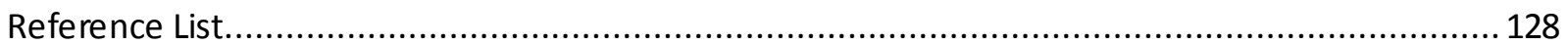

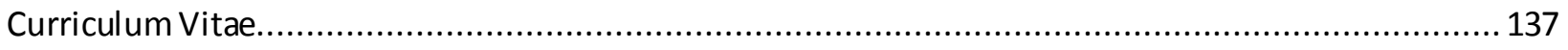




\section{List of Abbreviations}

1-palmytoyl-2-(5-oxovaleroyl)-sn-glycero-3-phosphocholine

POVPC

4',6-diamidino-2-phenylindole

DAPI

Achaete-scute complex homolog 2 (Drosophila)

Ascl2

Adenomatous polyposis coli

APC

All-trans retinoic acid

ATRA

Atonal homolog 1 (Drosophila)

Atoh1

B-Cell lymphoma 2

BCL2

B-Cell lymphoma-extra large

BCL-XL

BCL2 homology domain

$\mathrm{BH}$

BCL2-associated agonist of cell death

Bad

BCL2-associated X protein

Bax

cAMP response binding protein

CBP

Cancer stem cells

CSC

Caudal type homeobox 1

$\mathrm{Cdx} 1$

ChIP-

chromatin immunoprecipitation combined with high throughput sequence

seq

C-Terminal Binding Protein 1

CtBP

Cyclin D1

Ccnd1

Cyclin E

CCNE

Cyclin-dependent kinase inhibitor $1 \mathrm{~A}$

Cdkn1a

Cytokeratin 5

CK5

disease free survival

DFS

Distant metastasis-free survival

DMFS

E74-like factor 3 (ets domain transcription factor, epithelial-specific )

Elf3

Embryonic stem

ES

Epidermal growth factor receptor

EGFR

Epithelial-mesenchymal transition

EMT

Estrogen receptor

ER

Fibroblast growth factor

FGF

Fibroblast growth factor binding protein 1

FGFBP1

Forkhead box A1

Foxa1

Genetically engineered mouse model

GEMM

Hepatocyte nuclear factor 4, alpha

Hnf4a

Histone deacetylase

HDAC

Human epidermal growth factor receptor 2

HER2

Immunohistochemical

IHC

Insulin-like growth factor 1

IGFR1

KLF4 and KLF5

KLF4/5 
Kruppel-like factor

KLF

MicroRNA

$\mathrm{miR}$

MicroRNA-206

miR-206

Mitochondrial membrane integrity

MMI

Mitogen-activated protein kinase

MAPK

Mitogen-activated protein kinase

MAPK

Mouse embryonic fibroblasts

MEF

Mouse mammary tumor virus promoter

MMTV

Mucin 2, oligomeric mucus/gel-forming

Muc2

Myeloid leukemia cell 1

MCL1

Neurogenin 3

Neurog3

Nitric oxide synthase 2, inducible

iNOS

Notch 1 Intracelluar domain

N1ICD

p300/CBP-associated factor

$\mathrm{P} / \mathrm{CAF}$

Peroxisome proliferator-activated receptor $\gamma$

Ppary

Phosphatase and tensin homolog

PTEN

phosphatidylinositide 3-kinase

PI3K

Phosphatidyloinositol-3-kinase

PI3K

Platelet-derived growth factor A

PDGFa

Platelet-derived growth factor receptor

PDGFR

Poly(ADP-ribose) polymerase-1

PARP1

Progesterone receptor

PR

Proliferating cell nuclear antigen

PCNA

qRT-

Quantitative reverse transcription and polymerase chain reaction

PCR

Rat sarcoma viral oncogene homolog

Ras

Receptor tyrosine kinase

RTK

Recombining protein suppressor of hairless

RBP-J

Retinoid acid receptor

RAR

Retinoid X receptor

RXR

Serpin peptidase inhibitor, clade E (nexin, plasminogen activator inhibitor type 1), member 1

PAI-1

Small proline-rich protein

Sprr

Smooth muscle actin

SMA

Smooth muscle cell

SMC

Squamous cell carcinoma

SCC

SRY (sex determining region Y)-box 9

Sox9

C3(1)

SV40 Large T Antigen transgenic mice under control of the C3(1)/prostatein promoter TAg

Tafazzin

TAZ

T-Cell Leukemia/Lymphoma 1

Tcl1 
The Cancer Genome Atlas

TCGA

Transforming growth factor $\beta$

TGF $\beta$

Transgelin 2

$\mathrm{SM} 22 \alpha$

Triple negative breast cancer

TNBC

Tumor necrosis factor $\alpha$

TNF $\alpha$

V-Akt Murine Thymoma Viral Oncogene Homolog 1

Akt1

Vascular endothelial growth factor receptor

VEGFR

v-Myc avian myelocytomatosis viral oncogene homolog

MYC

Wingless-type MMTV integration site family

Wnt

WW Domain Containing E3 Ubiquitin Protein Ligase 1

WWP1 


\section{Chapter 1}

\section{INTRODUCTION AND LITERATURE REVIEW}

\section{KRUPPEL-LIKE FACTORS 4 AND 5}

\section{Kruppel-like factor transcription factors}

Kruppel-like factors (KLFs) are a family of 17 transcription factors involved in diverse biological processes including embryology, development, proliferation, cell fate, cardiovascular disease, and cancer (Fig. 1) ${ }^{1}$. KLFs were named based on their homology to the DNA binding Drosophila melanogaster gap-class segmentation gene "Kruppel”, a critical regulator of thorax and abdomen development ${ }^{2}$. KLFs also share homology to the Sp1 transcription factor, which was one of the first mammalian transcription factors to be identified and described. Sp1, like the KLFs, contains three $\mathrm{C}_{2} \mathrm{H}_{2}$-type zinc finger DNA binding domains that preferentially recognize GC rich regions of $\mathrm{DNA}^{3}$. As KLFs also contain three zinc finger domains, they are classified as part of the Sp1/KLF family. KLFs affect gene transcription via diverse mechanisms which can involve the recruitment of transcriptional coactivators, corepressors as well as additional proteins involved in chromatin remodeling. KLF regulated gene expression is critical for a number of organ systems including cardiovascular, respiratory, digestive, hematological and immune. Furthermore, dysregulation of these factors can be attributed to numerous pathological states such as cancer. KLFs 4 and 5 play major regulatory roles and will serve as the focus of this dissertation. 


\section{Discovery and initial characterization of KLF4 and KLF5}

KLF4 was identified via a low-stringency cDNA library screen using the zinc finger portion of the immediate early transcription factor zif268 and found to be robustly expressed in the colon, thereby being named gut-enriched Kruppel-like factor (GKLF) ${ }^{4}$. KLF5 (BTEB2) was similarly discovered using cDNA library screen of human placenta using rat BTEB cDNA ${ }^{5}$. Subsequent studies found KLF5 to be also highly expressed in the digestive tract, thus also referred to as intestinal-enriched Kruppel-like factor (ILKF) ${ }^{1,6}$. As multiple KLFs were being discovered at this time, their nomenclature was simplified by numbering the Kruppel-like factor, using the date of discovery to dictate the order (IE, KLF4 was the fourth KLF to be discovered) ${ }^{7}$.

Later studies found KLF4 to be expressed prominently in the gut and skin, while expression was detected in various epithelial tissues such as lung, testis, epididymis, cornea, thymus, lymphocyte, and vascular endothelial cells ${ }^{8-14}$. In these tissues, expression of KLF4 was often higher in the terminally differentiated epithelial cells, suggesting a role in differentiation and growth inhibition. Conversely, KLF5 expression appeared to be localized in the intestinal crypts, consistent with a role in proliferation ${ }^{15}$.

Human KLF4 is located on chromosome 9q31 and spans a 6.3kb region. The processed transcript is $3.5 \mathrm{~kb}$, composed of 5 exons ${ }^{16}$. The coding region produces a 470 amino acid protein, with a predicted molecular mass of $\sim 59 \mathrm{kDa}$. As with all KLFs, the three zinc finger motifs are found at the carboxy-terminus end of the molecule (Fig. 2). Additional functional domains are a transcriptional activation domain located at the amino-terminus, a repression domain located within the DNA binding domain at the carboxy terminus as well as a nuclear localization signal found immediately amino terminal to the DNA binding domain ${ }^{16}$. Human KLF5 is located at 
$13 q 22.1$ and spans $22.57 \mathrm{~kb}$ region. KLF5 mRNA is $3.5 \mathrm{~kb}$, composed of 4 exons. KLF5 is 457 amino acids long with a predicted molecular mass of $\sim 57 \mathrm{kDa}^{5}$.

\section{Protein Structure of Kruppel-like factors}

The structure of the KLFs is highly conserved among mammals, and additionally KLFhomologs can be found even as far as $C$. Elegans. The three $\mathrm{C}_{2} \mathrm{H}_{2}$ zinc finger motifs common to all KLFs consists of zinc tetrahedrally coordinated by cysteine and histidine residues, allowing the domain to fold into a $\beta \beta \alpha$ structure $^{1}$. KLFs have three highly conserved zinc finger motifs at the carboxy-terminus ends of the proteins (Fig. 2). The first and second of these zinc fingers are comprised of 25 amino acids, while the third is composed of $23^{1}$. Each zinc finger recognizes a three base pair sequences, totaling 9 base pairs for each KLF. The DNA binding sites are similar for KLFs, recognizing GC rich sequences with a core motif of 5'-CACCC- ${ }^{\text {, }}{ }^{1}$. Additionally, the DNA binding domain of KLFs can also be important for nuclear localization for certain members of the family ${ }^{16}$.

Although the carboxy-terminal DNA binding domains are highly conserved among the KLFs, the amino-terminal regions can vary significantly. Variability in the amino-terminal regions allows the KLFs to bind numerous different coactivators, corepressors, and modifiers. The ability to bind and recruit diverse cofactors allows the KLFs to elicit distinct and unique functions in numerous organ systems, despite having near identical DNA binding domains. Some of these cofactors include cAMP response binding protein (CBP), p300, p300/CBP-associated factor (P/CAF), CtBP and SIN3A ${ }^{1}$. Additionally, KLF activity can be altered by posttranslational modifications. KLF5 contains sites for post-translational modifications such as a phosphorylation site at Ser153, acetylation site at Lys369 and a region 324-328 predicted to 
interact with the ubiquitin-ligase WWP1 $1^{17,18}$. KLF4 has also shown to be acetylated and the acetylation of the KLFs generally enhances their transcriptional activity ${ }^{1}$. Other posttranslational modifications such as phosphorylation, SUMOylation, and ubiquitination can either enhance or suppress translational activity of certain KLF family members depending on the cellular context ${ }^{1}$. Through this, KLFs can demonstrate numerous distinct roles and functions in various physiological and pathophysiological processes.

\section{Function of KLFs 4 and 5 in pluripotent stem cells}

KLF4 is one of the four Yamanaka transcription factors required for induced pluripotency ${ }^{19}$. When KLF4 is coexpressed with MYC, Oct3/4 and Sox2 in adult mouse fibroblasts, the cells dedifferentiate into pluripotent stem cells. In addition, KLF4 has functions in the native embryonic stem cell population as well. KLFs 2, 4 and 5 are part of a "core KLF" circuitry that redundantly maintain the stem cell phenotype in mouse embryonic stem (ES) cells $^{20}$. To induce differentiation, all three KLFs must be depleted. Any one factor is suitable for the maintenance of ES characteristics. This observed relationship is attributed to the "core KLFs" ability to act as an enhancer for Nanog, a prominent transcription factor in ES cell biology (Fig 3). By maintaining Nanog levels, the "core KLFs" preserve a positive feedback loop which keeps the cell in the ES state. Reduction of all three factors causes Nanog levels to drop, this breaking the positive feedback signaling and resulting in differentiation. Although the "core KLFs" demonstrated redundant roles in this setting, alternative modalities to augment individual KLF

expression, such as genetic deletion, can induce differentiation ${ }^{21}$. In agreement, there is evidence that KLF5 can exhibit unique functions in ES cells ${ }^{22}$. 


\section{Critical roles of KLF4 and KLF5 in development}

Genetic deletion studies have found that homozygous ablation of KLF4 or KLF5 is perinatally or prenatally fatal, respectively. $K I f 4^{-/-}$mice are able to survive birth but die shortly thereafter ${ }^{23}$. This is likely due to a barrier defect in the skin that results in massive water loss and death. As KLF4 is robustly expressed in the differentiating layers of the epidermis, the Klf4 homozygous knockout may prevent proper epithelial maturation ${ }^{24}$. Indeed, overexpression of KLF4 in the skin has been shown to accelerate terminal differentiation and the formation of the permeability barrier ${ }^{25}$. This relationship has been attributed to the upregulation of Sprr and keratin gene families that are critical for epithelial barrier integrity.

$K_{I f 5^{-/-}}$mice die before E8.5 due to a defect in embryo implantation ${ }^{26-28}$. Ablation of Klf5 results in premature differentiation, attributed to a loss of KLF5 mediated phosphorylation of Akt1 by Tcl1. Heterozygous mice for Klf5 are viable and fertile, however they demonstrate defects in response to injury, angiogenesis and adipocyte differentiation ${ }^{29,30}$. Ectopic expression of KLF5 in epidermal tissues disrupts epithelial-mesenchymal interactions required for proper skin and craniofacial development ${ }^{31}$. Additionally, forced expression of KLF5 elicits defects in the regenerative potential of keratinocytes, suggesting that KLF5 may play a role in epithelial turnover.

Conjunctiva development and KLFs 4 and 5

Tissues specific knockout studies have elucidated important roles for the KLFs in proper development of the conjunctiva, the epithelial barrier that protects the eye. Selective deletion of Klf4 from the surface ectoderm derived structures of the eye (cornea, lens, conjunctiva and 
eyelids) results in swollen, vacuolated basal and epithelial cells with edematous stroma ${ }^{32}$.These conjunctiva lacked goblet cells and the anterior cortical lens also displayed a vacuolated morphology. Conditional knockout of Klf5 in surface ectoderm derived structures of the eyes also revealed critical functions for KLF5 in the postnatal maturation of these tissues. Ablation of Klf5 results in hyperplastic keratinocytes, enlarged hair follicles and sebaceous glands, and malformed meibomian glands ${ }^{33}$. In addition to corneal and lacrimal defects, these mice also displayed a lack of goblet cells similar to Klf4 deletion, suggesting that both Klfs are critical for goblet cell formation.

Furthermore, these investigators coablated both Klf4 and Klf5 in these tissues. The resulting phenotype was far more severe than individual deletions, suggesting that these factors may be complementing, not redundant in this setting.

\section{KLF4 and KLF5 in gut development and function}

In addition to the conjunctiva, KLF4/5 have critical roles in the development and function of gut epithelium. Normally, KLF4/5 exhibit differential expression patterns in the mature gut as visualized by immunostaining, with KLF4 preferentially staining the post-mitotic, differentiated villi tips and KLF5 localized to the proliferative basal crypts (Fig 4) ${ }^{24}$. Despite this, genetic deletion studies elicited shared roles in goblet cell formation in the gut, consistent with their roles in conjunctiva formation. Conditional $\mathrm{Klf}^{-/-}$mice demonstrate normal proliferation, cell death and proper colonocyte and enteroendocrine cell formation. However, these mice lack up to $90 \%$ of colonic goblet cells comparative to wild type control ${ }^{34}$. Furthermore, by in situ hybridization, the gut of conditional $\mathrm{Klf}^{-/}{ }^{-}$mice have reduced expression of Muc2, a goblet cell specific marker. The few goblet cells these mice do have display an altered morphology, lacking 
secretory vesicle formation. Additionally, KLF5 functions to promote proper gut maturation and function. The gut of conditionally deleted Klf5 mice lacked normal brush border formation of enterocytes and exhibited a loss of enteroendocrine and goblet cells ${ }^{35}$. While the proliferation of the gut in these mice appeared unaffected, the expression of several epithelial differentiation genes was reduced such as Elf3, Ppary, Atoh1, Ascl2, Neurog3, Hnf4a, and Cdx1. Furthermore, Klf5 deletion inhibited the normal downregulation of Foxa1 and Sox9 that accompanies proper villi morphogenesis. Similar to the functions in conjunctiva development, KLF4/5 contribute to development of the gut epithelium. In this setting, KLF4/5 impact goblet cell formation, albeit in two different sections of the gut, colon and small intestine respectively. These observations taken together with their roles in the conjunctiva suggest that KLF4 and KLF5 are critical transcription factors for the generation of goblet cells and normal epithelial function.

\section{Roles of KLFs 4 and 5 in nomal adult tissues}

\section{KLF4 and KLF5 in smooth muscle cell physiology}

KLF4/5 exhibit shared and unique roles in development, however studies have also demonstrated vital roles for these factors in adult tissues. The smooth muscle cells (SMCs) of the vasculature have been a particular area of interest with regards to the function of these KLFs. The expression of KLF4/5 in SMCs is regulated both in development as well in various biological processes. For example, KLF5 activates the expression of smooth muscle myosin heavy chain by binding to a targeted sequence in the respective promoter region of the gene ${ }^{36}$. Yet, robust expression of KLF5 is only observed in fetal aortic SMCs and is lost in adults, implicating a specific role for KLF5 in cardiovascular development ${ }^{36,37}$. However, the reexpression of KLF5 has been observed in adult SMCs under pathological conditions such as 
coronary atherosclerosis, vein graft hyperplasia, and response to injury ${ }^{37-40}$. Neointimal expression of KLF5 is induced following aortic balloon injury, furthermore KLF5 is expressed in most human coronary lesion samples ${ }^{37}$. In fact, KLF5 expression is correlated with the incidence of restenosis, and functionally KLF5 promotes coronary atherectomy samples outgrowth in culture, a characteristic associated with a higher likelihood of restenosis ${ }^{41}$. This association with proliferation of SMCs and KLF5 is seen in other contexts as well. Various stimuli (Angiotensin II, TNF- $\alpha$, survivin and MAPKs) that promote the growth of SMCs also induce KLF5 expression $^{41}$. Overexpression of KLF5 in these cells increases vascular injury response factors such as iNOS, PAI-1, PDGF-A and VEGFRs ${ }^{17,38,42,43}$. These results suggest KLF5 may mediate SMCs proliferative response to injury.

Genetic studies in mice further elaborated on the role of KLF5 in cardiovascular pathophysiology. Mice heterozygous for Klf5 displayed thinner medial and advential layers of the aorta comparative to wild type mice ${ }^{26}$. Additionally, these mice had a reduced response in the cuff-induced injury assay ${ }^{26}$. The femoral arteries of $\mathrm{KlF}^{+/-}$mice showed reduced activation and growth of SMCs, inflammatory response and angiogenesis. Such defects ultimately result in smaller areas of neointimas and a reduction in granulation tissues. Am80, a retinioid based molecule that attenuates KLF5's transcriptional activity produces similar deficits as heterozygous knockout ${ }^{26}$.

Mechanistically, KLF5 facilitates the proliferation of SMCs. Angiotensin II promotes the growth of SMCs and stimulation of SMCs with angiotensin II upregulates KLF5 mRNA and protein $^{38,44}$. Furthermore, SMC treatment with angiotensin II stimulates the phosphorylation of KLF5, thereby increasing its association with c-Jun and ultimately repressing cell cycle inhibitor 
p21 ${ }^{\text {Waf1/Cip1 }}$. MAPK signaling is believed to mediate KLF5 phosphorylation, as small molecule inhibition of MEK abrogates angiotensin II induced phosphorylation of KLF5 ${ }^{45}$.

KLF4 also has significant roles in SMC physiology and function. Similar to KLF5, KLF4 levels are normally low in the vasculature but they are induced in the medial layer upon injury ${ }^{46-}$ ${ }^{48}$. In the vasculature, injury typically results in repression of several differentiation markers in $\mathrm{SMCs}^{48}$. In mice with a conditional knockout for Klf4, the suppression of these markers is transiently delayed ${ }^{48}$. Additionally, conditional $\mathrm{Klf4}^{-/-}$mice undergo a hyperproliferative response to injury in the neointima comparative to wild type control. These results suggest that Klf4 faciliates dedifferention and inhibition of proliferation in SMCs following injury. KLF4 expression is also induced by oxidized phospholipids such as 1-palmytoyl-2-(5-oxovaleroyl)-snglycero-3-phosphocholine (POVPC) that are known to accumulate in atherosclerotic lesions ${ }^{49}$. While POVPC suppresses the expression of SMC differentiation markers including $\alpha$-SMA and SM heavy chain, it increases the levels of inflammatory associated mediators such as MCP-1 and TGF- $\beta^{49}$. It believed that KLF4, along with Elk-1 and HDACs, mediate the effect POVPC has on SMC differentiation markers ${ }^{47}$. Furthermore, KLF4 expression is required for POVPC mediated stimulation of migration, as well as POVPC induced expression of several extracellular matrix proteins $^{50}$. Therefore, it is possible that KLF4 plays a major role in atherosclerotic disease of the vasculature.

Interestingly, although KLF5 promotes SMC proliferation, KLF4 appears to inhibit SMC growth. Overexpression of KLF4 in SMCs inhibits proliferation via a p53-dependent process ${ }^{51}$. Furthermore, All-trans retinioic acid (ATRA) upregulates KLF4 and is required for ATRAmediated inhibition of SMC growth ${ }^{52}$. KLF4 is believed to inhibit SMC growth by repressing several known promoters of cellular growth such as platelet-derived growth factor receptor 
(PDFGR)- $\beta{ }^{53}$. KLF4 appears to have pleiotropic effects in the regulation of smooth muscle associated genes. Although KLF4 induces SM22 $\alpha$ and $\alpha$-SMA expression in response to ATRA treatment, incubation of SMCs with TGF- $\beta$ and PDGF-BB results in KLF4-mediated SM22 $\alpha$ and $\alpha-S M A$ repression $^{46,53,54}$.

Even though KLF4 and KLF5 appear to have opposing functions on SMC growth, both are simultaneously upregulated in response to vascular injury. It is possible that although these functions seem antagonistic, the combination of KLF4 and KLF5 allow cells to tightly regulate proliferative and dedifferentiating influences, thus ensuring proper stress response. A possible candidate that coregulates KLF4 and KLF5 expression in response to injury is microRNA (miR) 145. miR-145 is highly expressed in the normal vasculature and downregulated in vessel walls upon neointimal lesion formation. miR-145 also been shown to regulate SMC cell fate and directly targets both KLF4 and KLF5 ${ }^{55,56}$. Therefore, control of miR-145 expression may be part of a critical switch in the injury response program of SMCs via regulation of KLF4 and KLF5.

\section{Proliferation}

The most widely characterized functions of KLF4 and KLF5 involve their effects on proliferation. Across multiple different tissues, KLF4 tends to be an inhibitor of proliferation while KLF5 promotes cellular growth (Fig. 5) ${ }^{57-59}$. In agreement, KLF4 expression is often localized to the post-mitotic, well differentiated compartments of epithelia while KLF5 expression is concentrated in the proliferative, transit amplifying compartments (Fig. 4) ${ }^{24}$.

Initially, it was found that KLF4 is lowly expressed during active proliferation and, conversely, is upregulated upon serum starvation or contact inhibition ${ }^{4}$. Further studies utilized 
cellular response to $\gamma$-irradiation to mechanistically characterize KLF4's role in cell cycle and growth. Upon $\gamma$-irradiation exposure, KLF4 levels are increased in a p53-dependent manner. KLF4 binds to the promoter for $p 21^{\text {Waf1/Cip1 }}$ and actively recruits $\mathrm{p} 53$ to this region, thus facilitating the expression of this cell cycle inhibitor ${ }^{60,61}$. In addition to promoting cell cycle inhibitors, KLF4 suppresses the expression of Cyclin B1, Cyclin D1 and Cyclin E1 ${ }^{57,60,62}$. In particular, by suppressing Cyclin E1, KLF4 was found to maintain chromosomal integrity during $\gamma$-irradiation treatment ${ }^{61}$. Based on these functions, KLF4 is considered to be an inhibitor of cell growth that prevents inappropriate cell cycle progression and preserves chromosomal stability.

KLF5 promotes proliferation in various tissues and cellular contexts. In contrast with KLF4, KLF5 levels are downregulated during serum starvation or contact-inhibition mediated quiescence $^{15}$. Additionally, KLF5 participates in several mitogenic signaling pathways, such as RAS/MAPK, phosphatidyloinositol-3-kinase (PI3K) and protein kinase $C^{57,63,64}$. Furthermore, KLF5 transcriptionally targets several genes known to promote cell cycle progression such as Cyclin D1, Cyclin B1, and Cdc2. Moreover, KLF5 represses the expression of the cell cycle inhibitory proteins p15 and p27 $7^{65}$. Interestingly, although conditional genetic deletions of Klf4 or Klf5 result in similar deficits (IE, loss of goblet cells), functional studies suggest the two elicit opposing influences of cell growth and cell cycle progression.

\section{Apoptosis}

The inhibitory effect of KLF4 on cell cycle supports its anti-apoptotic functions. Upon DNA damage, cell must undergo growth arrest and activate repair mechanisms, or else commit to apoptosis. As KLF4 inhibits the cell cycle, it allows cells to recover from DNA damaging insults during the growth arrest phase. If these cells were to undergo cell division with DNA 
damage, they would likely undergo apoptosis. For example, overexpression of KLF4 in RKO colon cancer cells reduces the number of apoptotic cells following $\gamma$-irradiation exposure ${ }^{66}$. In addition to slowing proliferation to protect against apoptosis, KLF4 can directly downregulate pro-apoptotic machinery. In this setting, KLF4 blocked the upregulation of the pro-apoptotic protein BAX via inhibiting the p53-mediated transactivation of the $B A X$ gene ${ }^{67}$. In fact, KLF4 frequently interacts with either the p53 protein or promoter. For example, in MDA-MB-134 breast cancer cells KLF4 has been shown to bind and suppress p53 transcription. Furthermore, the suppression of p53 by KLF4 is critical for bypassing RAS-inducing senescence in mouse embryonic fibroblasts ${ }^{68}$. These results highlight the possible importance of KLF4 in postmitotic, differentiated epithelium, conveying both durability and cell cycle arrest.

KLF5 is also believed to suppress apoptosis. Ectopic expression of KLF5 in EU-8 leukemia cells stimulates the expression of survivin, a well-documented inhibitor of apoptosis. Furthermore, depletion of KLF5 reduces survivin expression, ultimately sensitizing these cells to chemotherapy induced apoptosis ${ }^{69}$. In these cells, KLF5 binds directly to p53, thus blocking the p53-mediated suppression of survivin expression. Additionally, KLF5 has been shown to be upregulated in response to 5-fluorouracil treatment and ultraviolet irradiation of colon cancer cells ${ }^{70}$. Depletion of KLF5 and blocking this induction sensitizes these cells to apoptosis in response to DNA damaging agents. Unlike KLF4, the activity of KLF5 was not dependent on p53 activity ${ }^{70}$. Instead, the prosurvival effects of KLF5 are attributed to its association with the kinase Pim1, a negative regulator of the pro-apoptotic protein BAD. Additional contexts reveal that KLF5 can also promote survival in SMCs via interacting with poly(ADP-ribose) polymerase-1 (PARP1) ${ }^{71}$. 


\section{KLF4 and KLF5 in the pathobiology of cancer}

KLF5 is considered as an oncogene in a variety of cancers, including intestinal, gastric and breast cancer ${ }^{59,64,72,73}$. KLF5 is generally regarded to enhance the cancer process, often by promoting proliferative signaling. However, the influence of KLF4 in cancer has been characterized as context-dependent ${ }^{68}$. This definition was generated from the observed relationship between KLF4 in RAS-induced senescence of mouse embryonic fibroblasts (MEFs). In this setting, ectopic KLF4 was sufficient to rescue senescence as a result expression of activated RAS. KLF4 achieved this effect by suppression of endogenous Tp53, allowing the cells to re-enter the cell cycle. In this role, KLF4 promotes tumor progression and allows for robust transformation of MEFs. However, without the presence of activated RAS in the cells, ectopic KLF4 rapidly induces cell cycle arrest, likely due to the upregulation of the cell cycle inhibitor Cdkn1a. Thus, KLF4's activity is described as context-dependent. In a setting which neutralizes the growth suppressive effect of KLF4, for example by upregulation of Ccnd1 via activated RAS, KLF4 enhances malignant properties. However, if the anti-proliferative signaling of KLF4 is maintained, then the overall influence of its expression is likely to be tumor suppressive. In agreement, studies have reported both oncogenic and tumor suppressive roles of KLF4. It is important to interpret individual studies according to the methodologic approach. For example, studies that utilize primarily exogenous KLF4 (enforced expression) may not accurately or consistently reflect the function of the endogenous factor. 


\section{Gastrointestinal Cancer and the KLFs}

KLFs 4 and 5 are robustly expressed in the gastrointestinal(GI) tract and both are critical for proper GI development. KLF4/5 are differentially expressed in gut epithelium and functional studies suggest that KLF4/5 elicit different functions in neoplasms arising for GI-associated structures, consistent with a tumor suppressor role for KLF4 and KLF5 as an oncogene. KLF4 mRNA and protein expression is downregulated in colon cancers ${ }^{74}$. Additionally, KLF4 expression is inversely correlated to the size of tumors in the well-established Adenomatous polyposis coli $(\mathrm{Apc})^{\mathrm{min} /+}$ genetically engineered mouse model of colon cancer ${ }^{75}$. Furthermore, haploinsufficiency of Klf4 in this model increases adenoma formation in mice. Apc is a critical regulator of Wnt signaling and is believed to help maintain epithelial homeostasis via control of cellular levels and location $\beta$-catenin ${ }^{76-78}$. KLF4 has been shown to be regulated by Apc and both ultimately function to inhibit Wnt signaling and tumorigenesis in colon cancer ${ }^{79,80}$. KLF4 appears to accomplish this by suppression of $\beta$-catenin transcription, direct interactions with $\beta$ catenin and repression of $\beta$-catenin/TCF4 transcriptional activity. In addition to suppressing Wnt signaling, KLF4 exerts growth suppressive effects on colon cancer cells in culture. KLF4 expression is markedly reduced in RKO colon cancer cells and reintroduction of KLF4 in this setting upregulates $p 21^{\text {Waf1/Cip } 1}$ expression resulting in G1/S cell cycle arrest ${ }^{81}$. Furthermore, ectopic expression of KLF4 in these cells reduces colony formation, cell migration, invasion and tumor formation in mice. Therefore, KLF4 may inhibit colon cancer formation and progression via punitive effects on Wnt activity and proliferative signaling.

On the other hand, KLF4 can exert protumorigenic signaling within the cancer stem-like subset of colorectal cancer cells, and promotes cell survival in irradiated tumor cells ${ }^{82}$. Consequently, the description of KLF4 as either an oncogene or a tumor suppressor gene may be 
less informative relative to its consideration as a stress responsive regulator of cell fate that can have pleiotropic effects in cancer. This is particularly true since genetic changes within KLF4 are rare in cancer, including colorectal cancer.

KLF5 more consistently promotes oncogenesis in GI-associated tissues. Consistent with its expression in the basal compartment, KLF5 promotes proliferation in colon cancer cells ${ }^{83}$. Additionally, KLF5 has been shown to be critical in RAS signaling, which frequently is found to have activating mutations in colon cancer. KLF5 has been reported to mediate the transformation of NIH3T3 cells by activated HRAS or KRAS ${ }^{63,84}$. Furthermore, KLF5 expression has been shown to be increased in both primary human tumors and genetically engineered mouse models of colon cancer that harbor activating KRAS mutations ${ }^{85}$. Comparative to Klf5 wild type, $\mathrm{Apc}^{\mathrm{min} /+}$ mice heterozygous for Klf5 exhibit a 96\% reduction in adenoma formation. The reduction of precancerous lesions in these mice correlates with decreased nuclear staining of $\beta$ catenin and diminished levels of its putative target genes, CCND1 and c-Myc. KLF5 has been shown to facilitate the nuclear localization of $\beta$-catenin, as well as promote the transcriptional activation of this pathway in vitro ${ }^{86}$. Moreover, an inducible deletion model has elicited vital roles for Klf5 for maintaining the integrity and oncogenecity of colon cancer stem cells ${ }^{87}$. Therefore, KLF5 may mediate the activation of Wnt signaling that is required for colon cancer formation and progression.

In addition to colon cancer, KLF5 has roles in neoplasms of other gastrointestinal tissues. KLF5 expression is correlated with small early stage, localized gastric cancers ${ }^{88}$. Helicobacter Pylori is a major risk factor for human gastric adenocarcinoma. Helicobacter Pylori infection of gastric epithelial cells induces KLF5 expression via the virulent factors of the pathogen, including the cag pathogenicity island and the vacuolating cytotoxin ${ }^{73}$. Consistent with an 
oncogenic role of KLF5 in gastric cancer, expression of KLF5 is elevated in dysplastic and metaplastic lesions of the stomach comparative to normal tissue.

The role of KLFs 4 and 5 in Squamous Cell Carcinoma

Several studies have evaluated the role of KLF in squamous cell carcinomas (SCC), particularly in the skin. Expression analysis found KLF4 to be highly expressed in SCCs of the skin and larynx ${ }^{89}$. While KLF4 expression is normally localized to differentiated, post-mitotic compartment of the cutaneous and oral epithelium, expression in other compartments is attributed to pathogenesis. Conditional forced expression of KLF4 in the basal layers of the skin in mice resulted in dysplasia and the formation of SCC-like lesions ${ }^{90}$. Interestingly, as a proposed mechanism of KLF4-induced transformation requires neutralization of its growth-suppressive effects, in this context both KLF4 and PCNA protein were coexpressed, suggesting these cells were proliferating despite robust KLF4 expression ${ }^{91}$. Additional work focusing on KLF4mediated SCC formation revealed a strong upregulation of several retinoic acid receptors, including retinoid acid receptor- $\gamma(\mathrm{RAR}-\gamma)$ and retinoid X receptor- $\alpha(\mathrm{RXR}-\alpha)^{92}$. Although induced by KLF4, stimulation of these receptors antagonized KLF4 activity. Therefore, it was found that treating mice with KLF4-induced SCC with a selective RXR agonist prevents the formation of cutaneous tumors. In contrast, disruption of epithelial maturation in the oral cavity by homozygous ablation of Klf4 results in dysplastic lesions, increased proliferation and an abnormal differentiation ${ }^{93}$. Furthermore, introducing activated KRAS into these mice results in rapid SCC formation in the tongue.

On a cautionary note, as disruption of the epithelial permeability barrier can elicit protumorigenic inflammatory responses that promote tumorigenesis in a non-cell autonomous 
fashion, the homozygous knockout of KLF4 across larger areas of the skin or oral mucosa may not provide an accurate model of spontaneous carcinogenesis and clonal evolution ${ }^{94}$.

KLF5 expression has been found to positively correlated with lymph node metastasis and local reoccurrence in SCC of the larynn ${ }^{95}$. Although KLF5 expression was also correlated with several markers of epithelial-mesenchymal transition (EMT), at this point functional evidence supporting a role in SCC is insufficient.

Breast cancer and KLFs 4 and 5

Although KLF4 and KLF5 have been implicated in malignancy of other tissues, their role in breast cancer has been of particular interest and has been extensively detailed in the literature. While generally KLF5 promotes breast malignancy, KLF4 appears to act as either an oncogene or tumor suppressor depending on the setting.

KLF4 was first characterized as an oncogene by its ability to transform immortalized rat kidney epithelial cells (RK3E) ${ }^{89}$. cDNA libraries were constructed based off of human SCC and breast cancer cell lines to identify genes involved in cellular transformation. Expression cloning revealed KLF4 (GKLF) and c-MYC to be capable of inducing 2D foci formation in RK3E cells. Additional studies found that KLF4 is upregulated in a variety of cancers, including $70 \%$ of breast cancers as determined by immunostaining or in situ hybridization, compared to uninvolved normal breast ${ }^{96}$. Immunohistochemical (IHC) analysis of breast cancer samples and patient outcome found nuclear localization of KLF4 in small breast tumors to be clinically aggressive $^{97}$. Furthermore, 59\% of canine breast carcinomas demonstrate higher than normal IHC staining of KLF4 ${ }^{98,99}$. Additionally, in this study strong nuclear expression of KLF4 was 
associated with shorter survival when compared to low/intermediate levels. Methylation studies found KLF4 to be preferentially demethylated in clinically aggressive tumors, such as basal-like breast cancer ${ }^{100}$. Moreover, demethylation of KLF4 was correlated with poor patient outcome.

While these observations are consistent with an oncogenic role for KLF4 in breast cancer, other studies differ in their findings. Using the publically available microarray database Oncomine, KLF4 was found to be downregulated in tumors comparative to normal breast ${ }^{101}$. Furthermore, KLF4 transcripts are inversely correlated with histological grade. In addition, KLF4 levels are elevated in $\mathrm{ER}^{+}$tumors, which generally have a more favorable prognosis. In agreement with these observations, an independent microarray database found elevated KLF4 to forecast prolonged disease-free survival in breast cancer patients, particularly in patients with $\mathrm{ER}^{+}$tumors ${ }^{102}$. Additionally, comparative to certain non-transformed breast epithelial cells such as MCF10A, the expression of KLF4 is decreased in several breast cancer cell lines ${ }^{103}$.

Similar to the conflicting reports on the expression of KLF4 in breast cancer, functional analysis indicates both oncogenic and tumor suppressor roles for the transcription factor. Forced expression facilitates RK3E foci formation through non-canonical Notch1 signaling ${ }^{104}$. KLF4 was observed to directly bind the Notch1 promoter in immortalized breast epithelial cells, MCF10a, indicating a possible effector pathway in breast cancer. Given the importance of KLF4 in pluripotency, KLF4 has been found to impact cancer stem cell characteristics in breast cancer. Reduction of KLF4 suppressed the mammosphere forming efficiency in breast cancer cells, an in vitro assay that enriches for the cancer stem cell (CSC) population ${ }^{105}$. Furthermore, KLF4 depletion elicited defects in migration, invasion, and tumor formation, suggesting a protumorigenic role for KLF4 in the breast ${ }^{105,106}$. Moreover, KLF4 has been shown to promote metastasis formation in the lungs via recruitment of myeloid-derived suppressor cells ${ }^{107}$. 
Although the mechanisms of these effects are currently unclear, other studies have linked KLF4 to specific effector pathways. KLF4 has been shown to suppress p53 and to upregulate mircroRNA-206 (miR-206), a recently characterized oncogenic microRNA in breast cancer ${ }^{106}$. Furthermore, KLF4 is induced by progesterone signaling and implicated in the progesteronemediated expansion of the CSC population in human luminal breast cancer cells ${ }^{108}$. Consistent with a role for KLF4 in hormone signaling, reduction of KLF4 impedes the mitogenic effects of estrogen stimulation in breast cancer ${ }^{109}$.

In contrast, other studies report tumor suppressor activities for KLF4 in breast malignancy. Manipulations of exogenous and endogenous KLF4 in breast cancer, as well as other cellular contexts, demonstrate an inhibitory role towards on cell growth ${ }^{58,106}$. Moreover, exogenous KLF4 can repress metastasis formation in xenografted mice ${ }^{102,103}$. Additional work found that KLF4 directly binds and regulates multiple genes involved in EMT such as Ecadherin, promoting an epithelial phenotype ${ }^{58,102}$. Although human metastatic tumors retain the epithelial characteristics of the primary lesion, mesenchymal characteristics are often associated with malignant properties such as migration, and epithelial features can be associated with reduced migration, invasion, and tumor formation. Furthermore, despite KLF4 being required for the growth promotion of ER signaling in breast cancer, additional evidence suggests an inhibitory role for KLF4 in the ER pathway ${ }^{101}$.

Taken together, these paradoxical observations on the function of KLF4 in breast cancer make it difficult to ascertain the overall influence of the gene in this disease. The apparent context-dependent role of KLF4 in breast cancer highlights the importance of both the model system and experimental approach in which its functions are tested. Given the absence of clear genetic alterations pointing to a critical function of KLF4 during tumorigenesis, these disparate 
results are perhaps unsurprising. Additional work is needed to clarify which in vitro contexts most accurately reflect in vivo conditions in hopes of gaining a better understanding of the role of KLF4 in breast malignancy. Despite its unclear or complex role in cancer pathogenesis, the studies described below implicate KLF4 as an active component of the breast cancer drug resistant phenotype, and point to specific therapeutic opportunities to overcome drug resistance.

Although KLF5 was initially observed to exhibit some degree of deletion in up to $70 \%$ of breast cancer cell lines, subsequent studies have generally reported oncogenic functions for $\mathrm{KLF}^{110}$. Consistent with its expression in basal epithelia, KLF5 is linked to proliferative breast cancers. Analysis of mRNA in primary human cancers associated KLF5 transcripts with highly aggressive tumors with elevated mitotic indices such as basal-like and HER2+ breast cancer ${ }^{72}$. Additionally, promoter methylation of KLF5 is also associated with poor patient outcome, similar to observations with KLF4 ${ }^{100}$. KLF5 is also included in a 13 -gene signature comprised of genes important in embryonic stem characteristics that was associated with highly aggressive tumors, particularly enriched in basal-like breast carcinomas ${ }^{111}$.

In vitro studies have further characterized the association between KLF5 and proliferation. Reduction of KLF5 in breast cancer cells results in prolonged doubling times and reduced xenograft volumes ${ }^{59}$. Furthermore, enforced expression of KLF5 in MCF7 breast cancer cells enhanced tumor formation ${ }^{112}$. KLF5 partially exerts these mitogenic effects via upregulation of FGFBP1 $1^{59,112,113}$. FGFBP1 is a secreted protein which binds soluble and bound FGF ligands from the extracellular matrix and helps facilitate its binding to its respective receptor ${ }^{112}$. KLF5 depletion reduces FGFBP1 levels and FGFBP1 is sufficient to rescue the slow growth phenotype resulting from KLF5 suppression. Breast cancer cells disrupt the degradation of KLF5 through the expression of transcriptional co-activator TAZ. TAZ binds KLF5, 
protecting it from ubiquitination via E3-ligase WWP1 in ER-negative breast cancer cells ${ }^{59}$. This coexpression of KLF5 and TAZ was seen selectively in certain ER $\alpha$ negative cell lines, further contributing to the association of KLF5 and basal-like breast cancers. Expanding on this relationship, KLF5 facilitates progesterone-induced dedifferentiation of luminal, ER $\alpha$ positive T47D cells ${ }^{114}$. Progesterone treatment of T47D cells results in expression of basal-like breast cancer markers such as CK5 and suppression of KLF5 significantly inhibits this process. Furthermore, enforced expression of KLF5 in T47D cells induces CK5 mRNA expression. These results support KLF5 as a basal-like breast cancer marker. Interestingly, despite several protumorigenic functions in $\mathrm{ER} \alpha$ negative cell lines, some reports suggest that KLF5 can antagonize ER $\alpha$ signaling, possibility indicating unique functions for the transcription factor in the subtypes of breast cancer ${ }^{115}$.

\section{Classification and therapy of HER2+ breast cancers}

\section{Pathological and molecular classification of breast cancers}

In agreement with context-dependent roles for KLF4/5, the following dissertation describes novel functions for these factors in HER2-enriched breast cancer. Classically, breast cancers have been categorized based on the pathological markers ER $\alpha$, Progesterone receptor (PR), and HER2 (ErbB2). Typically, tumors which are positive for ER $\alpha$ by IHC are classified as ER+ and are subsequently treated with anti-hormonal therapy (IE, Tamoxifen) in conjunction with standard chemotherapies. Tumors that lack all three markers are considered triple-negative and are often highly aggressive tumors which to date lack suitable targeted therapies. In order to assess HER2 positivity, IHC is performed using scores $0-1+$ to indicate HER2-, a score of $2+$ is inconclusive, and a score of $3+$ is considered HER2+. The inconclusive $(2+)$ tumors are then 
tested for amplification of the ErbB2 gene via fluorescent in situ hybridization (FISH) to determine HER2 status. Expanding on the pathological definitions of breast cancer, additional studies have molecularly profiled this disease using microarrays generating the intrinsic subtypes of breast cancer: Luminal A, Luminal B, HER2-enriched, Basal-like, and Claudin-low ${ }^{116-118}$. These molecular portraits for the most part recapitulate the pathological definitions of breast cancer and may offer additionally insight to the genetic drivers of these subtypes.

Therapeutic strategies and limitations for HER2+ breast cancer

HER2 is a member of the membrane-spanning type I receptor tyrosine kinase (RTK) family $^{119}$. While experimental mutants of HER2 have demonstrated strong oncogenic abilities, the gene is most commonly expressed in human cancers as wild-type and such mutations are rarely found in human disease. Unlike other major RTKs, HER2 has no known natural ligand. Despite this fact, HER2 signaling is demonstrated in variety biological and pathological processes, ultimately impacting the Ras cascade and its principle effectors. HER2 has oncogenic functions in a diverse number of malignancies however, it has been most widely studied in breast cancer, where ErbB2 is amplified in $20-30 \%$ of the cases ${ }^{119}$. HER2+ breast cancers are highly proliferative and possess elevated metastatic capabilities. While historically HER2+ tumors were associated with poor prognoses, targeted therapies have greatly improved patient survival ${ }^{120-124}$.

The first of these targeted therapies was trastuzumab (Herceptin), a humanized monoclonal antibody to HER2. Trastuzumab acts by binding extracellular surface HER2, preventing homodimerization and subsequent activation of the intracellular tyrosine kinase (Figure. 6) ${ }^{125}$. In addition to this, antibody tagged cells can be cleared by the immune system, increasing the efficacy of this treatment. Pertruzumab, a newer monoclonal humanized antibody, 
acts similarly but also prevents heterodimerization with other RTKs namely HER3 ${ }^{125}$. These antibodies are often used alone or in combination with traditional cytotoxic chemotherapies in the neoadjuvant or adjuvant setting to treat HER2+ disease. A limitation of monoclonal antibodies is that they do not cross the blood-brain barrier and are unable to impact distal metastasis of the brain. Small molecule HER2/EGFR inhibitors, such as lapatinib (or the newer agent neratinib) have greater utility in this metastatic setting and can be used as third or fourth line therapy ${ }^{126}$. While small molecule inhibitors are effective in the adjuvant setting, often they fail to reach the same efficacy of the monoclonal antibodies when used alone. ${ }^{127}$ Ideally, patients should be treated with two anti-HER2 therapies with non-overlapping mechanisms and due to the high efficacy and more favorable side-effect profile, trastuzumab in combination with pertruzumab is preferred.

\section{Resistance to HER2-targeted therapies}

Despite the substantial therapeutic advance of HER2-targeted drugs, resistance to these therapies (intrinsic or developed) is commonly observed ${ }^{128-136}$. There are numerous mechanisms of resistance to trastuzumab. These include cleavage of the extracellular domain of HER2 or heterodimerization with other RTKs such as HER3 or IGFR1, ultimately achieving sustained activation of downstream signaling cascades such as MAPK and PI3K ${ }^{128,129,137}$. Maintaining the activity of these signaling pathways is a common node of resistance for either the monoclonal antibodies or small molecule treatments. There are a myriad of molecular perturbations which can accomplish this, for example PTEN loss or activating mutations in either the MAPK or PI3K pathways $^{131-134}$. Either as downstream Ras targets or from alternative signaling pathways, alterations such as CCNE amplification, c-SRC or mTOR activation can also convey resistance 
to these treatments ${ }^{135,136,138}$. Although there has been numerous experimentally validated mechanisms of resistance, it is unclear how this is accomplished in patients due to the scarcity tumor specimens of recurrent or resistant disease ${ }^{139}$.

The intrinsic apoptotic pathway

Although analyzing recurrent HER2+ breast cancers is difficult, researchers found elevated expression of PRKACA in resistant disease ${ }^{139}$. In an open reading frame kinase screen, the activated subunit of protein kinase A, PRKACA conveyed resistance to anti-HER2 therapies by neutralizing apoptotic signaling. Previously, other studies have also demonstrated vital roles for the intrinsic pathway of apoptosis in response to HER2 inhibition ${ }^{140-143}$.

The intrinsic pathway of apoptosis involves signaling networks that monitor cellular stresses such DNA damage, irradiation or reactive oxygen species (Figure. 7$)^{144,145}$. In response to these stresses, this pathway signals for the permeabilization of organelle membranes including the mitochondria, endoplasmic reticulum and lysosomes, releasing the noxious components of these organelles into the cytoplasm. These components (IE, reactive oxygen species, cytochrome c, $\mathrm{Ca}^{+2}$ ) can directly or indirectly mediate cell death via activation of caspases or caspaseindependent necrosis.

Many of the major regulators of the intrinsic pathways of apoptosis reside within the BCL2-family. The BCL2 family has at least 20 relatives, all containing at least one BCL2 homology $(\mathrm{BH})$ domain ${ }^{146}$. The anti-apoptotic proteins of this family, BCL2, BCL-XL, MCL1 and A1 contain all known BH domains, BH1-4. The pro-apoptotic, pore-forming proteins (Bak and Bax) lack the BH4 domain, however contain BH1-3 domains which are similar in sequence 
to those found in the anti-apoptotic molecules. The pro-apoptotic BH3-only members of the BCL2-family, for example Bik, Bim, Bad, Bid, and Noxa, contain only the BH3 domain as their name suggests.

As the anti-apoptotic and pore-forming, pro-apoptotic (also termed multi-domain proapoptotic) members of the family share domains BH1-3, they exhibit the same basic threedimensional protein structure ${ }^{146}$. This basic structure is comprised of a bundle of amphipathic $\alpha-$ helices surrounding an internal pair of hydrophobic $\alpha$-helices. Many of the members of the family interact functionally with each other via dimerization between BH3-domains. To regulate organelle membrane integrity the anti-apoptotic members bind the multi-domain pro-apoptotic members, essentially inactivating or sequestering these pore-forming proteins (Figure 8) ${ }^{146}$. When bound to an anti-apoptotic protein, Bak/Bax cannot undergo the homo/heterodimerization necessary for pore formation. Either through increased abundance or activation, BH3-only proteins can disrupt the protein-protein interaction between the anti-apopotic and multi-domain, pro-apoptotic molecules. Additionally, there is evidence that some BH3-only members can directly activate Bak/Bax. This ultimately allows for pore construction, compromising membrane integrity and expelling the internal organelle contents into the cytoplasm.

Anti-HER2 therapy and the intrinsic apoptotic pathway

While it is unclear whether through regulation via Ras or other signaling entities, the intrinsic apoptotic pathway plays a crucial role in anti-HER2 therapeutic efficacy. Overexpressing a constitutively active Bik (BikDD) significantly enhances the therapeutic response of HER2-amplified breast cancer cells to lapatinib treatment ${ }^{140}$. Furthermore, depletion of BCL2, BCL-XL, and/or MCL1 similarly enhances lapatinib-mediated cell death. In addition 
to BCL2 family members, depletion of alternative inhibitors of apoptosis such as survivin also increase the effect of lapatinib ${ }^{147}$. Moreover, cultured cells that have acquired resistance to either trastuzumab or lapatinib via long-term drug exposure increase levels of BCL-XL and $\operatorname{MCL1} 1^{148,149}$.

The principle concept from these studies indicates that the balance between the antiapoptotic and pro-apoptotic molecules can ultimately determine degree of therapeutic efficacy of HER2 inhibition. This concept has powerful clinical applications in both patient prognosis and therapy. Understanding the signaling networks that facilitate the modulation of apoptotic signaling would guide physicians towards more tailored treatment regimens, opting for more aggressive modalities if a patient was at high risk for therapeutic failure. Furthermore, combination therapy aimed at the anti-apoptotic molecules themselves may overcome resistance to anti-HER2 treatments and extend patient survival.

\section{Questions addressed in this dissertation}

KLF4 and KLF5 have been extensively studied in various physiological and pathophysiological processes. However, there are few reports investigating potential interactions between the two transcription factors in malignancy. This dissertation describes the relationship between KLF4 and KLF5 in breast tumorigenesis. Chapter 2 identifies and characterizes the positive correlation of the two KLFs in breast cancer. These two genes were identified as critical participants in resistance to HER2-targeted therapies through their promotion of anti-apoptotic signaling. Chapter 3 discusses how KLF4 and KLF5 interact in their respective effector 
pathways. Despite exerting redundant functions in mouse ES cells, KLF4 and KLF5 can exhibit overlapping, opposing, and distinct properties in breast cancer. Finally, Chapter 4 interprets these findings and the implications of this research on the respective fields, as well as addresses future work in this area. 


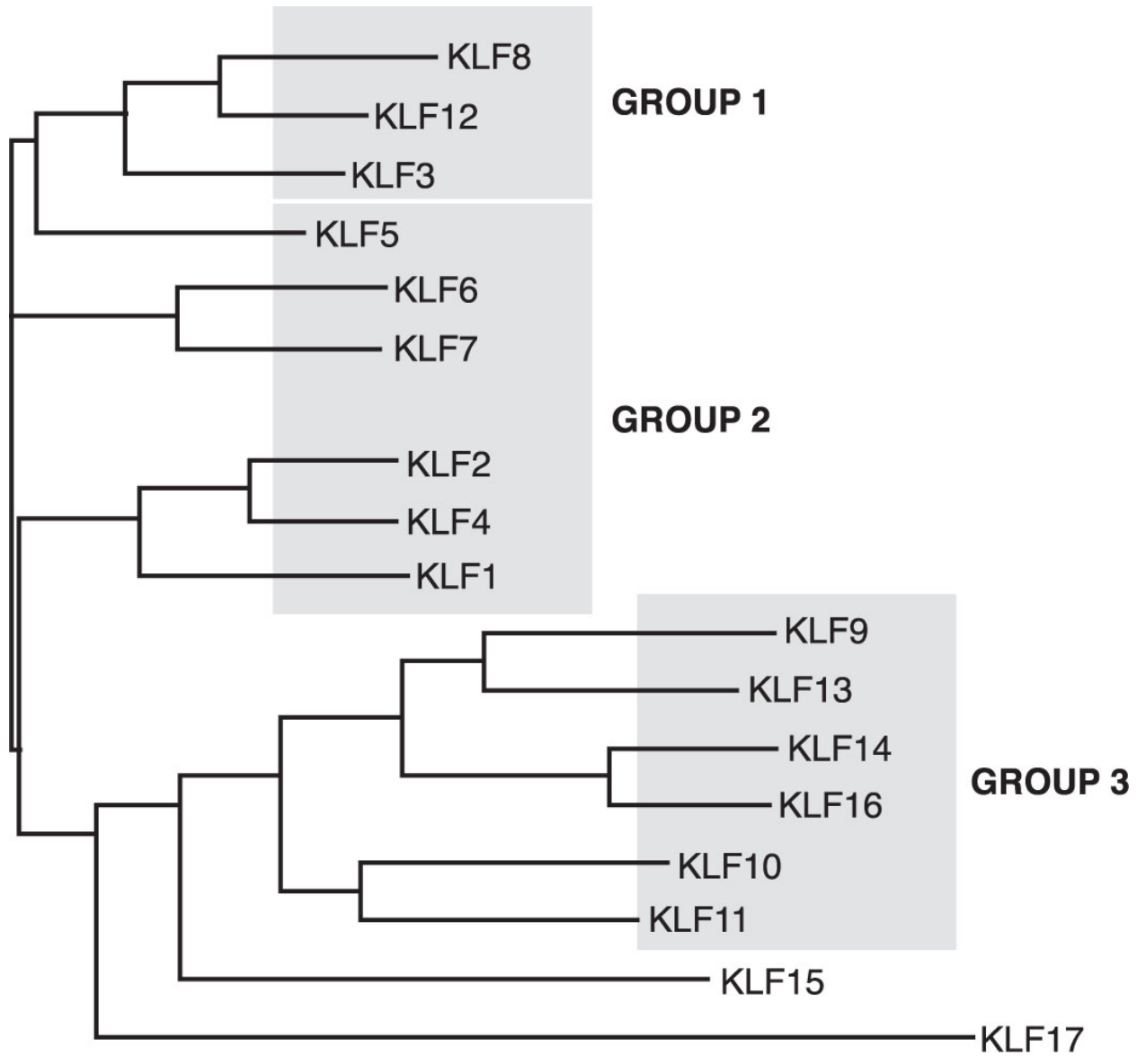

Figure 1. Phylogenetic tree of Kruppel-like factors. Adapted from McConnell et al. Physiol Rev. 90, 1337-1381 


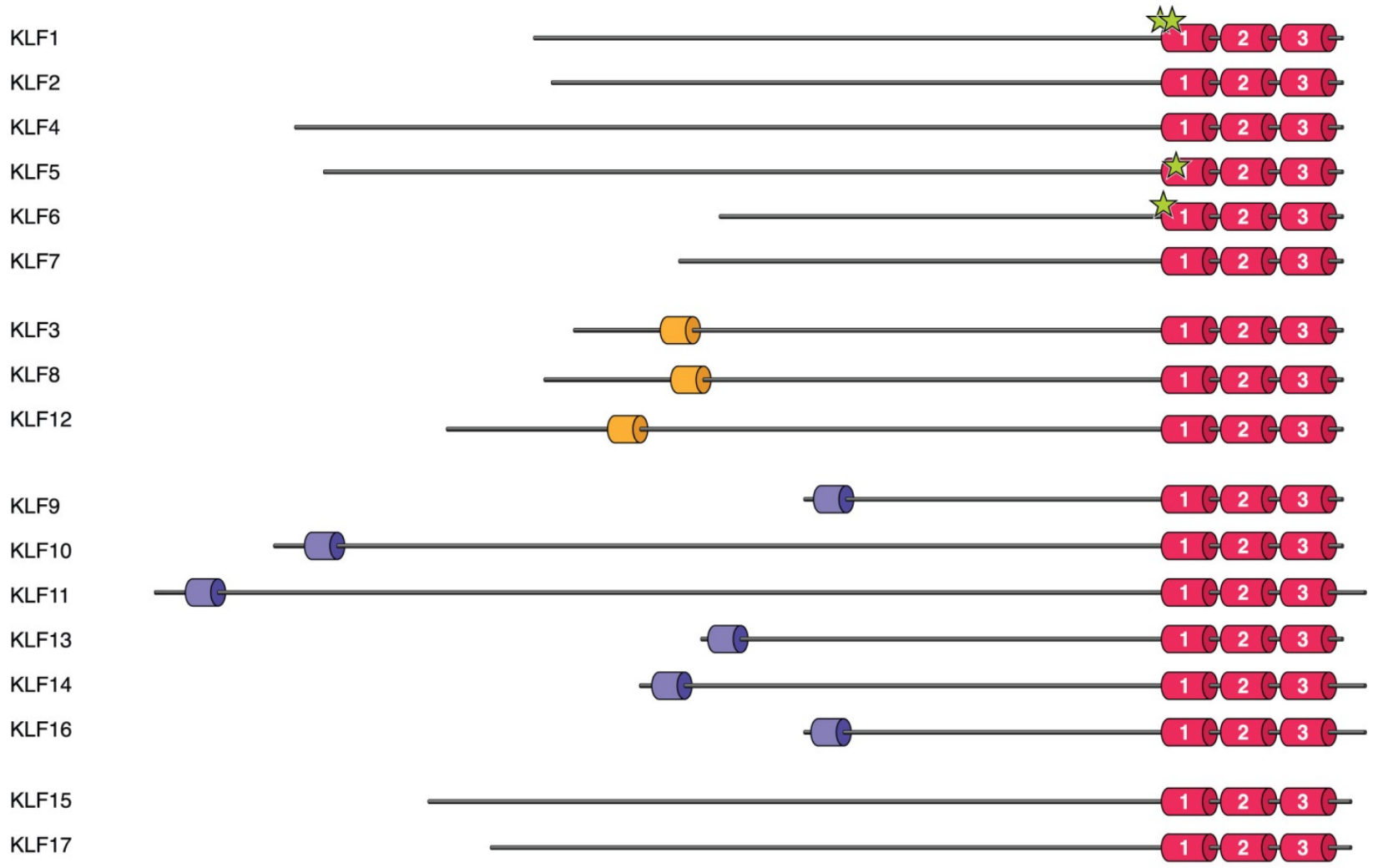

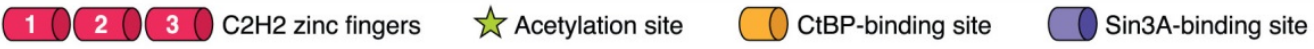

Figure 2. Protein structure of Kruppel-like factors. Adapted from McConnell et al. Physiol Rev. 90, 1337-1381 

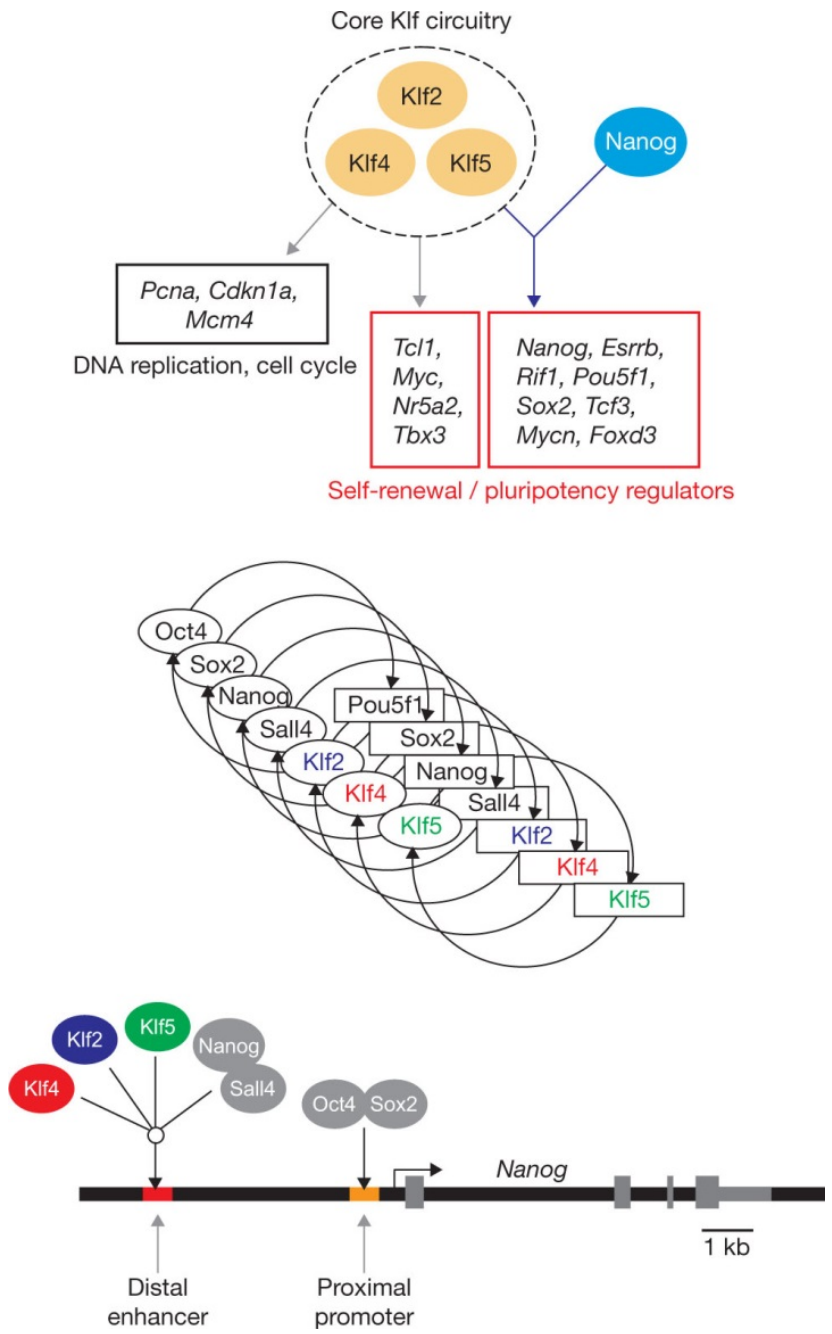

Figure 3. Core-KLF signaling in embryonic stem cells. Adapted from Jiang et al. Nat. Cell Biol. 10, 353-360 


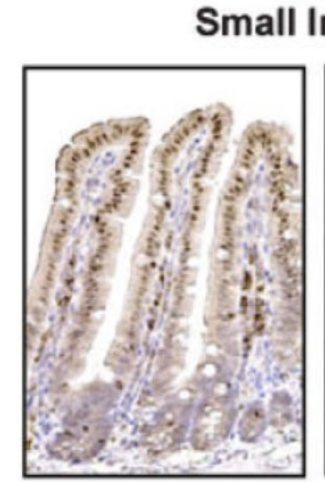

KIf4
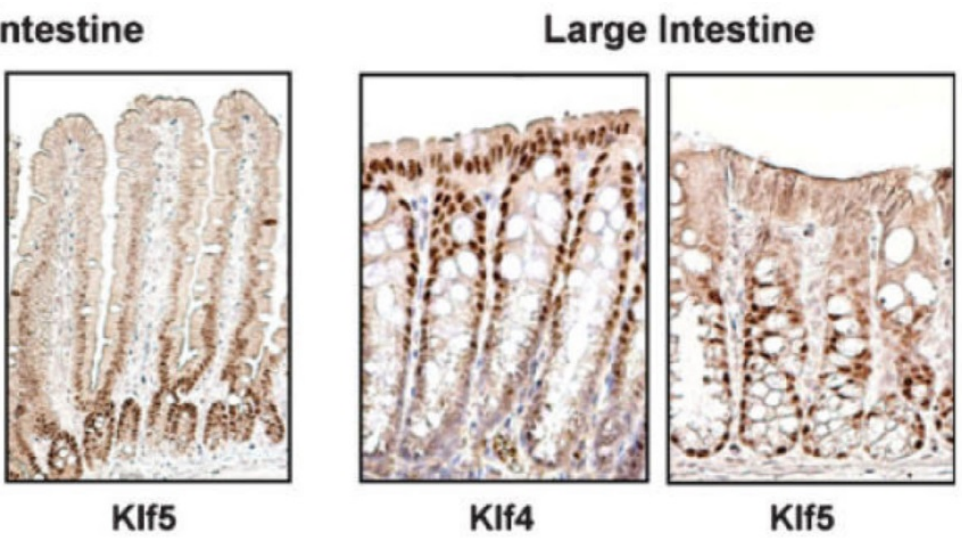

Figure 4. Immunostaining of KLF4 and KLF5 in gut epithelium. Adapted from McConnell et al. Bioessays 29, 549-557 


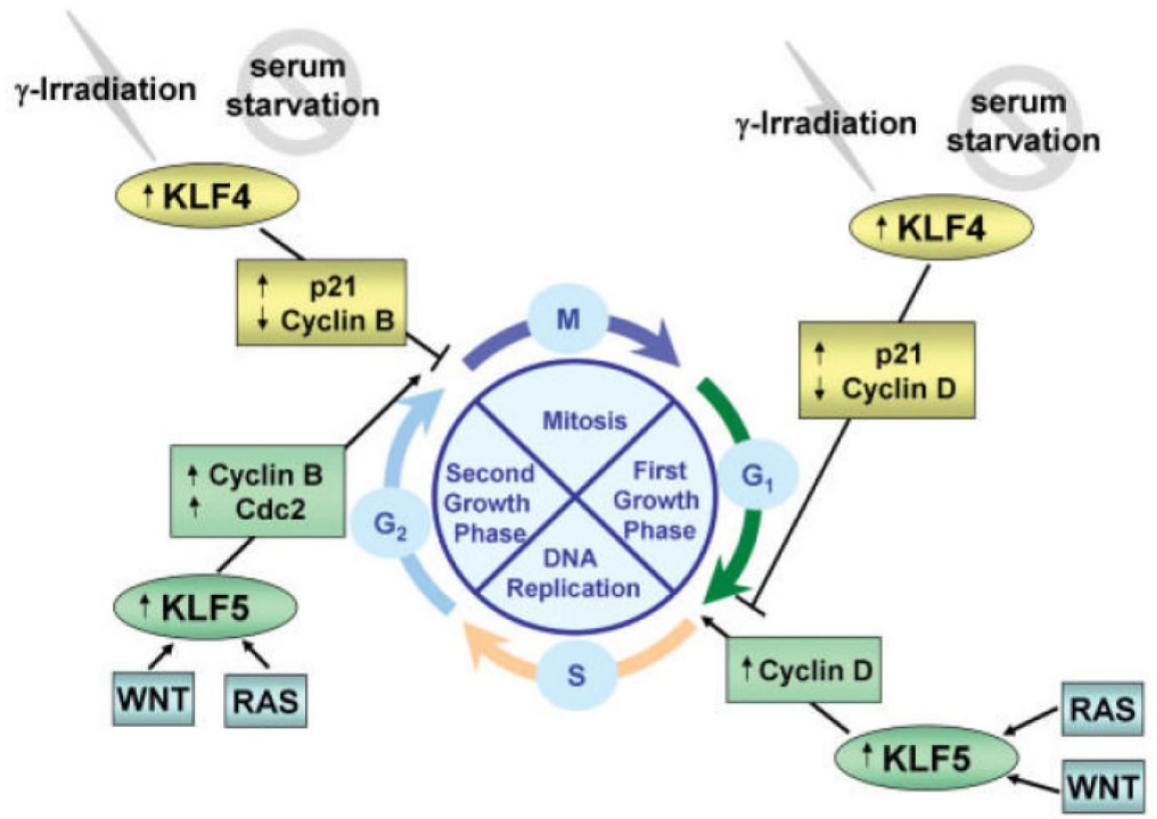

Figure 5. Cell cycle regulation of KLF4 and KLF5. Adapted from Ghaleb et al. Cell Res 15, 92-96 


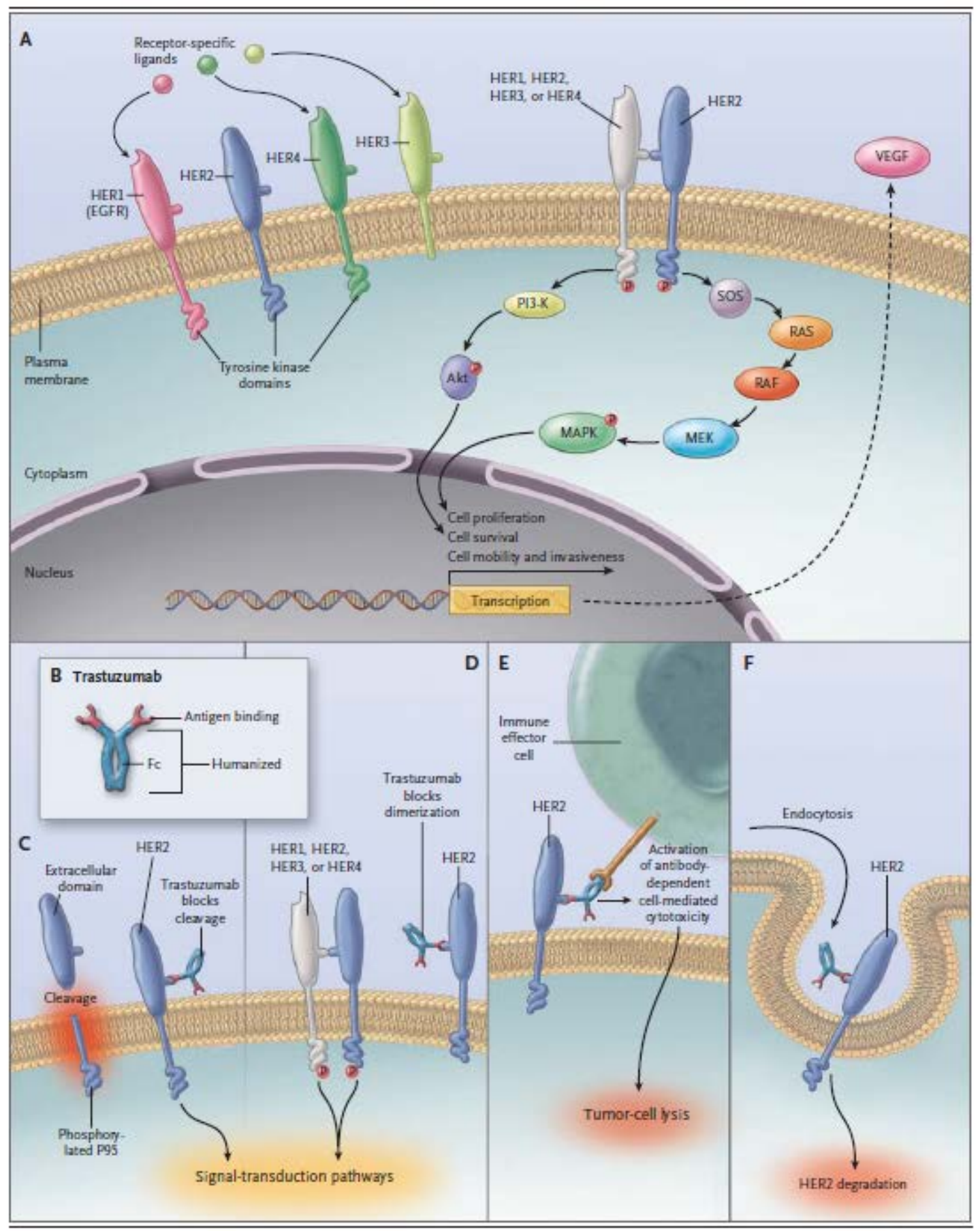

Figure 6. Mechanism of action by trastuzumab. A) Receptor tyrosine kinase signaling cascade B) Structure of the humanized monoclonal antibody to HER2, trastuzumab C,D) Mechanisms of action of trastuzumab E) Immune recruitment by trastuzumab F) Trastuzumab-mediated degradation of HER2. Adapted from Clifford A. Hudis $N$ Engl $J$ Med 2007 357, 39-51 


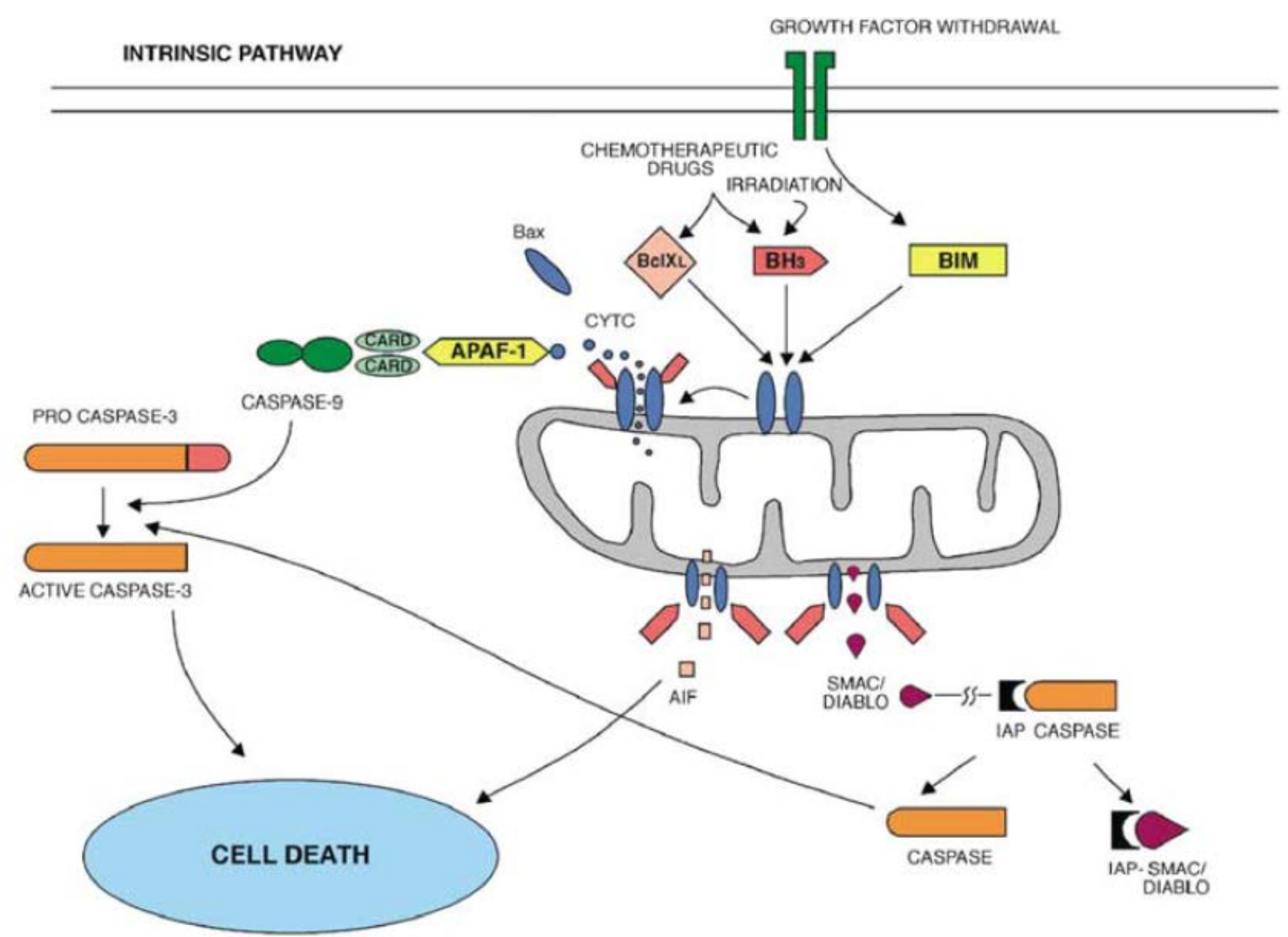

Figure 7. Intrinsic pathway of apoptosis. Adapted from U Tesla Leukemia 2004 18, 1176-1199 


\section{a MITOCHONDRIA}

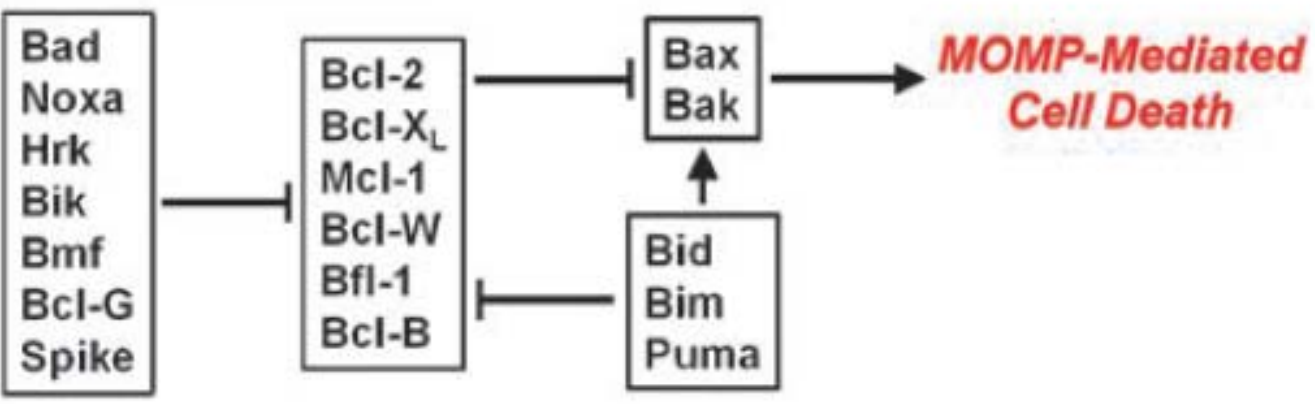

b ENDOPLASMIC RETICULUM (ER)

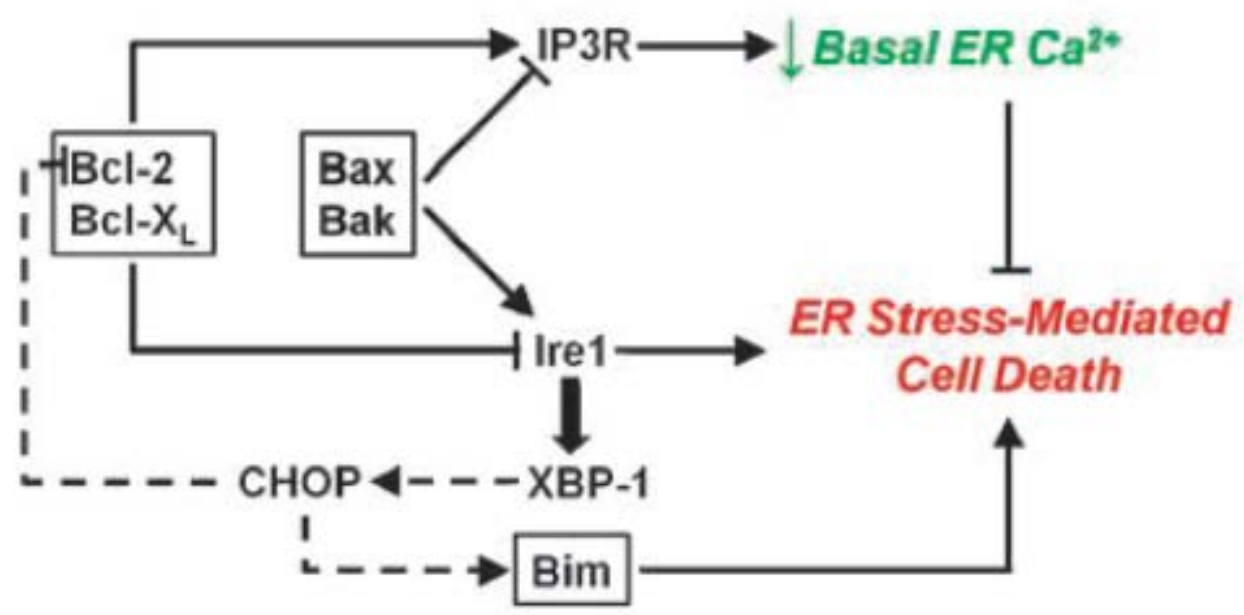

\section{c LYSOSOMES}

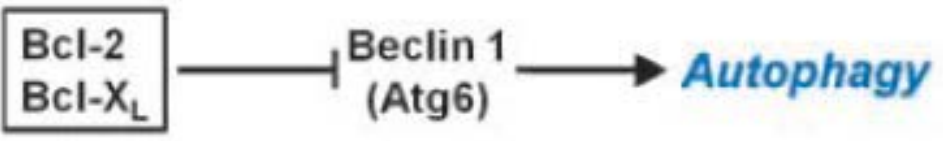

Figure 8. BCL2-family members and their roles in membrane integrity of the Mitochondria, ER and lysosome. Adapted from KW Yip and JC Reed Oncogene 2008 27, 6398-6406 


\section{Reference List}

1 McConnell BB, Yang,VW. Mammalian Kruppel-like factors in health and diseases. Physiol Rev 2010; 90: 1337-1381.

2 Schuh R, Aicher,W, Gaul,U, Cote,S, Preiss,A, Maier,D et al. A conserved family of nuclear proteins containing structural elements of the finger protein encoded by Kruppel, a Drosophila segmentation gene. Cell 1986; 47: 1025-1032.

3 Kadonaga JT, Carner,KR, Masiarz,FR, Tjian,R. Isolation of cDNA encoding transcription factor Sp1 and functional analys is of the DNA binding domain. Cell 1987; 51: 1079-1090.

4 Shields JM, Christy,RJ, Yang,VW. Identification and characterization of a gene encoding a gut-enriched Krüppel-like factor expressed during growth arrest. J Biol Chem 1996; 271: 20009-20017.

5 Sogawa K, Imataka,H, Yamasaki,Y, Kusume,H, Abe,H, Fujii-Kuriyama,Y. cDNA cloning and transcriptional properties of a novel GC box-binding protein, BTEB2. Nucleic Acids Res 1993; 21: 1527-1532.

6 Conkright MD, Wani,MA, Anderson,KP, Lingrel,JB. A gene encoding an intestinalenriched member of the Kruppel-like factor family expressed in intestinal epithelial cells. Nucleic Acids Res 1999; 27: 1263-1270.

7 Miller IJ, Bieker,JJ. A novel, erythroid cell-specific murine transcription factor that binds to the CACCC element and is related to the Krueppel family of nuclear proteins. Mol Cell Biol 1993; 13: 2776-2786.

8 Garrett-Sinha LA, Eberspaecher,H, Seldin,MF, de Crombrugghe,B. A gene for a novel zinc-finger protein expressed in differentiated epithelial cells and transiently in certain mesenchymal cells. J Biol Chem 1996; 271: 31384-31390. 
9 Ton-That H, Kaestner,KH, Shields,JM, Mahatanankoon,CS, Yang,VW. Expression of the gut-enriched Kruppel-like factor gene during development and intestinal tumorigenesis. FEBS Lett 1997; 419: 239-243.

10 Jenkins TD, Opitz,OG, Okano,J, Rustgi,AK. Transactivation of the human keratin 4 and Epstein-Barr virus ED-L2 promoters by gut-enriched Kruppel-like factor. $J$ Biol Chem 1998; 273: 10747-10754.

11 Panigada M, Porcellini,S, Sutti,F, Doneda,L, Pozzoli,O, Consalez,GG et al. GKLF in thymus epithelium as a developmentally regulated element of thymocyte-stroma cross-talk. Mech Dev 1999; 81: 103-113.

12 Fruman DA, Ferl,GZ, An,SS, Donahue,AC, Satterthwaite,AB, Witte,ON. Phosphoinositide 3-kinase and Bruton's tyrosine kinase regulate overlapping sets of genes in B lymphocytes. Proc Natl Acad Sci U S A 2002; 99: 359-364.

13 Hinnebusch BF, Siddique,A, Henderson,JW, Malo,MS, Zhang,W, Athaide,CP et al. Enterocyte differentiation marker intestinal alkaline phosphatase is a target gene of the gut-enriched Kruppel-like factor. Am J Physiol Gastrointest Liver Physiol 2004; 286: G23-G30.

14 Cullingford TE, Butler,MJ, Marshall,AK, Tham,eL, Sugden,PH, Clerk,A. Differential regulation of Kruppel-like factor family transcription factor expression in neonatal rat cardiac myocytes: effects of endothelin-1, oxidative stress and cytokines. Biochim Biophys Acta 2008; 1783: 1229-1236.

15 Sun R, Chen,X, Yang,VW. Intestinal-enriched Kruppel-like factor (Kruppel-like factor 5) is a positive regulator of cellular proliferation. J Biol Chem 2001; 276: 68976900.

16 Yet SF, McA'Nulty,MM, Folta,SC, Yen,HW, Yoshizumi,M, Hsieh,CM et al. Human EZF, a Kruppel-like zinc finger protein, is expressed in vascular endothelial cells and contains transcriptional activation and repression domains. J Biol Chem 1998; 273: $1026-1031$.

17 Zhang Z, Teng,CT. Phosphorylation of Kruppel-like factor 5 (KLF5/IKLF) at the CBP interaction region enhances its transactivation function. Nucleic Acids Res 2003; 31: 2196-2208. 
18 Miyamoto S, Suzuki,T, Muto,S, Aizawa,K, Kimura,A, Mizuno, Y et al. Positive and negative regulation of the cardiovascular transcription factor KLF5 by p300 and the oncogenic regulator SET through interaction and acetylation on the DNAbinding domain. Mol Cell Biol 2003; 23: 8528-8541.

19 Takahashi K, Yamanaka,S. Induction of pluripotent stem cells from mouse embryonic and adult fibroblast cultures by defined factors. Cell 2006; 126: 663-676.

20 Jiang J, Chan,YS, Loh,YH, Cai,J, Tong,GQ, Lim,CA et al. A core Klf circuitry regulates self-renewal of embryonic stem cells. Nat Cell Biol 2008; 10: 353-360.

21 Bourillot PY, Savatier,P. Kruppel-like transcription factors and control of pluripotency. BMC Biol 2010; 8: 125-.

22 Parisi S, Cozzuto,L, Tarantino,C, Passaro,F, Ciriello,S, Aloia,L et al. Direct targets of Klf5 transcription factor contribute to the maintenance of mouse embryonic stem cell undifferentiated state. BMC Biol 2010; 8: 128-.

23 Segre JA, Bauer,C, Fuchs,E. Klf4 is a transcription factor required for establishing the barrier function of the skin. Nat Genet 1999; 22: 356-360.

24 McConnell BB, Ghaleb,AM, Nandan,MO, Yang,VW. The diverse functions of Kruppellike factors 4 and 5 in epithelial biology and pathobiology. Bioessays 2007; 29: 549-557.

25 Jaubert J, Cheng,J, Segre,JA. Ectopic expression of kruppel like factor 4 (Klf4) accelerates formation of the epidermal permeability barrier. Development 2003; 130: 2767-2777.

26 Shindo T, Manabe,I, Fukushima,Y, Tobe,K, Aizawa,K, Miyamoto,S et al. Kruppel-like zinc-finger transcription factor KLF5/BTEB2 is a target for angiotensin II signaling and an essential regulator of cardiovascular remodeling. Nat Med 2002; 8: 856-863.

27 Ema M, Mori,D, Niwa,H, Hasegawa,Y, Yamanaka,Y, Hitoshi,S et al. Kruppel-like factor 5 is essential for blastocyst development and the normal self-renewal of mouse ESCs. Cell Stem Cell 2008; 3: 555-567. 
28 Parisi S, Russo,T. Regulatory role of Klf5 in early mouse development and in embryonic stem cells. Vitam Horm 2011; 87: 381-397.

29 Shinoda Y, Ogata,N, Higashikawa, A, Manabe,I, Shindo,T, Yamada,T et al. Kruppel-like factor 5 causes cartilage degradation through transactivation of matrix metalloproteinase 9. J Biol Chem 2008; 283: 24682-24689.

30 Oishi Y, Manabe,I, Tobe,K, Tsushima,K, Shindo,T, Fujiu,K et al. Kruppel-like transcription factor KLF5 is a key regulator of adipocyte differentiation. Cell Metab 2005; 1: 27-39.

31 Sur I, Rozell,B, Jaks,V, Bergstrom,A, Toftgard,R. Epidermal and craniofacial defects in mice overexpressing Klf5 in the basal layer of the epidermis. J Cell Sci 2006; 119: 3593-3601.

32 Swamynathan SK, Katz,JP, Kaestner,KH, Ashery-Padan,R, Crawford,MA, Piatigorsky,J. Conditional deletion of the mouse Klf4 gene results in corneal epithelial fragility, stromal edema, and loss of conjunctival goblet cells. Mol Cell Biol 2007; 27: 182194.

33 Kenchegowda D, Swamynathan,S, Gupta,D, Wan,H, Whitsett,J, Swamynathan,SK. Conditional disruption of mouse Klf5 results in defective eyelids with malformed meibomian glands, abnormal cornea and loss of conjunctival goblet cells. Dev Biol 2011; 356: 5-18.

34 Katz JP, Perreault,N, Goldstein,BG, Lee,CS, Labosky,PA, Yang,VW et al. The zincfinger transcription factor Klf4 is required for terminal differentiation of goblet cells in the colon. Development 2002; 129: 2619-2628.

35 Bell SM, Zhang,L, Xu,Y, Besnard,V, Wert,SE, Shroyer,N et al. Kruppel-like factor 5 controls villus formation and initiation of cytodifferentiation in the embryonic intestinal epithelium. Dev Biol 2013; 375: 128-139.

36 Watanabe N, Kurabayashi,M, Shimomura,Y, Kawai-Kowase,K, Hoshino,Y, Manabe,I et al. BTEB2, a Kruppel-like transcription factor, regulates expression of the SMemb/Nonmuscle myosin heavy chain B (SMemb/NMHC-B) gene. Circ Res 1999; 85: 182-191.

37 Hoshino Y, Kurabayashi,M, Kanda,T, Hasegawa,A, Sakamoto,H, Okamoto,E et al. Regulated expression of the BTEB2 transcription factor in vascular smooth 
muscle cells: analysis of developmental and pathological expression profiles shows implications as a predictive factor for restenosis. Circulation 2000; 102: 2528-2534.

38 Bafford R, Sui,XX, Wang,G, Conte,M. Angiotensin II and tumor necrosis factor-alpha upregulate survivin and Kruppel-like factor 5 in smooth muscle cells: Potential relevance to vein graft hyperplasia. Surgery 2006; 140: 289-296.

39 Ogata T, Kurabayashi,M, Hoshino,YI, Sekiguchi,KI, Kawai-Kowase,K, Ishikawa,S et al. Inducible expression of basic transcription factor-binding protein 2 (BTEB2), a member of zinc finger family of transcription factors, in cardiac allograft vascular disease. Transplantation 2000; 70: 1653-1656.

40 Ogata T, Kurabayashi,M, Hoshino,Y, Ishikawa,S, Takeyoshi,I, Morishita,Y et al. Inducible expression of BTEB2, a member of the zinc-finger family of transcription factors, in cardiac allograft arteriosclerosis. Transplant Proc 2000; 32: 2032-2033.

41 Sakamoto H, Sakamaki,T, Kanda,T, Hoshino,Y, Sawada,Y, Sato,M et al. Smooth muscle cell outgrowth from coronary atherectomy specimens in vitro is associated with less time to restenosis and expression of a key Transcription factor KLF5/BTEB2. Cardiology 2003; 100: 80-85.

42 Kawai-Kowase K, Kurabayashi,M, Hoshino,Y, Ohyama,Y, Nagai,R. Transcriptional activation of the zinc finger transcription factor BTEB2 gene by Egr-1 through mitogen-activated protein kinase pathways in vascular smooth muscle cells. Circ Res 1999; 85: 787-795.

43 Nagai R, Shindo,T, Manabe,I, Suzuki,T, Kurabayashi,M. KLF5/BTEB2, a Kruppel-like zinc-finger type transcription factor, mediates both smooth muscle cell activation and cardiac hypertrophy. Adv Exp Med Biol 2003; 538: 57-65.

44 Gao D, Niu,X, Ning,N, Hao,G. Regulation of angiotensin II-Induced Kruppel-like factor 5 expression in vascular smooth muscle cells. Biol Pharm Bull 2006; 29: 20042008.

45 He M, Han,M, Zheng,B, Shu,YN, Wen,JK. Angiotensin II stimulates KLF5 phosphorylation and its interaction with c-Jun leading to suppression of p21 expression in vascular smooth muscle cells. J Biochem 2009; 146: 683-691. 
46 Adam PJ, Regan,CP, Hautmann, MB, Owens,GK. Positive- and negative-acting Kruppellike transcription factors bind a transforming growth factor beta control element required for expression of the smooth muscle cell differentiation marker SM22alpha in vivo. J Biol Chem 2000; 275: 37798-37806.

47 Yoshida T, Gan,Q, Owens,GK. Kruppel-like factor 4, Elk-1, and histone deacetylases cooperatively suppress smooth muscle cell differentiation markers in response to oxidized phospholipids. Am J Physiol Cell Physiol 2008; 295: C1175-C1182.

48 Yoshida T, Kaestner,KH, Owens,GK. Conditional deletion of Kruppel-like factor 4 delays downregulation of smooth muscle cell differentiation markers but accelerates neointimal formation following vascular injury. Circ Res 2008; 102: 1548-1557.

49 Pidkovka NA, Cherepanova,OA, Yoshida,T, Alexander,MR, Deaton,RA, Thomas,JA et al. Oxidized phospholipids induce phenotypic switching of vascular smooth muscle cells in vivo and in vitro. Circ Res 2007; 101: 792-801.

50 Cherepanova OA, Pidkovka,NA, Sarmento,OF, Yoshida,T, Gan,Q, Adiguzel,E et al. Oxidized phospholipids induce type VIII collagen expression and vascular smooth muscle cell migration. Circ Res 2009; 104: 609-618.

51 Wassmann S, Wassmann,K, Jung,A, Velten,M, Knuefermann,P, Petoumenos,V et al. Induction of p53 by GKLF is essential for inhibition of proliferation of vascular smooth muscle cells. J Mol Cell Cardiol 2007; 43: 301-307.

52 Wang C, Han,M, Zhao,XM, Wen,JK. Kruppel-like factor 4 is required for the expression of vascular smooth muscle cell differentiation marker genes induced by all-trans retinoic acid. J Biochem 2008; 144: 313-321.

53 Zheng B, Han,M, Bernier,M, Zhang,XH, Meng,F, Miao,SB et al. Kruppel-like factor 4 inhibits proliferation by platelet-derived growth factor receptor beta-mediated, not by retinoic acid receptor alpha-mediated, phosphatidylinositol 3-kinase and ERK signaling in vascular smooth muscle cells. J Biol Chem 2009; 284: 22773-22785.

54 Liu Y, Sinha,S, McDonald,OG, Shang,Y, Hoofnagle,MH, Owens,GK. Kruppel-like factor 4 abrogates myocardin-induced activation of smooth muscle gene expression. J Biol Chem 2005; 280: 9719-9727. 
55 Cordes KR, Sheehy,NT, White,MP, Berry,EC, Morton,SU, Muth,AN et al. miR-145 and miR-143 regulate smooth muscle cell fate and plasticity. Nature 2009; 460: 705710.

56 Cheng Y, Liu,X, Yang,J, Lin,Y, Xu,DZ, Lu,Q et al. MicroRNA-145, a novel smooth muscle cell phenotypic marker and modulator, controls vascular neointimal lesion formation. Circ Res 2009; 105: 158-166.

57 Ghaleb AM, Nandan,MO, Chanchevalap,S, Dalton,WB, Hisamuddin,IM, Yang,VW. Kruppel-like factors 4 and 5: the yin and yang regulators of cellular proliferation. Cell Res 2005; 15: 92-96.

58 Yori JL, Johnson,E, Zhou,G, Jain,MK, Keri,RA. Kruppel-like factor 4 inhibits epithelialto-mesenchymal transition through regulation of E-cadherin gene expression. $J$ Biol Chem 2010; 285: 16854-16863.

59 Zhao D, Zhi,X, Zhou,Z, Chen,C. TAZ antagonizes the WWP1-mediated KLF5 degradation and promotes breast cell proliferation and tumorigenesis. Carcinogenesis 2012; 33: 59-67.

60 Yoon HS, Chen,X, Yang,VW. Kruppel-like factor 4 mediates p53-dependent G1/S cell cycle arrest in response to DNA damage. J Biol Chem 2003; 278: 2101-2105.

61 Yoon HS, Ghaleb,AM, Nandan,MO, Hisamuddin,IM, Dalton,WB, Yang,VW. Kruppellike factor 4 prevents centrosome amplification following gamma-irradiationinduced DNA damage. Oncogene 2005; 24: 4017-4025.

62 Yoon HS, Yang,VW. Requirement of Kruppel-like factor 4 in preventing entry into mitosis following DNA damage. J Biol Chem 2004; 279: 5035-5041.

63 Nandan MO, Yoon,HS, Zhao,W, Ouko,LA, Chanchevalap,S, Yang,VW. Kruppel-like factor 5 mediates the transforming activity of oncogenic H-Ras. Oncogene 2004; 23: $3404-3413$.

64 Nandan MO, Ghaleb,AM, McConnell,BB, Patel,NV, Robine,S, Yang,VW. Kruppel-like factor 5 is a crucial mediator of intestinal tumorigenesis in mice harboring combined ApcMin and KRASV12 mutations. Mol Cancer 2010; 9: 63-. 
65 Chen C, Benjamin,MS, Sun,X, Otto,KB, Guo,P, Dong,XY et al. KLF5 promotes cell proliferation and tumorigenesis through gene regulation and the TSU-Pr1 human bladder cancer cell line. Int J Cancer 2006; 118: 1346-1355.

66 Ghaleb AM, Katz,JP, Kaestner,KH, Du,JX, Yang,VW. Kruppel-like factor 4 exhibits antiapoptotic activity following gamma-radiation-induced DNA damage. Oncogene 2007; 26: 2365-2373.

67 Rowland BD, Peeper,DS. KLF4, p21 and context-dependent opposing forces in cancer. Nat Rev Cancer 2006; 6: 11-23.

68 Rowland BD, Bernards,R, Peeper,DS. The KLF4 tumour suppressor is a transcriptional repressor of p53 that acts as a context-dependent oncogene. Nat Cell Biol 2005; 7: 1074-1082.

69 Zhu N, Gu,L, Findley,HW, Chen,C, Dong,JT, Yang,L et al. KLF5 Interacts with p53 in regulating survivin expression in acute lymphoblastic leukemia. J Biol Chem 2006; 281: 14711-14718.

70 Zhao Y, Hamza,MS, Leong,HS, Lim,CB, Pan,YF, Cheung,E et al. Kruppel-like factor 5 modulates p53-independent apoptosis through Pim1 survival kinase in cancer cells. Oncogene 2008; 27: 1-8.

71 Suzuki T, Nishi,T, Nagino,T, Sasaki,K, Aizawa,K, Kada,N et al. Functional interaction between the transcription factor Kruppel-like factor 5 and poly(ADP-ribose) polymerase-1 in cardiovascular apoptosis. J Biol Chem 2007; 282: 9895-9901.

72 Tong D, Czerwenka,K, Heinze,G, Ryffel,M, Schuster,E, Witt,A et al. Expression of KLF5 is a prognostic factor for disease-free survival and overall survival in patients with breast cancer. Clin Cancer Res 2006; 12: 2442-2448.

73 Noto JM, Khizanishvili, T, Chaturvedi,R, Piazuelo,MB, Romero-Gallo,J, Delgado,AG et al. Helicobacter pylori promotes the expression of Kruppel-like factor 5, a mediator of carcinogenesis, in vitro and in vivo. PLoS One 2013; 8: e54344-.

74 Zhang W, Geiman,DE, Shields,JM, Dang,DT, Mahatan,CS, Kaestner,KH et al. The gutenriched Kruppel-like factor (Kruppel-like factor 4) mediates the transactivating effect of p53 on the p21WAF1/Cip1 promoter. J Biol Chem 2000; 275: 1839118398. 
75 Ghaleb AM, McConnell,BB, Nandan,MO, Katz,JP, Kaestner,KH, Yang,VW. Haploinsufficiency of Kruppel-like factor 4 promotes adenomatous polyposis coli dependent intestinal tumorigenesis. Cancer Res 2007; 67: 7147-7154.

76 Barker N, Ridgway, RA, van Es,JH, van de,WM, Begthel,H, van den,BM et al. Crypt stem cells as the cells-of-origin of intestinal cancer. Nature 2009; 457: 608-611.

77 Radtke F, Clevers,H. Self-renewal and cancer of the gut: two sides of a coin. Science 2005; 307: 1904-1909.

78 Scoville DH, Sato,T, He,XC, Li,L. Current view: intestinal stem cells and signaling. Gastroenterology 2008; 134: 849-864.

79 Stone CD, Chen,ZY, Tseng,CC. Gut-enriched Kruppel-like factor regulates colonic cell growth through APC/beta-catenin pathway. FEBS Lett 2002; 530: 147-152.

80 Zhang W, Chen,X, Kato,Y, Evans,PM, Yuan,S, Yang,J et al. Novel cross talk of Kruppel-like factor 4 and beta-catenin regulates normal intestinal homeostasis and tumor repression. Mol Cell Biol 2006; 26: 2055-2064.

81 Dang DT, Chen,X, Feng,J, Torbenson,M, Dang,LH, Yang,VW. Overexpression of Kruppel-like factor 4 in the human colon cancer cell line RKO leads to reduced tumorigenecity. Oncogene 2003; 22: 3424-3430.

82 Leng Z, Tao,K, Xia,Q, Tan,J, Yue,Z, Chen,J et al. Kruppel-like factor 4 acts as an oncogene in colon cancer stem cell-enriched spheroid cells. PLoS One 2013; 8: e56082-.

83 Du JX, Bialkowska,AB, McConnell,BB, Yang,VW. SUMOylation regulates nuclear localization of Kruppel-like factor 5. J Biol Chem 2008; 283: 31991-32002.

84 Nandan MO, Chanchevalap,S, Dalton,WB, Yang,VW. Kruppel-like factor 5 promotes mitosis by activating the cyclin B1/Cdc2 complex during oncogenic Ras-mediated transformation. FEBS Lett 2005; 579: 4757-4762.

85 Nandan MO, McConnell,BB, Ghaleb,AM, Bialkowska,AB, Sheng,H, Shao,J et al. Kruppel-like factor 5 mediates cellular transformation during oncogenic KRASinduced intestinal tumorigenesis. Gastroenterology 2008; 134: 120-130. 
86 McConnell BB, Bialkowska,AB, Nandan,MO, Ghaleb,AM, Gordon,FJ, Yang,VW. Haploinsufficiency of Kruppel-like factor 5 rescues the tumor-initiating effect of the Apc(Min) mutation in the intestine. Cancer Res 2009; 69: 4125-4133.

87 Nakaya T, Ogawa,S, Manabe,I, Tanaka,M, Sanada,M, Sato,T et al. KLF5 regulates the integrity and oncogenicity of intestinal stem cells. Cancer Res 2014; 74: 28822891.

88 Kwak MK, Lee,HJ, Hur,K, Park,dJ, Lee,HS, Kim,WH et al. Expression of Kruppel-like factor 5 in human gastric carcinomas. J Cancer Res Clin Oncol 2008; 134: 163167.

89 Foster KW, Ren,S, Louro,ID, Lobo-Ruppert,SM, McKie-Bell,P, Grizzle,W et al. Oncogene expression cloning by retroviral transduction of adenovirus E1Aimmortalized rat kidney RK3E cells: transformation of a host with epithelial features by c-MYC and the zinc finger protein GKLF. Cell Growth Differ 1999; 10: $423-434$.

90 Foster KW, Liu,Z, Nail,CD, Li,X, Fitzgerald,TJ, Bailey,SK et al. Induction of KLF4 in basal keratinocytes blocks the proliferation-differentiation switch and initiates squamous epithelial dysplasia. Oncogene 2005; 24: 1491-1500.

91 Huang CC, Liu,Z, Li,X, Bailey,SK, Nail,CD, Foster,KW et al. KLF4 and PCNA identify stages of tumor initiation in a conditional model of cutaneous squamous epithelial neoplasia. Cancer Biology and Therapy 2005; 4: 1401-1408.

92 Jiang W, Deng,W, Bailey,SK, Nail,CD, Frost,AR, Brouillette,WJ et al. Prevention of KLF4-mediated tumor initiation and malignant transformation by UAB30 rexinoid. Cancer Biol Ther 2009; 8: 287-296.

93 Abrigo M, Alvarez,R, Paparella,ML, Calb,DE, Bal de Kier,JE, Gutkind,JS et al. Impairing squamous differentiation by Klf4 deletion is sufficient to initiate tongue carcinoma development upon K-Ras activation in mice. Carcinogenesis 2014; 35: 662-669.

94 Demehri S, Turkoz,A, Kopan,R. Epidermal Notch1 loss promotes skin tumorigenesis by impacting the stromal microenvironment. Cancer Cell 2009; 16: 55-66. 
95 Mao X, Miao,S, He,H, Miao,S, Pei,R, Yang,X et al. Kruppel-like factor 5: a novel biomarker for lymph node metastasis and recurrence in supraglottic squamous cell laryngeal carcinoma. Tumour Biol 2014; 35: 623-629.

96 Foster KW, Frost,AR, McKie-Bell,P, Lin,CY, Engler,JA, Grizzle,WE et al. Increase of GKLF messenger RNA and protein expression during progression of breast cancer. Cancer Res 2000; 60: 6488-6495.

97 Pandya AY, Talley,LI, Frost,AR, Fitzgerald,TJ, Trivedi,V, Chakravarthy,M et al. Nuclear localization of KLF4 is associated with an aggressive phenotype in earlystage breast cancer. Clin Cancer Res 2004; 10: 2709-2719.

98 Chu PY, Hsu,NC, Liao,AT, Yeh,KT, Hou,MF, Liu,CH. Elevated Kruppel-like factor 4 transcription factor in canine mammary carcinoma. BMC Vet Res 2011; 7: 58-.

99 Chen CJ, Lin,SE, Lin, YM, Lin,SH, Chen,DR, Chen,CL. Association of expression of Kruppel-like Factor 4 and Kruppel-like Factor 5 with the clinical manifestations of breast cancer. Pathol Oncol Res 2012; 18: 161-168.

100 Kamalakaran S, Varadan,V, Giercksky Russnes,HE, Levy,D, Kendall,J, Janevski,A et al. DNA methylation patterns in luminal breast cancers differ from non-luminal subtypes and can identify relapse risk independent of other clinical variables. Mol Oncol 2011; 5: 77-92.

101 Akaogi K, Nakajima,Y, Ito,I, Kawasaki,S, Oie,SH, Murayama,A et al. KLF4 suppresses estrogen-dependent breast cancer growth by inhibiting the transcriptional activity of ERa. Oncogene 2009; 28: 2894-2902.

102 Tiwari N, Meyer-Schaller,N, Arnold,P, Antoniadis,H, Pachkov,M, van Nimwegen,E et al. Klf4 is a transcriptional regulator of genes critical for EMT, including Jnk1 (Mapk8). PLoS One 2013; 8: e57329-.

103 Yori JL, Seachrist,DD, Johnson,E, Lozada,KL, Abdul-Karim,FW, Chodosh,LA et al. Kruppel-like factor 4 inhibits tumorigenic progression and metastasis in a mouse model of breast cancer. Neoplasia 2011; 13: 601-610.

104 Liu Z, Teng,L, Bailey,SK, Frost,AR, Bland,KI, Lobuglio,AF et al. Epithelial transformation by KLF4 requires Notch1 but not canonical Notch1 signaling. Cancer Biol Ther 2009; 8: 1840-1851. 
$105 \mathrm{Yu}$ F, Li,J, Chen,H, Fu,J, Ray,S, Huang,S et al. Kruppel-like factor 4 (KLF4) is required for maintenance of breast cancer stem cells and for cell migration and invasion. Oncogene 2011; 30: 2161-2172.

106 Lin CC, Liu,LZ, Addison,JB, Ivanov,AV, Ruppert,JM. A KLF4-miRNA-206 autoregulatory feedback loop can promote or inhibit protein translation depending upon cell context. Mol Cell Biol 2011; 31: 2513-2527.

107 Yu F, Shi,Y, Wang,J, Li,J, Fan,D, Ai,W. Deficiency of Kruppel-like factor KLF4 in mammary tumor cells inhibits tumor growth and pulmonary metastasis and is accompanied by compromised recruitment of myeloid-derived suppressor cells. Int J Cancer 2013; 133: 2872-2883.

108 Cittelly DM, Finlay-Schultz,J, Howe,EN, Spoelstra,NS, Axlund,SD, Hendricks,P et al. Progestin suppression of miR-29 potentiates dedifferentiation of breast cancer cells via KLF4. Oncogene 2012; 32: 2555-2564.

109 Hu D, Zhou,Z, Davidson,NE, Huang,Y, Wan,Y. Novel insight into KLF4 proteolytic regulation in estrogen receptor signaling and breast carcinogenesis. J Biol Chem 2012; 287: 13584-13597.

110 Chen C, Bhalala,HV, Qiao,H, Dong,JT. A possible tumor suppressor role of the KLF5 transcription factor in human breast cancer. Oncogene 2002; 21: 6567-6572.

111 Ben-Porath I, Thomson,MW, Carey,VJ, Ge,R, Bell,GW, Regev,A et al. An embryonic stem cell-like gene expression signature in poorly differentiated aggressive human tumors. Nat Genet 2008; 40: 499-507.

112 Zheng HQ, Zhou,Z, Huang,J, Chaudhury,L, Dong,JT, Chen,C. Kruppel-like factor 5 promotes breast cell proliferation partially through upregulating the transcription of fibroblast growth factor binding protein 1. Oncogene 2009; 28: 3702-3713.

113 Liu R, Zheng,HQ, Zhou,Z, Dong,JT, Chen,C. KLF5 promotes breast cell survival partially through fibroblast growth factor-binding protein 1-pERK-mediated dual specificity MKP-1 protein phosphorylation and stabilization. J Biol Chem 2009; 284: 16791-16798.

114 Liu R, Zhou,Z, Zhao,D, Chen,C. The induction of KLF5 transcription factor by progesterone contributes to progesterone-induced breast cancer cell proliferation and dedifferentiation. Mol Endocrinol 2011; 25: 1137-1144. 
115 Zhao KW, Sikriwal,D, Dong,X, Guo,P, Sun,X, Dong,JT. Oestrogen causes degradation of KLF5 by inducing the E3 ubiquitin ligase EFP in ER-positive breast cancer cells. Biochem J 2011; 437: 323-333.

116 Parker JS, Mullins,M, Cheang,MC, Leung,S, Voduc,D, Vickery,T et al. Supervised risk predictor of breast cancer based on intrinsic subtypes. J Clin Oncol 2009; 27: 1160-1167.

117 Perou CM, Sorlie,T, Eisen,MB, van de,RM, Jeffrey,SS, Rees,CA et al. Molecular portraits of human breast tumours. Nature 2000; 406: 747-752.

118 Prat A, Parker,JS, Karginova,O, Fan,C, Livasy,C, Herschkowitz,JI et al. Phenotypic and molecular characterization of the claudin-low intrinsic subtype of breast cancer. Breast Cancer Res 2010; 12: R68-.

119 Harari D, Yarden,Y. Molecular mechanisms underlying ErbB2/HER2 action in breast cancer. Oncogene 2000; 19: 6102-6114.

120 Blackwell KL, Pegram,MD, Tan-Chiu,E, Schwartzberg,LS, Arbushites,MC, Maltzman,JD et al. Single-agent lapatinib for HER2-overexpressing advanced or metastatic breast cancer that progressed on first- or second-line trastuzumabcontaining regimens. Ann Oncol 2009; 20: 1026-1031.

121 Cobleigh MA, Vogel,CL, Tripathy,D, Robert,NJ, Scholl,S, Fehrenbacher,L et al. Multinational study of the efficacy and safety of humanized anti-HER2 monoclonal antibody in women who have HER2-overexpressing metastatic breast cancer that has progressed after chemotherapy for metastatic disease. J Clin Oncol 1999; 17: 2639-2648.

122 Vogel C, Cobleigh,MA, Tripathy,D, Gutheil,JC, Harris,LN, Fehrenbacher,L et al. Firstline, single-agent Herceptin(R) (trastuzumab) in metastatic breast cancer. a preliminary report. Eur J Cancer 2001; 37 Suppl 1: 25-29.

123 Vogel CL, Cobleigh,MA, Tripathy,D, Gutheil,JC, Harris,LN, Fehrenbacher,L et al. Efficacy and safety of trastuzumab as a single agent in first-line treatment of HER2-overexpressing metastatic breast cancer. J Clin Oncol 2002; 20: 719-726.

124 Piccart-Gebhart MJ, Procter,M, Leyland-Jones,B, Goldhirsch,A, Untch,M, Smith,I et al. Trastuzumab after adjuvant chemotherapy in HER2-positive breast cancer. $N$ Engl J Med 2005; 353: 1659-1672. 
125 Hudis CA. Trastuzumab--mechanism of action and use in clinical practice. $N$ Engl $J$ Med 2007; 357: 39-51.

126 Burstein HJ, Sun,Y, Dirix,LY, Jiang,Z, Paridaens,R, Tan,AR et al. Neratinib, an irreversible ErbB receptor tyrosine kinase inhibitor, in patients with advanced ErbB2-positive breast cancer. J Clin Oncol 2010; 28: 1301-1307.

127 Pernas SS. Neoadjuvant therapy of early stage human epidermal growth factor receptor 2 positive breast cancer: latest evidence and clinical implications. Ther Adv Med Oncol 2014; 6: 210-221.

128 Xia W, Liu,LH, Ho,P, Spector,NL. Truncated ErbB2 receptor (p95ErbB2) is regulated by heregulin through heterodimer formation with ErbB3 yet remains sensitive to the dual EGFR/ErbB2 kinase inhibitor GW572016. Oncogene 2004; 23: 646-653.

129 Nahta R, Yuan,LX, Du,Y, Esteva,FJ. Lapatinib induces apoptosis in trastuzumabresistant breast cancer cells: effects on insulin-like growth factor I signaling. Mol Cancer Ther 2007; 6: 667-674.

130 Nagata Y, Lan,KH, Zhou,X, Tan,M, Esteva,FJ, Sahin,AA et al. PTEN activation contributes to tumor inhibition by trastuzumab, and loss of PTEN predicts trastuzumab resistance in patients. Cancer Cell 2004; 6: 117-127.

131 Berns K, Horlings,HM, Hennessy,BT, Madiredjo,M, Hijmans,EM, Beelen,K et al. A functional genetic approach identifies the PI3K pathway as a major determinant of trastuzumab resistance in breast cancer. Cancer Cell 2007; 12: 395-402.

132 Kataoka Y, Mukohara,T, Shimada,H, Saijo,N, Hirai,M, Minami,H. Association between gain-of-function mutations in PIK3CA and resistance to HER2-targeted agents in HER2-amplified breast cancer cell lines. Ann Oncol 2010; 21: 255-262.

133 Esteva FJ, Guo,H, Zhang,S, Santa-Maria,C, Stone,S, Lanchbury,JS et al. PTEN, PIK3CA, p-AKT, and p-p70S6K status: association with trastuzumab response and survival in patients with HER2-positive metastatic breast cancer. Am J Pathol 2010; 177: 1647-1656.

134 Dave B, Migliaccio,I, Gutierrez,MC, Wu,MF, Chamness,GC, Wong, $\mathrm{H}$ et al. Loss of phosphatase and tensin homolog or phosphoinositol-3 kinase activation and response to trastuzumab or lapatinib in human epidermal growth factor receptor 
2-overexpressing locally advanced breast cancers. J Clin Oncol 2011; 29: 166173.

135 Scaltriti M, Eichhorn,PJ, Cortes,J, Prudkin,L, Aura,C, Jimenez,J et al. Cyclin E amplification/overexpression is a mechanism of trastuzumab resistance in HER2+ breast cancer patients. Proc Natl Acad Sci U S A 2011; 108: 3761-3766.

136 Zhang S, Huang,WC, Li,P, Guo,H, Poh,SB, Brady,SW et al. Combating trastuzumab resistance by targeting SRC, a common node downstream of multiple resistance pathways. Nat Med 2011; 17: 461-469.

137 Wehrman TS, Raab,WJ, Casipit,CL, Doyonnas,R, Pomerantz,JH, Blau,HM. A system for quantifying dynamic protein interactions defines a role for Herceptin in modulating ErbB2 interactions. Proc Natl Acad Sci U S A 2006; 103: 1906319068.

138 Eichhorn PJ, Gili,M, Scaltriti,M, Serra,V, Guzman, M, Nijkamp,W et al. Phosphatidylinositol 3-kinase hyperactivation results in lapatinib resistance that is reversed by the mTOR/phosphatidylinositol 3-kinase inhibitor NVP-BEZ235. Cancer Res 2008; 68: 9221-9230.

139 Moody SE, Schinzel,AC, Singh,S, Izzo,F, Strickland,MR, Luo,L et al. PRKACA mediates resistance to HER2-targeted therapy in breast cancer cells and restores anti-apoptotic signaling. Oncogene 2014; 0.

140 Lang JY, Hsu,JL, Meric-Bernstam,F, Chang,CJ, Wang,Q, Bao,Y et al. BikDD eliminates breast cancer initiating cells and synergizes with lapatinib for breast cancer treatment. Cancer Cell 2011; 20: 341-356.

141 Milella M, Trisciuoglio,D, Bruno,T, Ciuffreda,L, Mottolese,M, Cianciulli,A et al. Trastuzumab down-regulates Bcl-2 expression and potentiates apoptosis induction by $\mathrm{Bcl}-2 / \mathrm{Bcl}-\mathrm{XL}$ bispecific antisense oligonucleotides in HER-2 gene--amplified breast cancer cells. Clin Cancer Res 2004; 10: 7747-7756.

142 Tanizaki J, Okamoto,I, Fumita,S, Okamoto,W, Nishio,K, Nakagawa,K. Roles of BIM induction and survivin downregulation in lapatinib-induced apoptosis in breast cancer cells with HER2 amplification. Oncogene 2011; 30: 4097-4106. 
143 Cruickshanks N, Hamed,HA, Bareford,MD, Poklepovic,A, Fisher,PB, Grant,S et al. Lapatinib and obatoclax kill tumor cells through blockade of ERBB1/3/4 and through inhibition of BCL-XL and MCL-1. Mol Pharmacol 2012; 81: 748-758.

144 Testa U. Apoptotic mechanisms in the control of erythropoiesis. Leukemia 2004; 18: 1176-1199.

145 Yip KW, Reed,JC. Bcl-2 family proteins and cancer. Oncogene 2008; 27: 6398-6406.

146 Cory S, Adams,JM. The Bcl2 family: regulators of the cellular life-or-death switch. Nat Rev Cancer 2002; 2: 647-656.

147 Valabrega G, Capellero,S, Cavalloni,G, Zaccarello,G, Petrelli,A, Migliardi,G et al. HER2-positive breast cancer cells resistant to trastuzumab and lapatinib lose reliance upon HER2 and are sensitive to the multitargeted kinase inhibitor sorafenib. Breast Cancer Res Treat 2011; 130: 29-40.

148 Xia W, Bacus,S, Hegde,P, Husain,I, Strum,J, Liu,L et al. A model of acquired autoresistance to a potent ErbB2 tyrosine kinase inhibitor and a therapeutic strategy to prevent its onset in breast cancer. Proc Natl Acad Sci U S A 2006; 103: 7795-7800.

149 Martin AP, Mitchell,C, Rahmani,M, Nephew,KP, Grant,S, Dent,P. Inhibition of MCL-1 enhances lapatinib toxicity and overcomes lapatinib resistance via BAKdependent autophagy. Cancer Biol Ther 2009; 8: 2084-2096. 


\section{Chapter 2}

\section{Regulation of anti-apoptotic signaling by Kruppel-like factors 4 and 5 mediates lapatinib resistance in breast cancer}

Mark K. Farrugia ${ }^{1,2}$, Sriganesh B. Sharma ${ }^{1,2}$, Chen-Chung Lin $^{1,3}$, Sarah L. McLaughlin Daniel B. Vanderbilt ${ }^{1,2}$, Amanda G. Ammer ${ }^{3}$, Mohamad A. Salkeni ${ }^{3,4}$, Peter Stoilov ${ }^{1,2,3}$, Yehenew M. Agazie ${ }^{1,2,3}$, Chad J. Creighton ${ }^{5}$ and J. Michael Ruppert ${ }^{1,2,3, \#}$

${ }^{1}$ Department of Biochemistry, West Virginia University, Morgantown, West Virginia 26506, USA

${ }^{2}$ Program in Cancer Cell Biology, West Virginia University, Morgantown, West Virginia 26506, USA

${ }^{3}$ The Mary Babb Randolph Cancer Center, West Virginia University, Morgantown, West Virginia 26506, USA

${ }^{4}$ Department of Medicine, West Virginia University, Morgantown, West Virginia 26506, USA

${ }^{5}$ Department of Medicine and Dan L. Duncan Cancer Center, Division of Biostatistics, Baylor College of Medicine, Houston, TX 77030, USA

${ }^{\#}$ Corresponding Author: Department of Biochemistry, P.O. Box 9142, West Virginia University Health Sciences Center, 1 Medical Center Dr., Morgantown, WV 26506. Phone: (304) 293-5246. Fax: (304) 293-4667. E-mail: mruppert@hsc.wvu.edu.

Running title: KLF4 and KLF5 mediate lapatinib resistanc

This Chapter has been published in Cell Death and Disease, 2015 Mar 19;6:e1699. doi: 10.1038/cddis.2015.65 


\section{Abstract}

The Kruppel-like transcription factors (KLFs) 4 and 5 (KLF4/5) are coexpressed in mouse embryonic stem cells, where they function redundantly to maintain pluripotency. In mammary carcinoma KLF4/5 can each impact the malignant phenotype, but potential linkages to drug resistance remain unclear. In primary human breast cancers we observed a positive correlation between KLF4/5 transcript abundance, particularly in the HER2-enriched subtype. Furthermore, KLF4/5 protein was rapidly upregulated in human breast cancer cells following treatment with the HER2/EGFR inhibitor, lapatinib. In addition, we observed a positive correlation between these factors in the primary tumors of genetically engineered mouse models (GEMMs). In particular the levels of both factors were enriched in the basal-like tumors of the C3(1) TAg GEMM. Using tumor cells derived from this model as well as human breast cancer cells, suppression of KLF4 and/or KLF5 sensitized HER2-overexpressing cells to lapatinib. Indicating cooperativity, greater effects were observed when both genes were depleted. KLF4/5-deficient cells had reduced basal mRNA and protein levels of the anti-apoptotic factors MCL1 and BCLXL. Moreover, MCL1 was upregulated by lapatinib in a KLF4/5-dependent fashion, and enforced expression of MCL1 in KLF4/5-deficient cells restored drug resistance. Additionally, combined suppression of KLF4/5 in cultured tumor cells additively inhibited anchorageindependent growth, resistance to anoikis, and tumor formation in immunocompromised mice. Consistent with their cooperative role in drug resistance and other malignant properties, KLF4/5 levels selectively stratified human HER2-enriched breast cancer by distant metastasis- free survival. These results identify KLF4 and KLF5 as cooperating protumorigenic factors and critical participants in resistance to lapatinib, furthering the rationale for combining antiMCL1/BCL-XL inhibitors with conventional HER2-targeted therapies. 
Keywords: KLF4, KLF5, lapatinib, HER2, MCL1, BCL-XL

Abbreviations: BCL2, B-Cell lymphoma 2; BCL-XL, B-Cell lymphoma-extra large; C3(1) TAg, SV40 Large T Antigen transgenic mice under control of the C3(1)/prostatein promoter; ChIP-seq, chromatin immunoprecipitation combined with high throughput sequence; CI, Confidence interval; DAPI, 4',6-diamidino-2-phenylindole; DFS, disease free survival; DMFS, distant metastasis-free survival; ES cells, embryonic stem cells; EGFR, epidermal growth factor receptor; GEMM, genetically engineered mouse model; HER2, human epidermal growth factor receptor 2; KLF, Kruppel-like factor; KLF4/5, KLF4 and KLF5; MAPK, Mitogen-activated protein kinase; miR-206, MicroRNA-206; MMI, Mitochondrial membrane integrity; MMTV, mouse mammary tumor virus promoter; MCL1, Myeloid leukemia cell 1; PI3K, phosphatidylinositide 3-kinase; qRT-PCR, Quantitative reverse transcription and polymerase chain reaction; RTK, Receptor tyrosine kinase; TCGA, The Cancer Genome Atlas; TNBC, triple negative breast cancer 


\section{Introduction}

In mouse embryonic stem (ES) cells, pluripotency is maintained by the redundant function of three Kruppel-like transcription factors, KLF2, KLF4, and KLF5. ${ }^{1}$ Furthermore, as determined by chromatin immunoprecipitation combined with high throughput sequence analysis (ChIP-seq), KLF4 and KLF5 (KLF4/5) have both overlapping and distinct target genes. ${ }^{2}$ Depletion of Klf4 or Klf5 in the anterior eye elicits similar developmental phenotypes, while in other tissues they exert opposing influences. ${ }^{3-5}$ For example, KLF4/5 differentially affect the expression of several cell cycle regulatory proteins, such as CCND1, CCNB1 and p21 ${ }^{\text {Waf1/Cip1 } 3 .}$ In adult tissues KLF4 and/or KLF5 are induced by a variety of stress stimuli and can promote cell survival in diverse contexts. ${ }^{3,6-9}$

In breast cancer, KLF4/5 protein levels or mRNA abundance are elevated in aggressive primary breast carcinomas. ${ }^{10-13}$ Consistent with these results, promoter demethylation of KLF4 or KLF5 in breast tumors is associated with an unfavorable clinical course. ${ }^{14}$ Individually, both KLF4/5 exert oncogenic functions in experimental models of cancer such as cellular transformation, migration, invasion, and xenograft formation. ${ }^{15-19}$ Although signaling mechanisms remain to be elucidated, KLF4 directly regulates the transcription of microRNA-206 (miR-206) to promote tumor cell survival and tumor initiation in athymic mice (manuscript submitted). ${ }^{18,20}$ While independently KLF4/5 have important roles in breast cancer, the relationship between the two genes in this disease remains understudied.

We observed a positive correlation of KLF4/5 expression in the human HER2-enriched breast cancer subtype. Additionally, in these patients the median expression of both KLF4/5 significantly stratified the distant metastasis-free survival (DMFS). Clinically-approved HER2- 
targeted therapies such as lapatinib and trastuzumab (Herceptin) have significantly improved the disease free survival of patients with HER2-amplified breast cancers. ${ }^{21,22}$ However, eventual resistance to these therapies is observed in the majority of cases, representing a major obstacle to long term cures. ${ }^{23-26}$

Several mechanisms of resistance have been described, often involving sustained signaling through dimerization with other receptor tyrosine kinases (RTKs) or activating mutations in downstream effectors, namely RAS pathway components. ${ }^{27-32}$ Although numerous pathways to resistance have been characterized, it is unclear which of these mechanisms predominate in patients and how they are specifically regulated.

Interestingly, neutralization of apoptotic signaling contributes to anti-HER2 therapeutic failure. $^{33-35}$ For example, phosphorylation of BAD or overexpression of BCL-XL reduces the efficacy of these drugs. One such drug is lapatinib, a HER2/EGFR inhibitor that has activity not only in the HER2+ cancers of patients, but also shows efficacy in combination with other agents in basal-like breast cancer models. ${ }^{36,37}$ We observed that lapatinib treatment of HER2-amplified tumor cells resulted in the rapid induction of KLF4/5. Subsequent experiments demonstrated a novel role for the endogenous KLFs in the regulation of anti-apoptotic factors. As shown by shRNA studies, in the presence of lapatinib KLF4/5 co-regulated the expression of MCL1 and cooperated to confer lapatinib resistance. Even in the absence of drug treatment, the endogenous KLFs were critical for maintaining basal levels of the anti-apoptotic factors MCL1 and BCL-XL, and collaboratively promoted the malignant phenotype. KLF4/5 were positively correlated with MCL1 in primary breast tumors, and enforced expression of MCL1 was sufficient to rescue the lapatinib sensitivity of KLF4/5-deficient cells. These results identify KLF4/5 as inducible regulators of lapatinib resistance through mediation of anti-apoptotic signaling. 


\section{Results}

\section{KIf4 and KIf5 are differentially expressed and positively correlated in genetically engineered mouse models (GEMMs) of breast cancer}

To better understand how the expression of these two KLFs are altered during mammary tumorigenesis, we analyzed the levels of Klf4/5 in GEMMs of breast cancer. ${ }^{38}$ As a complement to human tumor analysis, individual GEMMs offer a genetically homogenous background where tumors arise in the context of specific genetic alterations.

Of 108 tumors in the microarray dataset, we analyzed 58 tumor samples across 9 different GEMMs. ${ }^{38}$ We omitted models that had very low abundance of Klf4/5, including p53deficient models and models on the BALB/c background. We also omitted samples that did not cluster into an intrinsic subtype (14 tumor samples), and we excluded the mesenchymal subgroup tumors due to heterogeneity in GEMM of origin (5 tumors) (Fig. 1A). Unlike for Klf5, Klf4 expression as determined by microarray analysis varied substantially across the mouse model tumors, with higher expression in mice transgenic for the coding region of SV40 Large T antigen driven by the C3(1)/prostatein promoter (i.e., C3(1) TAg) than in MMTV-Neu tumors. Collectively, KIf4 levels were significantly lower in GEMMs that generated predominantly luminal tumors comparative to tumors with basal characteristics $(\mathrm{p}<0.0001)$.

Interestingly, the expression of Klf4/5 were positively correlated in the 58 GEMM tumors $(\mathrm{R}=0.5658, \mathrm{p}<0.0001)$ (Fig. 1B, upper panel). Among the tumor subgroups, the strongest correlation was observed in basal tumors $(\mathrm{R}=0.8242, \mathrm{p}=0.0005)$ (Fig. 1B, lower panel). Significant correlations were also present in the luminal tumors (data not shown). Indicating specificity, Klf4 or Klf5 did not correlate or trend toward a correlation with another KLF, Klf2 (data not shown). ${ }^{1}$ 
To more accurately quantitate gene expression, we isolated spontaneous tumors from the C3(1) TAg and MMTV-Neu models and measured mRNA levels by qRT-PCR (Fig. 1C, left panel). In the C3(1) TAg tumors Klf4/5 abundance averaged 2.6- and 3.5-fold higher than normal breast, respectively. In contrast, MMTV-Neu tumors showed dramatically reduced levels of both factors. The two models differed in average abundance by 158- and 26-fold for Klf4/5, respectively $(\mathrm{p}<0.0001)$. In these tumors Klf4/5 mRNA correlated strongly (Fig. 1C, right panel; $\mathrm{R}=0.8273$, $\mathrm{p}=0.0024)$. Consistent with animal model data, the protein expression of KLF4/5 in 10 different human breast cancer cell lines was positively correlated $(\mathrm{R}=0.8847, \mathrm{p}=0.0007)$ (Fig. 1D). For KLF5, the smaller fragment of approximately $48 \mathrm{kDa}$ present in the luminal breast cancer cell lines may be attributed to estrogen-dependent processing. ${ }^{39}$

\section{Prognostic significance of KLF4 and KLF5 in HER2-enriched breast cancer}

As KLF4/5 were correlated in breast cancer models, we next examined whether this relationship is informative to patient outcome. We performed Kaplan-Meier analyses of DMFS using a previously described compendium of gene expression profiling datasets representing 1065 cases. ${ }^{40}$ Median expression levels were used to define the groups. We excluded normal breastlike tumors from analysis because these samples often contain high amounts of contaminating normal cells. ${ }^{41}$

Across all tumors KLF4 levels showed no significant relationship with DMFS (Fig. 2A). In contrast, elevated KLF5 was associated with shortened DMFS, consistent with prior observations (hazard ratio [HR], 1.3; 95\% confidence interval (CI), 1.1-1.7; p=0.01) (Fig. 2A). ${ }^{11}$

Interestingly, analysis of DMFS in each intrinsic subtype revealed a trend for increased KLF5 expression and poor outcome in the HER2-enriched tumors (HR, 1.6; CI, 1.0-2.5, nominal 
$\mathrm{p}=0.05$ ) (Fig. 2B). Furthermore, KLF4 expression showed a similar trend in this context (HR, 1.4; CI, 0.9-2.2, $\mathrm{p}=0.13$ ). No such relationships were observed in a similarly high risk group, basal-like/claudin-low (Fig. 2B), nor in the luminal A or luminal B groups (Supplemental Fig. 1). Furthermore, the expression of $K L F 4 / 5$ together (i.e., $<$ median levels of each gene vs $\geq$ median levels of each gene) considerably stratified DMFS in the HER2-enriched subtype (HR, 2.4; CI, 1.2-4.5, $\mathrm{p}=0.011$ ) (Fig. 2C). HER2-enriched tumors with elevated KLF4/5 levels had DMFS similar to $43 \%$ at 15 years post-diagnosis. This effect was unique to the HER2-enriched subtype, as no other group showed any trend (Fig. 2C and Supplemental Fig. 1). To assess survival in patients with elevated expression of only one of the two KLFs, we analyzed the four survival curves defined by 2x2 contingency using the median scores for KLF4/5 (Supplemental Fig. 1). The log rank test for trend identified a significant trend between KLF4/5 expression and median DMFS $(\mathrm{p}=0.016)$.

\section{Endogenous KLF4 and KLF5 are induced by lapatinib in breast cancer}

We next examined the transcript abundance of $K L F 4 / 5$ as determined by RNAseq analysis of patient breast tumors via The Cancer Genome Atlas (TCGA) Research Network. Among the breast cancer intrinsic subtypes, we observed the expression of the two factors to be most highly correlated in HER2-enriched tumors (Fig. 3A and Supplemental Fig. 2). Given that KLF4/5 appeared to represent positively correlated prognostic factors in the HER2-enriched breast cancer subtype, we subsequently investigated the interdependence of KLF4/5 expression with exposure to, or resistance to, HER2-targeted therapy.

Interestingly, lapatinib promoted the expression of both the KLF4 and KLF5 proteins (Fig. 3B). Trastuzumab treatment yielded similar results (Fig. 3C). In these experiments 
phosphorylated AKT levels served as a positive control for HER2 inhibition (Fig. 3B and 3C). These effects appeared to be transcriptionally independent, as the respective mRNA levels were not significantly altered (Fig. 3D). Nor did enhanced efficiency of protein translation appear to account for upregulation of KLF4. Rather, the translational efficiency of the full-length KLF4 transcript, as determined using a previously described translation reporter, pMIR-Report-LucKLF4-FL, was actually decreased by lapatinib treatment (Fig. 3E, left panel). ${ }^{18}$ This decrease is expected when KLF4 transcriptional activity is elevated, attributed to a well-characterized negative feedback signal by which KLF4 induces miR-206 and suppresses its own translation. ${ }^{18}$ Indeed, in lapatinib-treated cells the elevated KLF4 was associated with increased levels of miR206, which can then directly target the KLF43' UTR (Fig. 3E, right panel). ${ }^{18,20}$

As neither KLF4 transcription nor its translational efficiency appear to be upregulated by lapatinib, the results are consistent with lapatinib-mediated stabilization of the KLF4 protein. Therefore lapatinib may function similarly to serum starvation to mediate a prolonged KLF4 half-life. ${ }^{42}$ Several experiments to directly assess half-life were unsuccessful due to the combined toxicity when cells were exposed to both lapatinib and the protein synthesis inhibitor cycloheximide. Nevertheless, the results indicate that lapatinib treatment of HER2-positive breast cancer cells can enhance KLF4 protein expression and its transcriptional activity as indicated by miR-206 levels.

\section{Endogenous KLF4 and KLF5 mediate lapatinib resistance in breast cancer}

Based on these results we hypothesized that KLF4/5 are functionally important in the response to lapatinib. We therefore depleted KLF4/5 in HER2-amplified human BT474 and mouse M6 breast cancer cells, using distinct shRNA hairpins for each of the human and mouse genes (Fig. 
3F, left panels). M6 cells are a HER2-overexpressing mammary cancer cell line derived from a basal-like GEMM that is enriched for KIf4/5, the C3(1) TAg model. ${ }^{43}$ Unlike many basal-like models, M6 cells overexpress both Egfr and Erbb2, the two RTKs targeted by lapatinib. Compared to the nontargeting control, single knockdown of KLF4 or KLF5 in the BT474 and M6 models significantly sensitized the cells to lapatinib treatment (Fig. 3F, right panels). Moreover, coreduction of both KLF4/5 further sensitized the cells to lapatinib, indicating cooperativity. In these experiments suppression of KLF5 led to a subtle reduction of KLF4, suggesting the possibility of crosstalk between the two factors (Fig. 3F, left panels). In agreement with the knockdown studies, ectopic expression of KLF4/5 in M6 enhanced resistance to lapatinib treatment (Fig. 3G)

To further characterize their role in drug resistance, we assessed mitochondrial membrane integrity (MMI) in these cells following lapatinib exposure (Fig. 3H). Disruption of MMI is a key step of the intrinsic pathway of apoptosis. Following 24 hours of lapatinib treatment, the single knockdown cell lines had reduced MMI compared to the controls and suppression of both KLF4/5 produced an additive effect. Supporting an impact of KLF4/5 on the intrinsic pathway of apoptosis, reduction of KLF4/5 enhanced cleaved caspase-9 following exposure to lapatinib, with additive effects when both KLFs were depleted (Fig. 3I).

\section{KLF4 and KLF5 cooperate to promote malignant properties}

We next determined whether KLF4/5 contributed to malignant properties independently of lapatinib exposure. Relative to the control, depletion of either KLF4 or KLF5 in M6 cells significantly impacted anchorage-independent growth, as indicated by reduced colony forming ability (Fig. 4A). Furthermore, there was an additive reduction of colony number following codepletion. We similarly observed cooperativity in a gain-of-function context, as ectopic 
expression of KLF4 and KLF5 enhanced colony forming ability in immortalized human breast epithelial cells (HMLE, Fig. 4B).

Expanding on this observation, we evaluated the ability of these factors to impact tumor formation in athymic mice. Individual reduction of each KLF significantly reduced xenograft growth, with additive effects in the double knockdown cells (Fig. 4C, left panel). Comparable results on tumor formation were obtained using an independent shRNA to target each factor (Fig. 4C, right panel).

To determine whether these results reflect deficiencies in prosurvival signaling, we examined whether KLF4/5 could cooperatively influence cell death following matrix deprivation (anoikis). Single knockdown of KLF4 or KLF5 sensitized cells to anoikis as determined by trypan blue exclusion, with cooperative effects following codepletion (Fig. 4D, left panel). Conversely, overexpression of these factors in HMLE enhanced anoikis resistance (Fig. 4D, right panel). Determination of cell death by an independent method yielded similar results (Fig. 4E).

\section{KLF4 and KLF5 depletion is associated with reduced expression of anti-apoptotic BCL2 family members}

As KLF4/5-depleted cells consistently exhibited defects in cell survival, we analyzed molecular effectors of this phenotype. The intrinsic pathway of apoptosis is critical in both lapatinib resistance and anoikis. ${ }^{44}$ We therefore examined the association of KLFs with factors known to participate in apoptotic signaling.

In response to lapatinib or trastuzumab treatment we observed induction of not only KLF4/5 (Fig. 3B and 3D), but also the anti-apoptotic BCL2 members BCL2, BCL-XL (BCL2L1), and MCL1 (Fig. 5A). While BCL2 was increased in response to lapatinib, BCL2 
levels were decreased upon trastuzumab exposure, consistent with previous studies. ${ }^{45}$ Despite the well-documented oncogenic role for BCL2 in hematological malignancies, its expression is correlated with a favorable patient outcome in breast cancer. ${ }^{46}$

Single knockdown of either KLF4 or KLF5 greatly reduced BCL-XL levels in untreated M6 and BT474 cells (Fig. 5B and Fig. 5C, left panel). Interestingly, human KPL4 cells required depletion of both KLFs to impact BCL-XL abundance, possibly attributed to the activating PI3K mutation present in this HER2-amplified, inflammatory breast cancer cell line (Fig. 5B) ${ }^{47}$

In both BT474 and KPL4 cells, a reduction of the MCL1 protein level was evident when KLF4/5 were cosuppressed (Fig. 5B). Due to lack of a suitable antibody, we could not reliably detect murine MCL1 (Fig. 5C, left panel). Regardless, in these cells the KLFs cooperated to maintain Mcl1 and Bcl-xl transcript levels, as shown by qRT-PCR (Fig. 5C, right panel). Ectopic expression of KLF4/KLF5 in HMLE cells cooperated to increase MCL1 expression, however BCL-XL levels responded primarily to KLF4 (Fig. 5D).

Although the KLFs impacted BCL2 levels in M6 cells, this regulation was not apparent in BT474 cells (Figs. 5B and C). qRT-PCR transcript analysis of other BCL2 family members including Bad, Bax, and Bid revealed no significant effects by modulation of the two KLFs (data not shown).

To extend these in vitro studies, we assessed the co-presence of KLF4/5 expression with $M C L 1, B C L-X L$, and/or BCL2 in human tumors (Table 1) ${ }^{48,49}$ Using a $+/-1.5$ z-score range to define high and low expression groups, we evaluated the mutual exclusivity/inclusivity in 958 human breast tumors that were analyzed by microarray. Both KLF4/5 co-occurred with MCL1. In addition, $K L F 4$ and $B C L-X L$ expression were mutually inclusive, as were $K L F 5$ and BCL2. In 
agreement with our previous observations (Fig. 3A), KLF4/5 expression co-occured in these tumors.

To more directly test for a correlation in patient samples we utilized the RNAseq database generated by TCGA. Across 890 breast tumors, the transcript abundance for KLF4, KLF5 and MCL1 was positively correlated (Fig. 5E). Using this dataset we observed no significant positive correlations between KLFs and the other anti-apoptotic genes, $B C L-X L$ and BCL2 (data not shown). Despite these results, extensive ChIP studies in BT474 cells that analyzed 5 kilobase pairs upstream of the transcriptional start site, the body of the gene, and 3 kilobase pairs downstream, failed to identify any KLF4/5 association with the mouse or human MCL1 loci (see Discussion).

We next examined whether KLF4/5 expression was required for the induction of antiapoptotic molecules by lapatinib. We focused on MCL1, as this molecule displayed a robust induction following HER2-inhibition and exhibited consistent relationships with the KLFs in human tumors (Table 1, Figs. 5A and E). To ensure a suitable number of viable, lapatinibsensitive KLF-depleted cells for analysis we used a reduced lapatinib concentration of $250 \mathrm{nM}$. While individual reduction of KLF4 or KLF5 did not substantially impact the lapatinib-mediated induction of MCL1 (data not shown), co-suppression of both KLFs blunted this response (Fig. $5 \mathrm{~F})$.

To validate the importance of MCL1 in lapatinib resistance, we reduced MCL1 levels in BT474 cells using siRNA smart pool (Fig. 5G). Compared to the non-targeting control, siMCL1 cells demonstrated increased sensitivity to lapatinib treatment. Similarly, a small molecule MCL1 inhibitor, UMI-77, yielded comparable results to the siRNA studies (data not shown). 
Conversely, MCL1 overexpression in KLF4/5 knockdown BT474 cells was sufficient to restore lapatinib resistance (Fig. 5H).

\section{Discussion}

Targeted therapies have significantly improved the disease-free survival of breast cancer patients, including patients with ER+ or HER2+ breast cancers, and these therapies hold promise for triple-negative breast cancer (TNBC), particularly when used in combination. ${ }^{36,37}$ For HER2+ breast cancer, clinically-approved therapies include the monoclonal antibodies trastuzumab and pertuzumab and, for third or fourth line therapy of metastatic disease, the small molecule lapatinib.

For intrinsically aggressive breast cancers such as HER2-enriched tumors, therapeutic resistance is common, as evidenced by the progression of unresponsive tumors. ${ }^{23-26}$ Similar to lapatinib and trastuzumab, it is likely that resistance mechanisms will impact the newer classes of HER2/EGFR inhibitors, such as neratinib. ${ }^{50}$ Therefore, a better understanding of the molecular drivers of resistance could enable the identification of effective drug combinations to improve clinical efficacy.

Many studies support shared resistance mechanisms between lapatinib and trastuzumab. ${ }^{27-32,35}$ These include the sustained activation of downstream effectors of HER2 including phosphatidylinositide 3-kinase (PI3K), mTOR, and mitogen-activated protein kinase (MAPK). Through its regulation by these pathways or by other signaling entities, the intrinsic cell death pathway confers resistance to anti-HER2 treatments. ${ }^{33-35,45,51}$ Thus, the therapeutic effect of anti-HER2 treatments can likely be modulated using small molecule inhibitors or genetic methods to perturb the balance between pro-apoptotic and anti-apoptotic signaling. For 
example, a constitutively active pro-apoptotic Bik enhances lapatinib-mediated apoptosis. ${ }^{33}$ Likewise, knockdown or overexpression of either MCL1, BCL2, or BCL-XL significantly alters the efficacy of lapatinib in HER2-amplified cells. ${ }^{33,35}$

In our study the KLFs mediated lapatinib resistance in a cooperative fashion, a result obtained in HER2-amplified BT474 cells and in M6 cells, a HER2 over-expressing cell line. ${ }^{38,52}$ Likely contributing to this effect, the two KLFs independently impacted basal levels of the antiapoptotic factors MCL1 and BCL-XL in drug-naïve cells. Furthermore, in lapatinib-treated BT474 cells, we were surprised to observe upregulation of BCL-XL and MCL1 upon HER2 inhibition. The induction of MCL1 required the activity of KLF4/5, as indicated by analysis of the KLF4/5 deficient cells. We are currently assessing other tumor models for KLF4/5dependent upregulation of anti-apoptotic factors in response to small molecule inhibitors.

There are only a few established links between the KLFs, anti-apoptotic BCL2 family proteins, and HER2. ${ }^{53-55}$ In the current study, we observed co-expression of KLF4/5 and MCL1 in human breast tumors and breast cancer models. Also, in mouse embryonic stem cells the Mcl1 locus was significantly enriched via ChIP-seq using antibodies to either KLF4 or KLF5. ${ }^{2,56}$ Despite these results, and the clear dependence of MCL1 on KLF4/5 expression (Fig. 5B and 5C), the MCL1 locus was not enriched in KLF4/5 ChIP assays, in which MIR206 served as a positive control (data not shown). ${ }^{20}$ These results suggest an indirect relationship, although the possibility of direct regulation through a distal binding site cannot be excluded.

We found that KLF4/5 mRNA levels were correlated most strongly in the HER2-enriched tumors, and these factors were selectively prognostic within this subtype. Previous studies have linked adverse clinical outcome to increased nuclear localization of KLF4, to elevated KLF5 mRNA, and to promoter demethylation of KLF4 or KLF5. ${ }^{10-14}$ In our study, we observed 
shortened DMFS for patients with HER2-enriched tumors containing elevated levels of both KLF4 and KLF5, implicating these two KLFs as novel prognostic factors (Fig. 2D).

In any single breast tumor subtype or in all tumors combined, KLF4 on its own had little effect (Fig. 2B). In contrast to these results, a previous study associated elevated KLF4 transcript levels with prolonged disease-free survival (DFS). ${ }^{57}$ As overall survival, DFS, and DMFS correlate strongly in breast cancer, the utilization of these different endpoints seems unlikely to account for the distinct results. ${ }^{58-60}$ Instead, the discrepancy may be attributed to the analysis of different patient populations, to differences in sample size, or to methodological differences in sample processing.

The current study identifies a novel drug-resistance program composed of KLF4/5, with likely origins in the stress response signaling of KLFs in normal cells. In response to deficient RTK signaling, KLF4/5 can coordinate a prosurvival response that includes BCL2 family proteins, miR-206, and likely other factors. Future studies will examine the predictive utility of KLF4/5 for guiding patient therapy, for example in patients with HER2+ tumors. Taken together, the identification of KLF4/5 as intermediaries between HER2 and the BCL2 family members significantly strengthens the rationale for combined therapeutic inhibition of HER2 and BCLXL/MCL1 to combat drug resistance.

\section{Materials and Methods}

\section{Cell lines and tissue culture}

M6 cells were obtained from Jeffrey E. Green (National Cancer Institute), KPL4 cells were from Afsaneh Keyhani (MD Anderson) and HMLE cells were from Robert A.Weinberg (Whitehead Institute). Cells were cultured as previously described.$^{52,61,62}$ T47D, BT-20, ZR-75-1, MCF7, 
HCC1937, BT-474, and HCC1143 cell lines were purchased from ATCC and cultured in RPMI 1640. MDA-MB-453 cells were cultured in DMEM, while MDA-MB-361 and MDA-MB-468 were cultured in DMEM/Ham's F12 50:50. All were supplemented with 10\% (v/v) FBS (Hyclone, GE Healthcare Life Sciences, Logan, UT), penicillin, and streptomycin. Lapatinib (Selleck, Boston , MA) was dissolved in DMSO and used at the indicated concentrations. Trastuzumab (Herceptin) was obtained from the Mary Babb Randolph Cancer Center (Morgantown,WV).

\section{Transfections and retroviral transduction}

The control shRNA vector (nonsilencing-GIPZ lentiviral shRNA control [shCtrl]; RHS4346) and the shRNA vectors for murine Klf4 (V3LMM_459916, V3LMM_524009), murine Klf5 (V3LMM_489119, V2LMM_73715) and human KLF5 (V2LHS_150118 and V2LHS_150120) were purchased from Open Biosystems (Pittsburgh, PA). shRNAs targeting human KLF4 have been previously described. ${ }^{20}$

siMCL1 ON-TARGETplus smart pool was purchased from Dharmacon (Lafayette, CO) and transfected using Lipofectamine RNAiMAX Reagent, Life Technologies (Grand Island, $\mathrm{NY}$ ), per the manufacturer's recommendations.

Luciferase-based reporter assay of translational efficiency was performed as previously reported, using pMIR-Report-Luc-KLF4-FL and with normalization to pRL-TK. ${ }^{18}$

KLF4 and KLF5 were expressed in human cells using the lentiviral vector, pLuT7. KLF4 was ectopically expressed in M6 cells using pBabe-puro-KLF4.$^{18}$ pBabe-puro MCL-1 was a gift from Roger Davis (Addgene plasmid \# 25371). ${ }^{63}$ Vectors were packaged into viral particles as 
previously described. ${ }^{18}$ Cells were infected with viral supernatant supplemented with $10 \mu \mathrm{g} / \mathrm{mL}$ polybrene, centrifuged at $2500 \mathrm{rpm}$ for 1.5 hours at $30^{\circ} \mathrm{C}$, and selected in puromycin $(1.0 \mu \mathrm{gg} / \mathrm{ml})$.

\section{Immunoblot analysis}

Cell lysis, gel electrophoresis, transfer, and immunoblot analysis was performed as described. ${ }^{18}$ Primary antibodies were KLF4 (H180, Santa Cruz, Dallas, TX), KLF5 (Millipore, Billerica, MA), MCL1 (S19, Santa Cruz), BCL-XL (L19, Santa Cruz), BCL2 (clone 7/Bcl-2, BD Biosciences, San Jose, CA), phospho-AKT(Ser473, Cell Signaling, Danvers, MA), AKT (Cell Signaling), Caspase-9 (Cell Signaling, 9502), and $\beta$-Actin (C-4, Sigma, St. Louis, MO).

\section{Animal studies}

C3(1) TAg mice and MMTV-Neu mice (202Mul) were obtained from Jax Labs (Bar Harbor, ME). Female athymic nude mice (Charles River, Frederick, MD) were obtained at 6-8 weeks of age. $2.0 \times 10^{6}$ cells in were injected into the $4^{\text {th }}$ mammary fat pad in $100 \mu \mathrm{l}$ of DMEM. Caliper measurements were performed twice per week to measure tumor volume. Tumor volume was calculated according to $\pi\left(\mathrm{L} 1 \times \mathrm{L}_{2}^{2}\right) / 6$ (L1, long axis; L2, short axis). All animal procedures were performed under an approved ACUC protocol.

\section{Quantitative, real-time, reverse transcription and PCR (qRT-PCR) analysis of mRNA}

Mouse breast tumors or normal mammary glands were harvested from euthanized mice and snap frozen. Tissue was mechanically dissociated using glass beads and total RNA was isolated as previously described. ${ }^{64}$ For cell lines, total RNA was extracted using the RNeasy minikit (Qiagen, Valencia, CA). qRT-PCR was performed as previously described. ${ }^{18}$ Primers used for qRT-PCR can be found in Supplemental Table 1. Of the housekeeping genes analyzed using RNA from normal mouse mammary gland and tumors, including Rplp0, Gapdh, and B2m, the 
Rplp0 levels showed the best correlation with total RNA quantity. Gene expression assays were therefore normalized using this transcript. ${ }^{65}$

\section{Gene expression analysis of human breast tumors}

Data generated using the UNC Illumina HiSeq RNAseqV2 platform were downloaded from TCGA (http:/cancergenome.nih.gov/, March 11, 2013). Statistical programming software R (version 3.0.1) was used to assemble and process the data. Molecular subtyping was

accomplished using the Bioconductor 2.12 genefu R package. ${ }^{66}$ At the time of this study, patient follow-up data for TCGA samples were not sufficiently mature for survival analysis. For survival analyses, the compendium of gene expression array datasets of breast cancer was previously compiled, with subtypes assigned as described elsewhere. ${ }^{40,67}$ Probes used for $K L F 4 / 5$ were 221841_s_at and 209211_at, respectively.

\section{Luminescence based cell viability assay}

$2 \times 10^{3}$ cells/well (BT474) or $5 \times 10^{2}$ cells/well (M6) were transferred to 96-well plates and cultured for 96 hours in the indicated concentrations of lapatinib. Five replicates were used for each concentration of drug. Fresh media and lapatinib was added to the cells after 48 hours. Viable cell number was determined via the ATPlite Luminescence ATP Detection Assay System (PerkinElmer, Waltham, MA). Relative \% viability was determined by normalizing each condition to DMSO treatment only.

\section{Anoikis sensitivity assays}

The anoikis procedure and quantitation of cell death by trypan blue exclusion was previously described. ${ }^{20}$ Alternatively, cell death was determined using fluorescent microscopic analysis (Zeiss Axio Imager Z2) of cells stained with propidium iodide (PI) and DAPI to identify dead 
cells and total cell number, respectively. ${ }^{68}$ Quantitation of PI/DAPI stained cells was performed using Image $\mathrm{J}$.

\section{Soft agar colony formation}

SeaPlaque agarose (Lonza) was dissolved in 1X PBS and autoclaved. The agar layers contained complete growth media and consisted of $1.0 \mathrm{~mL} 0.5 \%(\mathrm{w} / \mathrm{v})$ agar underlay, $2.0 \mathrm{~mL}$ of $0.5 \%$ agar cell layer containing $1.5 \times 10^{3}$ cells/mL, and $1 \mathrm{~mL}$ of $0.3 \%$ agar overlay per well of a 6 -well plate. $250 \mu \mathrm{l}$ of growth media was added onto the top layer. The plates were wrapped in parafilm and incubated at $37^{\circ} \mathrm{C}$ for 14 days, with an additional $250 \mu$ of complete growth media added after 7 days. Colonies were visualized with an Epson Perfection vV700 Photo scanner and colonies >200 microns were counted.

\section{Flow cytometry}

Following 24 hour treatment with $1 \mu \mathrm{M}$ lapatinib, $5.0 \times 10^{5} \mathrm{M} 6$ cells were washed twice with PBS, resuspended in $300 \mu \mathrm{l}$ PBS containing 250 nM Mitotracker Deep Red dye (Invitrogen/GE Healthcare), and incubated at room temperature for 30 minutes. Stained cells were centrifuged at low speed and then resuspended in PBS and analyzed in a BD FACS Calibur flow cytometer using BD CellQuest Pro software. Plots were generated using FCS Express 4 (De novo software, Glendale, CA).

\section{Statistical analysis}

Statistical analyses were performed in GraphPad Prism 6 (GraphPad Software, San Diego, CA). RNA samples were independently analyzed by qRT-PCR three times in duplicate fashion (column, mean; bars, SE). Correlations were obtained using Spearman’s correlation. Xenograft and drug sensitivity assays were analyzed by repeated measures one-way analysis of variance (ANOVA) followed by Tukey’s multiple comparison post test. Other assays including soft agar 
growth and anoikis were assessed by one-way ANOVA followed by Tukey's post test.

Differences were considered significant when two-sided analysis yielded $\mathrm{p}<0.05$.

\section{Conflicts of interest}

The authors declare no conflicts of interests

\section{Acknowledgements}

This work was supported by grants NCI RO1 CA127405 (to J.M.R.), the Jo and Ben Statler Chair in Breast Cancer Research, and the Wilmer V. and Helen B. Morley Memorial Fund at the Mary Babb Randolph Cancer Center (MBRCC). Flow cytometry experiments were performed in the West Virginia University Flow Cytometry Core Facility, which is supported by NIH equipment grant RR020866 and the Institutional Development Award (IDeA) from the National Institute of General Medical Sciences under grants P30GM103488 (CoBRE) and P20GM103434 (INBRE). Animal work and imaging experiments were performed in the West Virginia University Microscope Imaging Facility and West Virginia University Animal Models \& Imaging Facility, which was supported by the MBRCC and NIH grants P20 RR016440, P30 RR032138/GM103488, P20 RR016477, and S10 RR026378. Work performed at the Statistics and Bioinformatics Core Resources of the Dan L. Duncan Cancer, Baylor College of Medicine, was supported by NCI grant P30 CA125123. We thank William Petros for the generous gift of trastuzumab (MBRCC) and Fengju Chen (Baylor College of Medicine) for assistance with gene expression analysis. Additionally, we thank Jeffrey E. Green for C3(1) TAg mice and M6 cells, Laura F. Gibson for sharing several antibodies, Afsaneh Keyhani for the KPL4 cell line, and Robert A. Weinberg for donating HMLE cells. 


\section{Reference List}

1 Jiang J, Chan YS, Loh YH, Cai J, Tong GQ, Lim CA et al. A core Klf circuitry regulates self-renewal of embryonic stem cells. Nat Cell Biol 2008; 10: 353-360.

2 Aksoy I, Giudice V, Delahaye E, Wianny F, Aubry M, Mure M et al. Klf4 and Klf5 differentially inhibit mesoderm and endoderm differentiation in embryonic stem cells. Nat Commun 2014; 5: 3719-.

3 McConnell BB, Ghaleb AM, Nandan MO, Yang VW. The diverse functions of Kruppellike factors 4 and 5 in epithelial biology and pathobiology. Bioessays 2007; 29: 549-557.

4 Swamynathan SK, Katz JP, Kaestner KH, Ashery-Padan R, Crawford MA, Piatigorsky J. Conditional deletion of the mouse Klf4 gene results in corneal epithelial fragility, stromal edema, and loss of conjunctival goblet cells. Mol Cell Biol 2007; 27: 182194.

5 Kenchegowda D, Swamynathan S, Gupta D, Wan H, Whitsett J, Swamynathan SK. Conditional disruption of mouse Klf5 results in defective eyelids with malformed meibomian glands, abnormal cornea and loss of conjunctival goblet cells. Dev Biol 2011; 356: 5-18.

6 Pedersen TX, Leethanakul C, Patel V, Mitola D, Lund LR, Dano K et al. Laser capture microdissection-based in vivo genomic profiling of wound keratinocytes identifies similarities and differences to squamous cell carcinoma. Oncogene 2003; 22: 3964-3976.

7 Zhao Y, Hamza MS, Leong HS, Lim CB, Pan YF, Cheung E et al. Kruppel-like factor 5 modulates p53-independent apoptosis through Pim1 survival kinase in cancer cells. Oncogene 2008; 27: 1-8.

8 Li X, Liu X, Xu Y, Liu J, Xie M, Ni W et al. KLF5 promotes hypoxia-induced survival and inhibits apoptosis in non-small cell lung cancer cells via HIF-1alpha. Int $J$ Oncol 2014; 45: 1507-1514.

9 Talmasov D, Xinjun Z, Yu B, Nandan MO, Bialkowska AB, Elkarim E et al. Kruppellike factor 4 is a radioprotective factor for the intestine following gammaradiation-induced gut injury in mice. Am J Physiol Gastrointest Liver Physiol 2015; 308: G121-G138. 
10 Pandya AY, Talley LI, Frost AR, Fitzgerald TJ, Trivedi V, Chakravarthy $\mathrm{M}$ et al. Nuclear localization of KLF4 is associated with an aggressive phenotype in earlystage breast cancer. Clin Cancer Res 2004; 10: 2709-2719.

11 Tong D, Czerwenka K, Heinze G, Ryffel M, Schuster E, Witt A et al. Expression of KLF5 is a prognostic factor for disease-free survival and overall survival in patients with breast cancer. Clin Cancer Res 2006; 12: 2442-2448.

12 Ben-Porath I, Thomson MW, Carey VJ, Ge R, Bell GW, Regev A et al. An embryonic stem cell-like gene expression signature in poorly differentiated aggressive human tumors. Nat Genet 2008; 40: 499-507.

13 Chen CJ, Lin SE, Lin YM, Lin SH, Chen DR, Chen CL. Association of expression of Kruppel-like Factor 4 and Kruppel-like Factor 5 with the clinical manifestations of breast cancer. Pathol Oncol Res 2012; 18: 161-168.

14 Kamalakaran S, Varadan V, Giercksky Russnes HE, Levy D, Kendall J, Janevski A et al. DNA methylation patterns in luminal breast cancers differ from non-luminal subtypes and can identify relapse risk independent of other clinical variables. Mol Oncol 2011; 5: 77-92.

15 Rowland BD, Bernards R, Peeper DS. The KLF4 tumour suppressor is a transcriptional repressor of p53 that acts as a context-dependent oncogene. Nat Cell Biol 2005; 7: 1074-1082.

16 Zheng HQ, Zhou Z, Huang J, Chaudhury L, Dong JT, Chen C. Kruppel-like factor 5 promotes breast cell proliferation partially through upregulating the transcription of fibroblast growth factor binding protein 1. Oncogene 2009; 28: 3702-3713.

17 Yu F, Li J, Chen H, Fu J, Ray S, Huang S et al. Kruppel-like factor 4 (KLF4) is required for maintenance of breast cancer stem cells and for cell migration and invasion. Oncogene 2011; 30: 2161-2172.

18 Lin CC, Liu LZ, Addison JB, Ivanov AV, Ruppert JM. A KLF4-miRNA-206 autoregulatory feedback loop can promote or inhibit protein translation depending upon cell context. Mol Cell Biol 2011; 31: 2513-2527.

19 Zhao D, Zhi X, Zhou Z, Chen C. TAZ antagonizes the WWP1-mediated KLF5 degradation and promotes breast cell proliferation and tumorigenesis. Carcinogenesis 2012; 33: 59-67. 
20 Sharma SB, Lin CC, Farrugia MK, McLaughlin SL, Ellis EJ, Brundage KM et al. microRNAs-206 and -21 cooperate to promote RAS-ERK signaling by suppressing the translation of RASA1 and SPRED1. Mol Cell Biol 2014.

21 Cobleigh MA, Vogel CL, Tripathy D, Robert NJ, Scholl S, Fehrenbacher L et al. Multinational study of the efficacy and safety of humanized anti-HER2 monoclonal antibody in women who have HER2-overexpressing metastatic breast cancer that has progressed after chemotherapy for metastatic disease. J Clin Oncol 1999; 17: 2639-2648.

22 Vogel C, Cobleigh MA, Tripathy D, Gutheil JC, Harris LN, Fehrenbacher L et al. Firstline, single-agent Herceptin(R) (trastuzumab) in metastatic breast cancer. a preliminary report. Eur J Cancer 2001; 37 Suppl 1: 25-29.

23 Slamon DJ, Leyland-Jones B, Shak S, Fuchs H, Paton V, Bajamonde A et al. Use of chemotherapy plus a monoclonal antibody against HER2 for metastatic breast cancer that overexpresses HER2. N Engl J Med 2001; 344: 783-792.

24 Geyer CE, Forster J, Lindquist D, Chan S, Romieu CG, Pienkowski T et al. Lapatinib plus capecitabine for HER2-positive advanced breast cancer. N Engl J Med 2006; 355: 2733-2743.

25 Verma S, Miles D, Gianni L, Krop IE, Welslau M, Baselga J et al. Trastuzumab emtansine for HER2-positive advanced breast cancer. N Engl J Med 2012; 367: 1783-1791.

26 Baselga J, Cortes J, Kim SB, Im SA, Hegg R, Im YH et al. Pertuzumab plus trastuzumab plus docetaxel for metastatic breast cancer. N Engl J Med 2012; 366: 109-119.

27 Nagata Y, Lan KH, Zhou X, Tan M, Esteva FJ, Sahin AA et al. PTEN activation contributes to tumor inhibition by trastuzumab, and loss of PTEN predicts trastuzumab resistance in patients. Cancer Cell 2004; 6: 117-127.

28 Nahta R, Yuan LX, Du Y, Esteva FJ. Lapatinib induces apoptosis in trastuzumabresistant breast cancer cells: effects on insulin-like growth factor I signaling. Mol Cancer Ther 2007; 6: 667-674.

29 Berns K, Horlings HM, Hennessy BT, Madiredjo M, Hijmans EM, Beelen K et al. A functional genetic approach identifies the PI3K pathway as a major determinant of trastuzumab resistance in breast cancer. Cancer Cell 2007; 12: 395-402. 
30 Eichhorn PJ, Gili M, Scaltriti M, Serra V, Guzman M, Nijkamp W et al.

Phosphatidylinositol 3-kinase hyperactivation results in lapatinib resistance that is reversed by the mTOR/phosphatidylinositol 3-kinase inhibitor NVP-BEZ235.

Cancer Res 2008; 68: 9221-9230.

31 Esteva FJ, Guo H, Zhang S, Santa-Maria C, Stone S, Lanchbury JS et al. PTEN, PIK3CA, p-AKT, and p-p70S6K status: association with trastuzumab response and survival in patients with HER2-positive metastatic breast cancer. Am J Pathol 2010; 177: 1647-1656.

32 Dave B, Migliaccio I, Gutierrez MC, Wu MF, Chamness GC, Wong H et al. Loss of phosphatase and tensin homolog or phosphoinositol-3 kinase activation and response to trastuzumab or lapatinib in human epidermal growth factor receptor 2-overexpressing locally advanced breast cancers. J Clin Oncol 2011; 29: 166173.

33 Lang JY, Hsu JL, Meric-Bernstam F, Chang CJ, Wang Q, Bao Y et al. BikDD eliminates breast cancer initiating cells and synergizes with lapatinib for breast cancer treatment. Cancer Cell 2011; 20: 341-356.

34 Valabrega G, Capellero S, Cavalloni G, Zaccarello G, Petrelli A, Migliardi G et al. HER2-positive breast cancer cells resistant to trastuzumab and lapatinib lose reliance upon HER2 and are sensitive to the multitargeted kinase inhibitor sorafenib. Breast Cancer Res Treat 2011; 130: 29-40.

35 Moody SE, Schinzel AC, Singh S, Izzo F, Strickland MR, Luo L et al. PRKACA mediates resistance to HER2-targeted therapy in breast cancer cells and restores anti-apoptotic signaling. Oncogene 2014; 0.

36 Liu T, Yacoub R, Taliaferro-Smith LD, Sun SY, Graham TR, Dolan R et al. Combinatorial effects of lapatinib and rapamycin in triple-negative breast cancer cells. Mol Cancer Ther 2011; 10: 1460-1469.

37 Nowsheen S, Cooper T, Stanley JA, Yang ES. Synthetic lethal interactions between EGFR and PARP inhibition in human triple negative breast cancer cells. PLoS One 2012; 7: e46614-.

38 Herschkowitz JI, Simin K, Weigman VJ, Mikaelian I, Usary J, Hu Z et al. Identification of conserved gene expression features between murine mammary carcinoma models and human breast tumors. Genome Biol 2007; 8: R76-. 
39 Liu R, Zhou Z, Zhao D, Chen C. The induction of KLF5 transcription factor by progesterone contributes to progesterone-induced breast cancer cell proliferation and dedifferentiation. Mol Endocrinol 2011; 25: 1137-1144.

40 Kessler JD, Kahle KT, Sun T, Meerbrey KL, Schlabach MR, Schmitt EM et al. A SUMOylation-dependent transcriptional subprogram is required for Myc-driven tumorigenesis. Science 2012; 335: 348-353.

41 Peppercorn J, Perou CM, Carey LA. Molecular subtypes in breast cancer evaluation and management: divide and conquer. Cancer Invest 2008; 26: 1-10.

42 Chen ZY, Wang X, Zhou Y, Offner G, Tseng CC. Destabilization of Kruppel-like factor 4 protein in response to serum stimulation involves the ubiquitin-proteasome pathway. Cancer Res 2005; 65: 10394-10400.

43 Yoshidome K, Shibata MA, Couldrey C, Korach KS, Green JE. Estrogen promotes mammary tumor development in C3(1)/SV40 large T-antigen transgenic mice: paradoxical loss of estrogen receptor $\alpha$ expression during tumor progression. Cancer Res 2000; 60: 6901-6910.

44 Cory S, Huang DC, Adams JM. The Bcl-2 family: roles in cell survival and oncogenesis. Oncogene 2003; 22: 8590-8607.

45 Milella M, Trisciuoglio D, Bruno T, Ciuffreda L, Mottolese M, Cianciulli A et al. Trastuzumab down-regulates Bcl-2 expression and potentiates apoptosis induction by Bcl-2/Bcl-XL bispecific antisense oligonucleotides in HER-2 gene--amplified breast cancer cells. Clin Cancer Res 2004; 10: 7747-7756.

46 Dawson SJ, Makretsov N, Blows FM, Driver KE, Provenzano E, Le QJ et al. BCL2 in breast cancer: a favourable prognostic marker across molecular subtypes and independent of adjuvant therapy received. Br J Cancer 2010; 103: 668-675.

47 Huw LY, O'Brien C, Pandita A, Mohan S, Spoerke JM, Lu S et al. Acquired PIK3CA amplification causes resistance to selective phosphoinositide 3-kinase inhibitors in breast cancer. Oncogenesis 2013; 2: e83-.

48 Cerami E, Gao J, Dogrusoz U, Gross BE, Sumer SO, Aksoy BA et al. The cBio cancer genomics portal: an open platform for exploring multidimensional cancer genomics data. Cancer Discov 2012; 2: 401-404. 
49 Gao J, Aksoy BA, Dogrusoz U, Dresdner G, Gross B, Sumer SO et al. Integrative analysis of complex cancer genomics and clinical profiles using the cBioPortal. Sci Signal 2013; 6: 11.

50 Burstein HJ, Sun Y, Dirix LY, Jiang Z, Paridaens R, Tan AR et al. Neratinib, an irreversible ErbB receptor tyrosine kinase inhibitor, in patients with advanced ErbB2-positive breast cancer. J Clin Oncol 2010; 28: 1301-1307.

51 Valabrega G, Capellero S, Cavalloni G, Zaccarello G, Petrelli A, Migliardi G et al. HER2-positive breast cancer cells resistant to trastuzumab and lapatinib lose reliance upon HER2 and are sensitive to the multitargeted kinase inhibitor sorafenib. Breast Cancer Res Treat 2011; 130: 29-40.

52 Holzer RG, MacDougall C, Cortright G, Atwood K, Green JE, Jorcyk CL. Development and characterization of a progressive series of mammary adenocarcinoma cell lines derived from the C3(1)/SV40 Large T-antigen transgenic mouse model. Breast Cancer Res Treat 2003; 77: 65-76.

53 Beckers J, Herrmann F, Rieger S, Drobyshev AL, Horsch M, Hrabé de Angelis $\mathrm{M}$ et al. Identification and validation of novel ERBB2 (HER2, NEU) targets including genes involved in angiogenesis. Int J Cancer 2005; 114: 590-597.

54 Li Z, Zhao J, Li Q, Yang W, Song Q, Li W et al. KLF4 promotes hydrogen-peroxideinduced apoptosis of chronic myeloid leukemia cells involving the bcl-2/bax pathway. Cell Stress Chaperones 2010; 15: 905-912.

55 Mohan N, Ai W, Chakrabarti M, Banik NL, Ray SK. KLF4 overexpression and apigenin treatment down regulated anti-apoptotic Bcl-2 proteins and matrix metalloproteinases to control growth of human malignant neuroblastoma SK-NDZ and IMR-32 cells. Mol Oncol 2013; 7: 464-474.

56 Chen X, Xu H, Yuan P, Fang F, Huss M, Vega VB et al. Integration of external signaling pathways with the core transcriptional network in embryonic stem cells. Cell 2008; 133: 1106-1117.

57 Tiwari N, Meyer-Schaller N, Arnold P, Antoniadis H, Pachkov M, van Nimwegen E et al. Klf4 is a transcriptional regulator of genes critical for EMT, including Jnk1 (Mapk8). PLoS One 2013; 8: e57329-. 
58 Ilknur GB, Hilmi A, Tulay C, Oguz C, Selma S, Serdar S et al. The importance of extracapsular extension of axillary lymph node metastases in breast cancer. Tumori 2004; 90: 107-111.

59 Gruber G, Greiner RH, Hushchuk R, Aebersold DM, Altermatt HJ, Berclaz G et al. Hypoxia-inducible factor 1 alpha in high-risk breast cancer: an independent prognostic parameter? Breast Cancer Res 2004; 6: R191-R198.

60 Wang SL, Li YX, Song YW, Wang WH, Jin J, Liu YP et al. Triple-negative or HER2positive status predicts higher rates of locoregional recurrence in node-positive breast cancer patients after mastectomy. Int J Radiat Oncol Biol Phys 2011; 80: 1095-1101.

61 Kurebayashi J, Otsuki T, Tang CK, Kurosumi M, Yamamoto S, Tanaka K et al. Isolation and characterization of a new human breast cancer cell line, KPL-4, expressing the Erb B family receptors and interleukin-6. Br J Cancer 1999; 79: 707-717.

62 Elenbaas B, Spirio L, Koerner F, Fleming MD, Zimonjic DB, Donaher JL et al. Human breast cancer cells generated by oncogenic transformation of primary mammary epithelial cells. Genes Dev 2001; 15: 50-65.

63 Morel C, Carlson SM, White FM, Davis RJ. Mcl-1 integrates the opposing actions of signaling pathways that mediate survival and apoptosis. Mol Cell Biol 2009; 29: 3845-3852.

64 Chomczynski P, Sacchi N. Single-step method of RNA isolation by acid guanidinium thiocyanate-phenol-chloroform extraction. Anal Biochem 1987; 162: 156-159.

65 Blanquicett C, Johnson MR, Heslin M, Diasio RB. Housekeeping gene variability in normal and carcinomatous colorectal and liver tissues: applications in pharmacogenomic gene expression studies. Anal Biochem 2002; 303: 209-214.

66 Haibe-Kains B, Schroeder M, Bontempi G, Soririou C, Quackenbush J. Genefu: Relevant functions for gene expression analysis, especially in breast cancer. 2012.

67 Creighton CJ. The molecular profile of luminal B breast cancer. Biologics 2012; 6: 289297. 
68 Howe EN, Cochrane DR, Cittelly DM, Richer JK. miR-200c targets a NF-kappaB upregulated TrkB/NTF3 autocrine signaling loop to enhance anoikis sensitivity in triple negative breast cancer. PLoS One 2012; 7: e49987-. 


\section{Figure Legends}

Fig. 1. KIf4 and KIf5 are differentially expressed and positively correlated in genetically engineered mouse models (GEMMs) of breast cancer A) Microarray analysis of Klf4/5 levels across GEMMs of breast cancer. Data for 58 mammary tumors from the Gene Expression Omnibus (GSE3165) were organized by GEMM and molecular subtype. Expression values were normalized to whole mouse RNA (bars, SD). Klf4 levels in luminal and non-luminal tumors were compared via one-way ANOVA using Dunnet’s post test $(\mathrm{p}<0.0001)$. B) Spearman’s correlation was performed for the samples in panel A. C) qRT-PCR analys is of total RNA isolated from tumors of MMTV-Neu or C3(1) TAg transgenic mice (left panel). Normal mammary tissue from FVB/N mice was analyzed similarly (NL breast, $\mathrm{N}=3$ ). Tumor mean expression is depicted relative to the mean for normal tissue (bars, SE). The overall mean tumor expression of Klf4 and Klf5 was compared between GEMMs using a two-tailed t-test (for each gene, $\mathrm{p}<0.0001$ ). The $\log _{2}$ transformed data was assessed by Spearman's correlation (right panel). D) Western blot analysis of KLF4/5 levels in whole cell lysate of 10 different breast cancer cell lines. KLF expression was determined using ImageJ and normalized to $\beta$-Actin. The expression values were assessed by Spearman’s correlation (right panel). 
Fig. 2. Prognostic significance of KLF4 and KLF5 in human breast cancer. Kaplan-Meier analysis utilized a previously described breast cancer microarray database. ${ }^{40}$ Red (hi) and blue (lo) groups were defined using the median gene expression level within the tumors of breast cancer patients. A total of 364 luminal A, 175 luminal B, 239 HER2-enriched, and 287 basallike/claudin-low tumors were analyzed. A) KLF4/5 were analyzed as single variables for all tumors combined. B) KLF4/5 were analyzed as single variables within the basal-like/claudin-low and the HER2-enriched groups, as defined by PAM50 subtyping. C) The outcome of patients harboring tumors with higher expression levels of both KLF4 and KLF5 (red, hi-hi) was compared to the outcome when tumors had lower expression levels of each factor (blue, lo-lo). For all curves, significance was determined using the Log-rank (Mantel-Cox) test and $\mathrm{p}<0.05$ was considered significant. 
Fig. 3. Endogenous KLF4 and KLF5 mediate lapatinib resistance in breast cancer models. A) Levels of KLF4/5 in primary human breast tumors were determined by RNAseq (Illumina HiSeq RNAseqV2). Upper quartile normalized data were downloaded from TCGA and assigned a PAM50 subtype. Spearman's correlation was performed on the $\log _{2}$ transformed data. B) BT474 cells were treated with DMSO or lapatinib for the indicated interval. Whole cell lysate was analyzed by western blot. Expression levels from three independent experiments were determined using ImageJ for quantitation, with normalization to $\beta$-Actin (bars, SD). C) BT474 cells were treated with trastuzumab or sterile water for the indicated interval and whole cell lysate was analyzed by western blot. D) KLF4/5 transcript levels were determined by qRT-PCR following lapatinib exposure. Expression data was normalized using the housekeeping gene B2M. E) The pMIR-Report-Luc-KLF4-FL translation reporter contains as an insert within the FLuc 3' UTR the full-length KLF4 transcript, including the KLF4 protein coding region and the flanking UTRs, as previously described. ${ }^{18}$ Translation efficiency was measured by determining normalized Fluc activity in BT474 cells treated for 24 hours with lapatinib or DMSO (left panel). miR-206 levels were determined by qRT-PCR following 24 hour lapatinib exposure. Expression data was normalized using U6 snRNA (right panel). F) Cells were treated with the indicated shRNA construct, and the resulting cell populations were treated with lapatinib for 96 hours. For each cell population, cell viability relative to the DMSO control was obtained via ATP-based luminescence assay (bars, SD). G) Similarly, the lapatinib effect on the relative cell viability of M6 cells expressing ectopic KLF4 and/or KLF5 was determined. Empty vector served as a control. H) To assess mitochondrial membrane integrity, M6 cells were treated with lapatinib for 24 hours, stained with $250 \mathrm{nM}$ of Mitotracker dye, and analyzed by flow cytometry. I) To assess activity of the intrinsic apoptotic pathway, caspase-9 levels were determined in M6 cells 
expressing shCtl, shKLF4, shKLF5, or shKLF4/5. Cells were treated with lapatinib for 24 hours prior to preparation of cell extracts. *, $\mathrm{p}<0.05$; **, $\mathrm{p}<0.01$; ***, $\mathrm{p}<0.001$. 
Fig. 4. KLF4 and KLF5 cooperate to promote malignant properties in M6 cells, a HER-2 overexpressing mammary cancer model. A) Anchorage independence was assessed by incubation of the indicated cell populations in soft agar for 14-days ( $\mathrm{N}=3$, bars, $\mathrm{SE})$. B) Anchorage independence of HMLE cells expressing ectopic KLF4 and KLF5 was determined as previously described. Empty vector (-) served as a control so that all cell populations were treated with equal volumes of lentiviral supernatant. C) Cells were injected into the mammary gland of female athymic mice and tumor xenograft volume was monitored over a period of several weeks (left panel, $\mathrm{N}=5$; bars, SE). Similar effects on tumor burden were obtained using distinct shRNAs for the suppression of each Kruppel-like factor (right panel, N=5; bars, SE). D) Cell death was determined by trypan blue exclusion following 24 hours of matrix deprivation for the indicated cell populations ( $\mathrm{N}=3$; bars, $\mathrm{SE}$ ). E) As an independent method, cell death due to matrix deprivation in M6 cells was determined by fluorescence microscopic imaging of 4',6diamidino-2-phenylindole (DAPI)/ propidium iodide (PI) stained cells. Results were quantitated using ImageJ (two-tailed t-test; $\mathrm{N}=3$; bars, SE). *, $\mathrm{p}<0.05 ; * *, \mathrm{p}<0.01 ; * * *, \mathrm{p}<0.001$ 
Fig. 5. KLF4 and KLF5 depletion is associated with reduced expression of anti-apoptotic BCL2 family members. A) BT474 cells were treated with DMSO, $1 \mu \mathrm{M}$ lapatinib, sterile water or $10 \mu \mathrm{g} / \mathrm{mL}$ trastuzumab for the indicated time intervals. BCL2, BCL-XL and MCL1 levels were determined by western blot analysis. B) Protein expression was analyzed in control or KLF-depleted BT474 and KPL4 cells. C) Protein expression (left panel) and mRNA expression (right panel) was analyzed in the indicated cell populations. D) Protein expression was analyzed in control HMLE cells and in cells expressing ectopic KLF4 and/or KLF5. E) Spearman's correlation between KLF4, KLF5 and MCL1 levels as determined by RNAseq analysis of 890 human breast tumors. F) The impact of KLF4/5 knockdown on the lapatinib-mediated induction of MCL1 was determined in BT474 cells. Cells were treated with $250 \mathrm{nM}$ lapatinib or DMSO for 24 hours. For three independent experiments the expression levels were quantitated using ImageJ and normalized to $\beta$-Actin (bars, SD). G) MCL1 levels were reduced by siRNA and the resulting cell populations were treated with lapatinib for 96 hours. For each cell population, cell viability relative to the DMSO control was obtained via ATP-based luminescent assay (paired t-test, two tailed; bars, SD). H) Similarly, lapatinib resistance was analyzed in KLF4/5 knockdown BT474 cells following rescue with exogenous MCL1 expression vector or empty vector control. $\mathrm{p}<0.05$; $* *, \mathrm{p}<0.01 ; * * *, \mathrm{p}<0.001$ 
Table 1. The expression levels of KLF4/5 in breast cancer are mutually inclusive with the anti-apoptotic BCL2 family members

\begin{tabular}{|l|l|l|l|l|r|}
\hline Gene & BCL-XL & BCL2 & MCL1 & KLF5 & \multicolumn{1}{|c|}{ KLF4 } \\
\hline BCL-XL & --- & $1.22 \mathrm{E}-1$ & $1.59 \mathrm{E}-1$ & $1.78 \mathrm{E}-1$ & $\mathbf{1 . 0 0 E - 2}^{\mathbf{1}}$ \\
\hline BCL2 & & --- & $\mathbf{6 . 9 8 E - 4}$ & $\mathbf{2 . 3 0 E - 5}^{\mathbf{1}}$ & $2.52 \mathrm{E}-1$ \\
\hline MCL1 & & & --- & $\mathbf{1 . 2 8 E - 2}^{\mathbf{1}}$ & $\mathbf{9 . 0 0 E - 6}^{\mathbf{1}}$ \\
\hline KLF5 & & & & --- & $<\mathbf{1 . 0 0 E - 6}^{\mathbf{1}}$ \\
\hline KLF4 & & & & & --- \\
\hline
\end{tabular}

Using cBioPortal, the mutual inclusivity/exclusivity of KLF4/5 and anti-apoptotic BCL2 family member expression was assessed in 958 human breast tumors using a +/- 1.5 z-score range.

Transcript abundance was determined by microarray. P-values were obtained by Fisher's exact T-test (bold indicates significance). ${ }^{1}$ Denotes an odds ratio (OR) range of 2-10. For this analysis, OR $>2.0$ signifies co-occurrence of expression within the tumor subsets as defined by the zscore. 


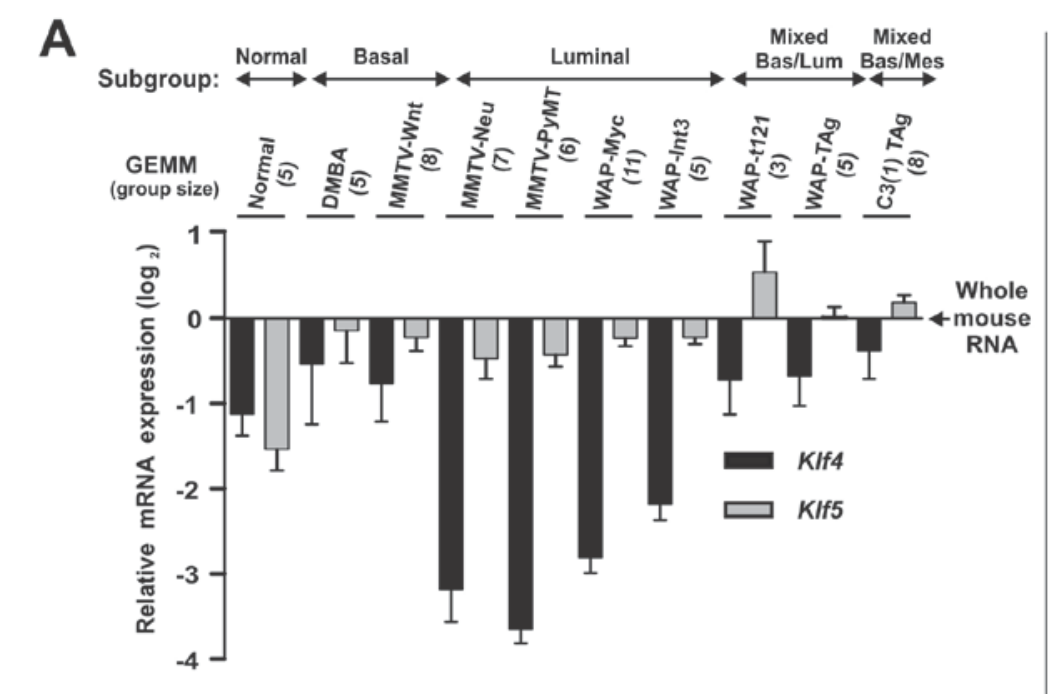

B Klf4 vs KIf5 (All GEMMs)

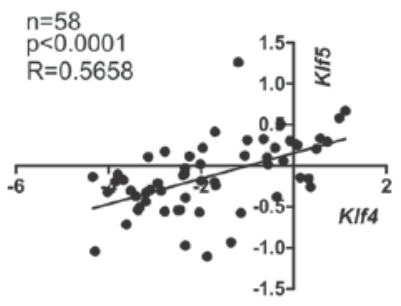

KIf4 vs KIf5 (Basal GEMMs)

$n=13$
$p=0.0005$

$\mathrm{p}=0.0005$
$\mathrm{R}=0.8242$

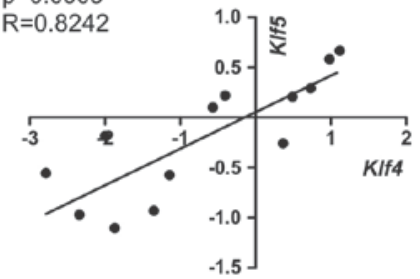

\section{C}
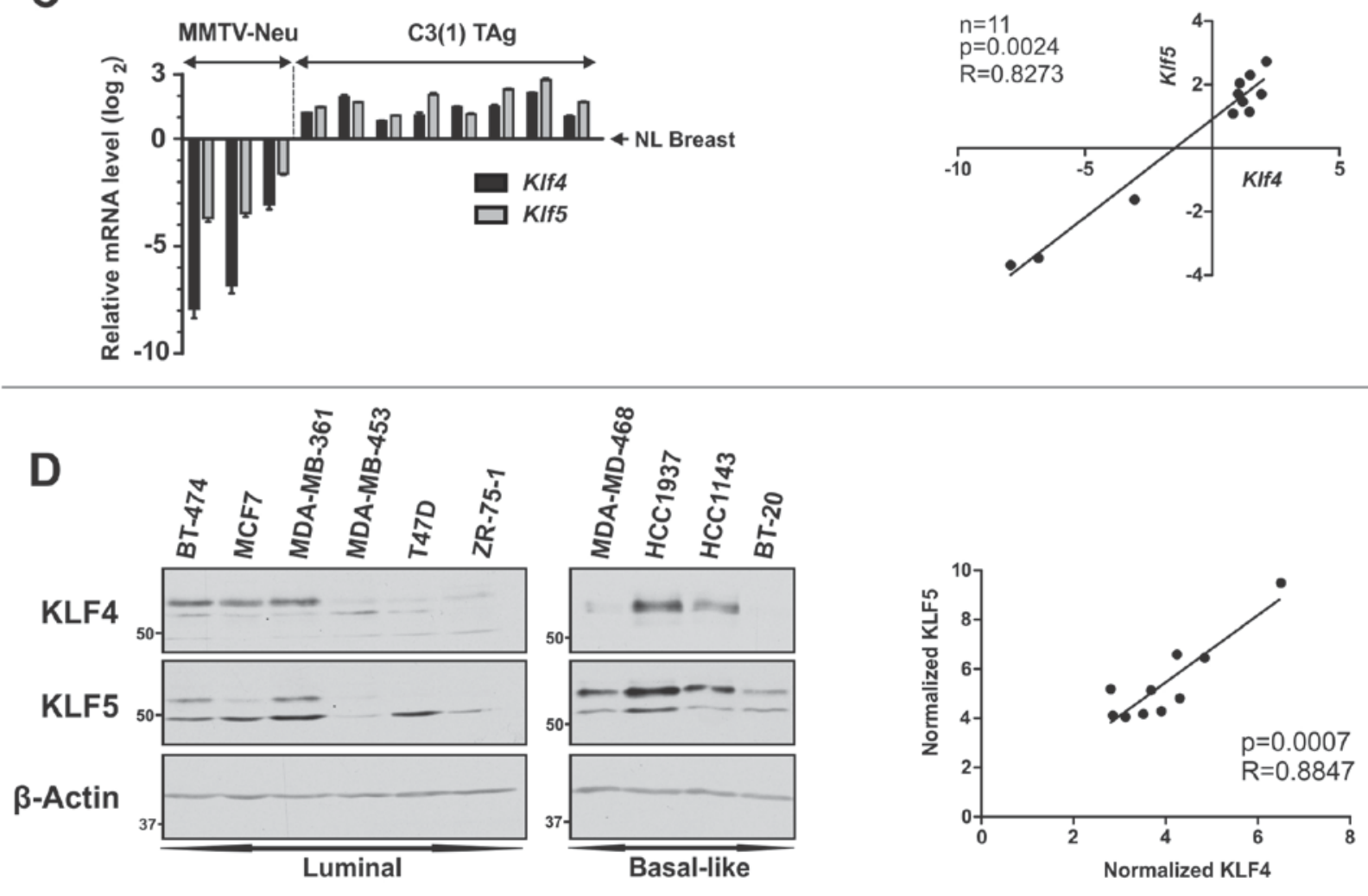

Fig. 1 
A

$K L F 4$ in all tumors
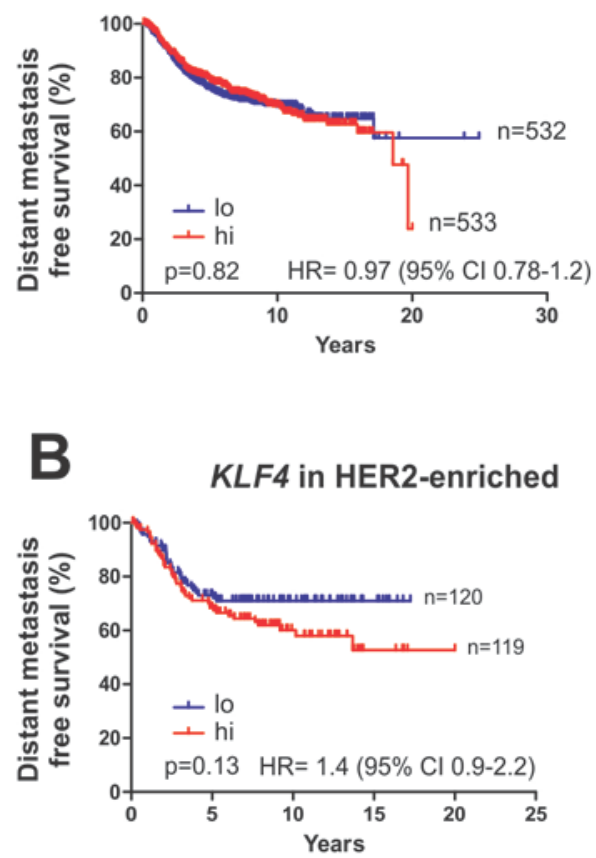

KLF4 in Basal-like/claudin-low

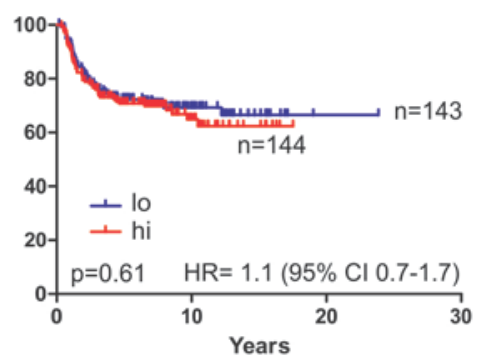

C

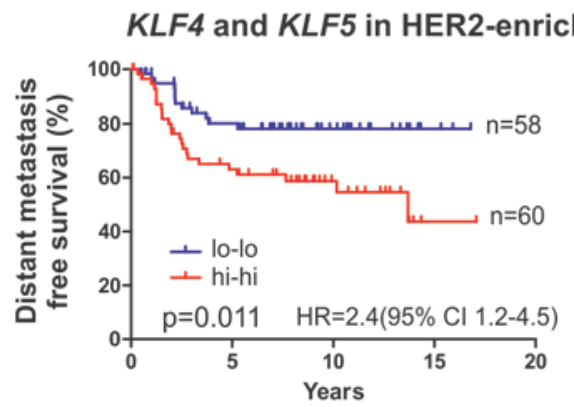

$K L F 5$ in all tumors

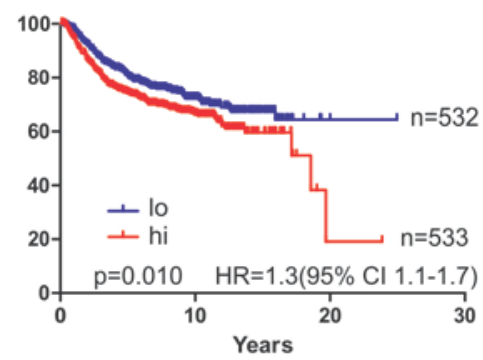

KLF5 in HER2-enriched

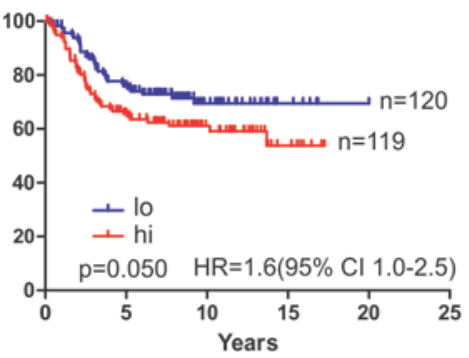

$K L F 5$ in Basal-like/claudin-low

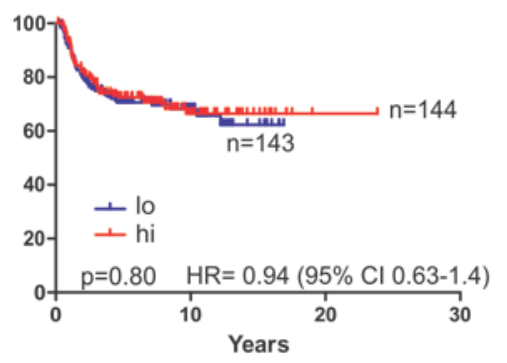

KLF4 and KLF5 in Basal-like/claudin-low

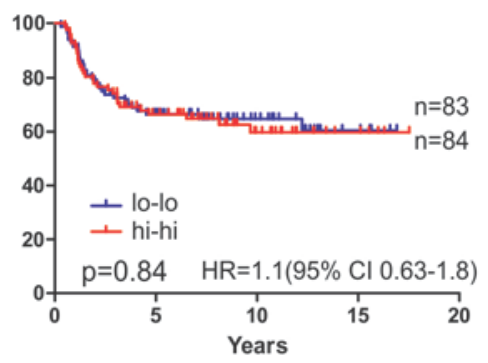

Fig. 2 
A

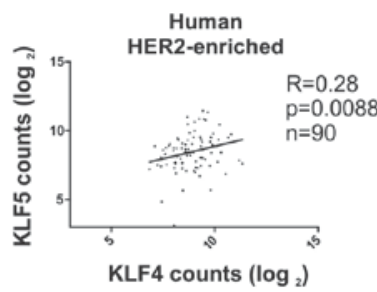

B

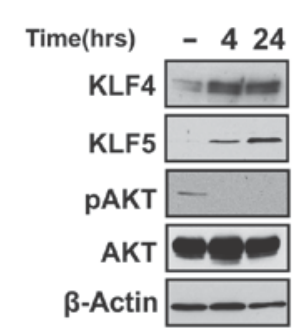

BT474 - Lapatinib $(1 \mu \mathrm{M})$

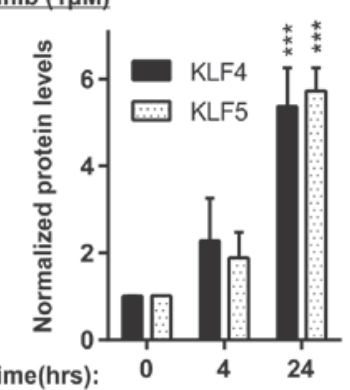

E

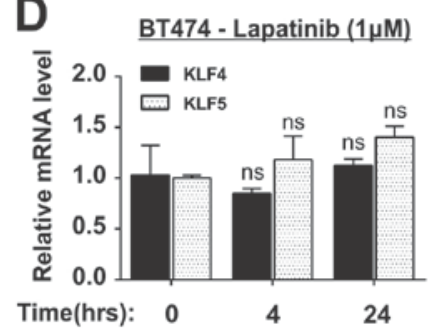

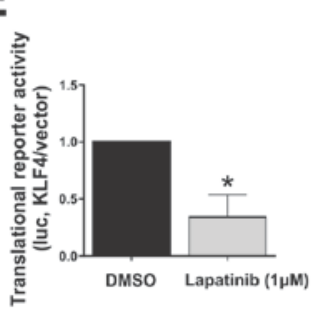

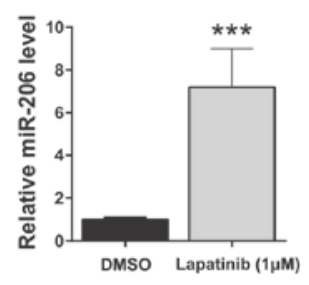

F
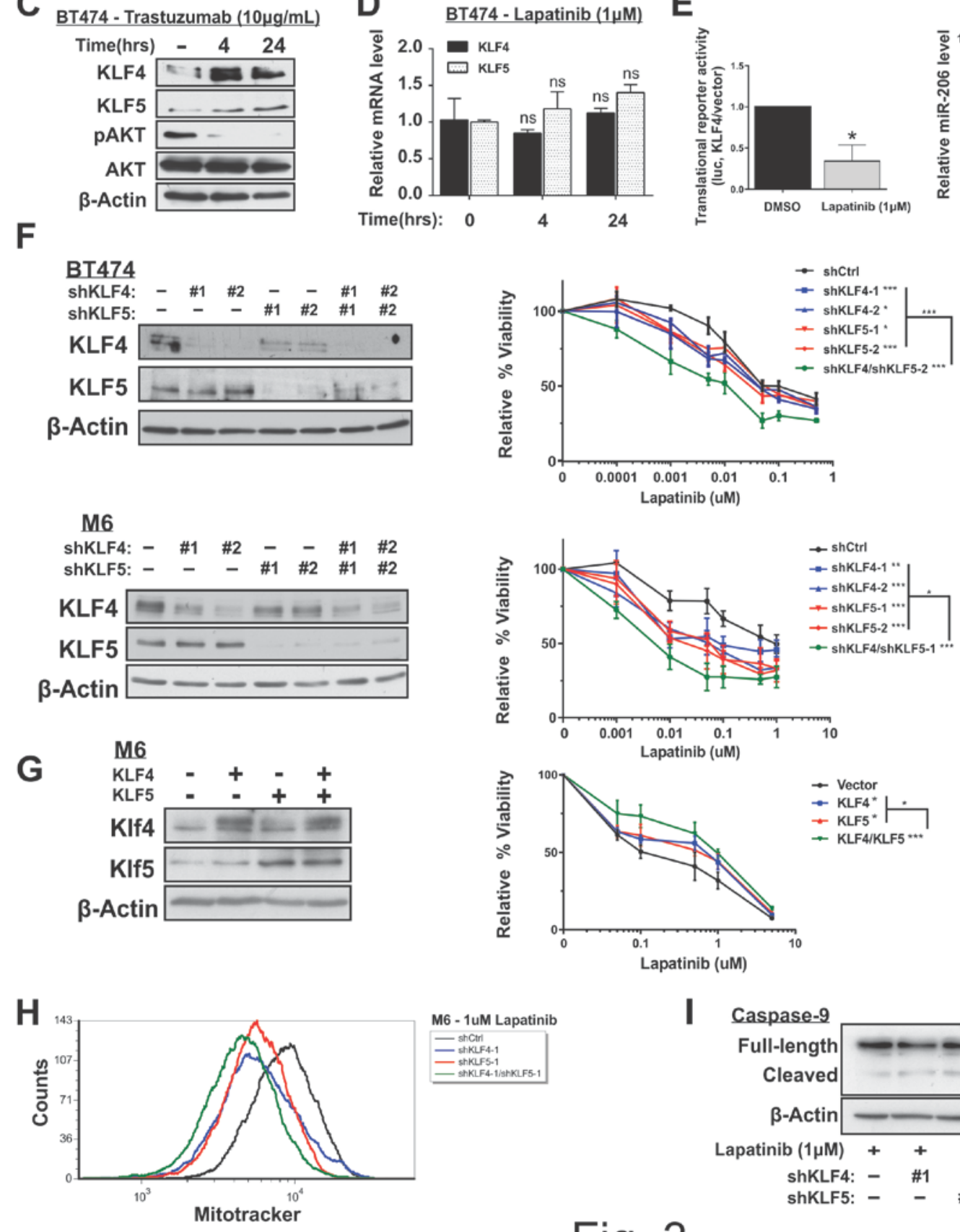

Fig. 3

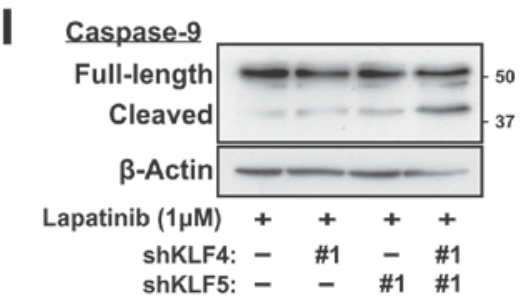


A
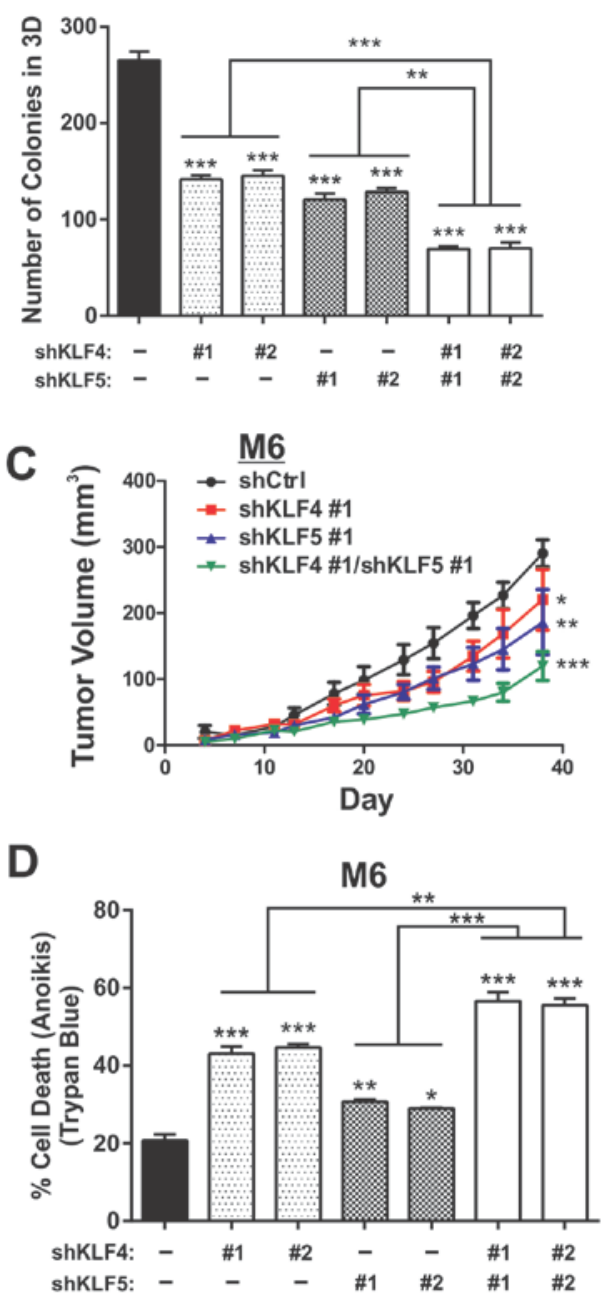

B

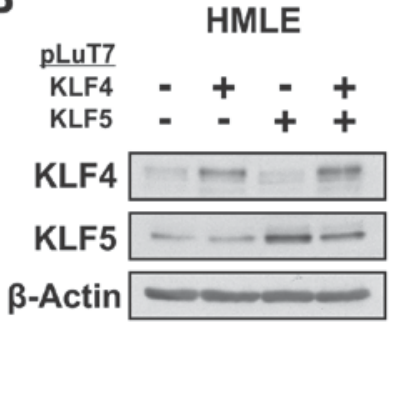

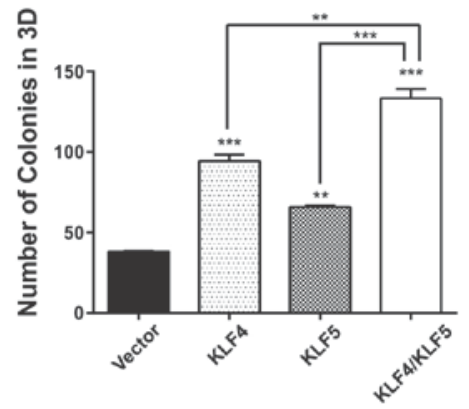

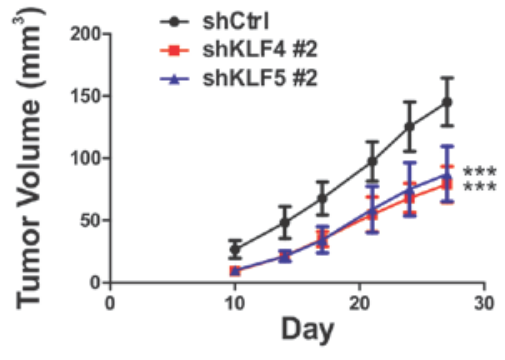

HMLE

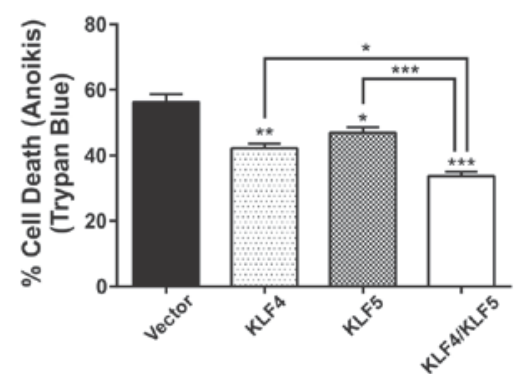

Merge
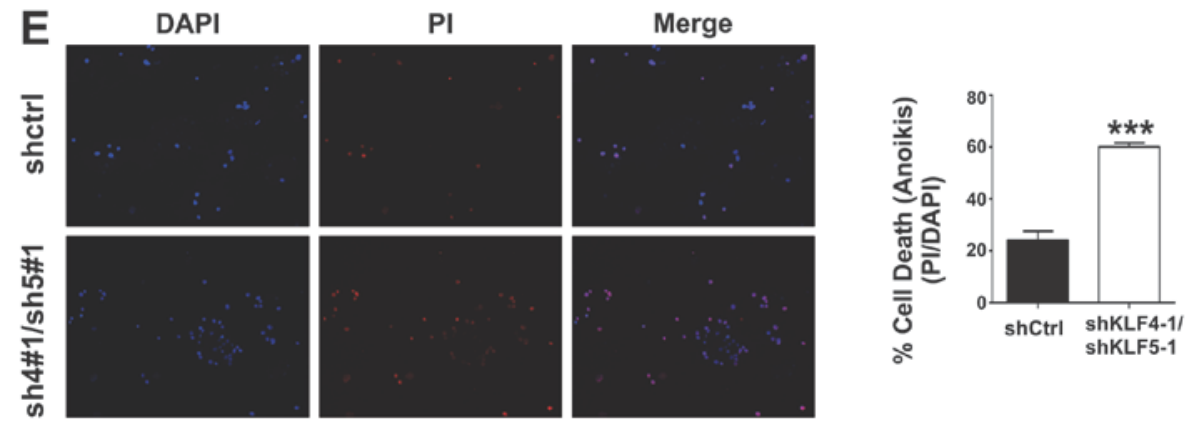

Fig. 4 
A Lapatinib $(1 \mu \mathrm{M})$ Trastuzumab $(10 \mu \mathrm{g} / \mathrm{mL})$ Time (hrs) - 424 Time (hrs) - 424 BCL2 BCL-XL $\cdots$ MCL1 $\ldots$ $\beta$-Actin BCL2 BCL-XL … MCL1 ‥ $\beta$-Actin
B

BT474

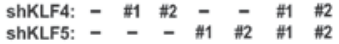
BCL2 $\square=m-m$

BCL-XL

MCL1 $\longrightarrow \cdots$

$\beta$-Actin $-\infty$

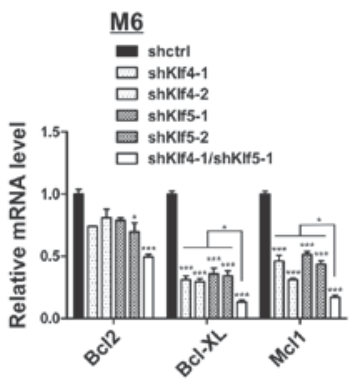

KPL4

ShKLF4: - \#1 \#2 $\quad-\quad-\quad \# 1 \quad \# 2$

KLF4 $\infty-\infty 60$

KLF5 $\ldots$

BCL2 n.d.

$\mathrm{BCL}-\mathrm{X}+-$

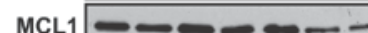

B-Actin

D

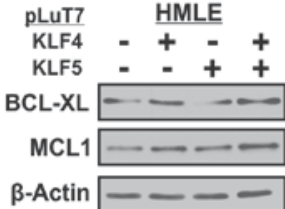

$\beta$-Actin $--\infty-\infty$
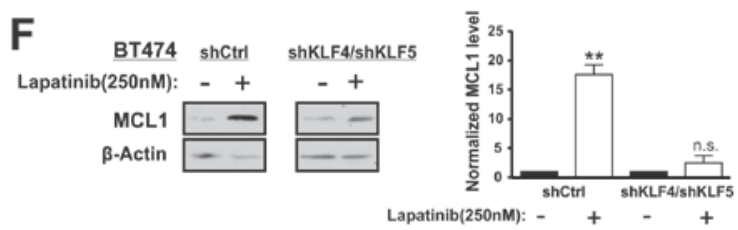

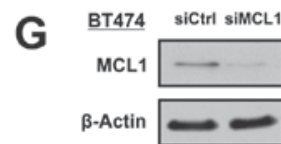

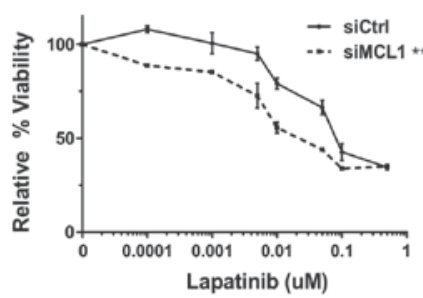

H BT474-shKLF4/shKLF5 Vector: ctrl MCL1
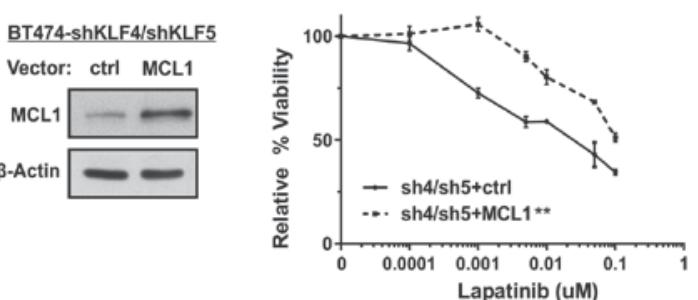

Fig. 5 


\section{A}

Luminal A
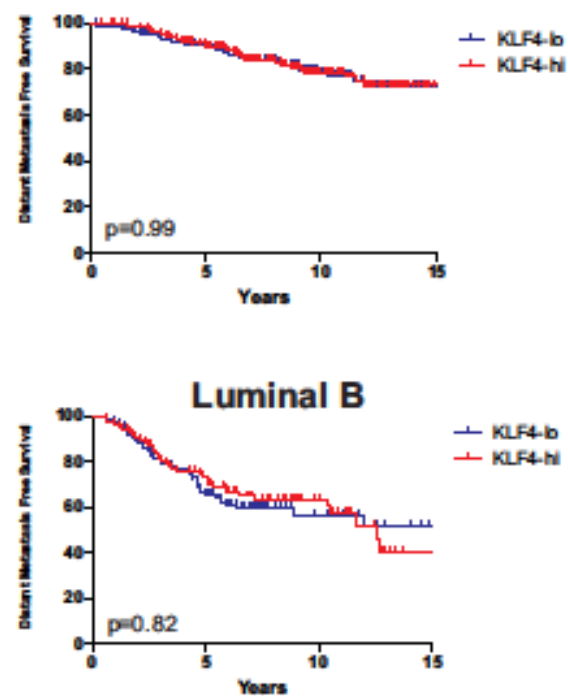

Luminal A
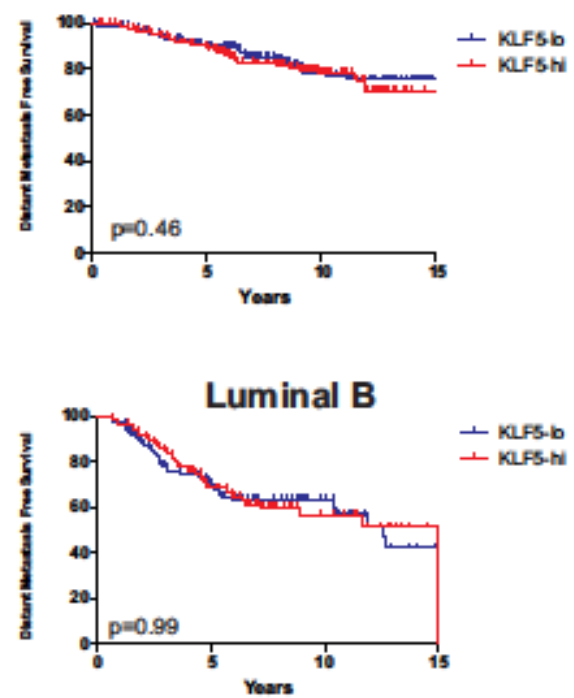

B

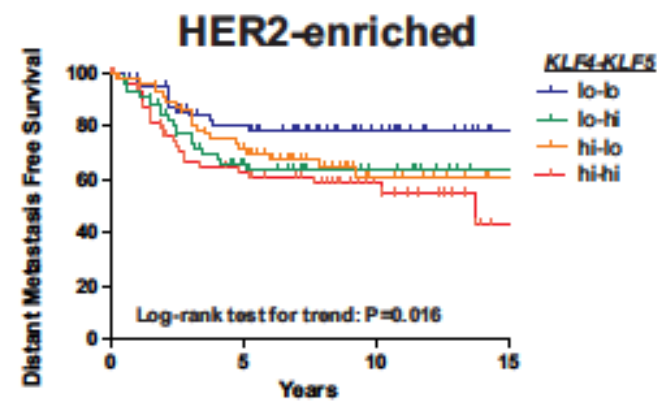

Supplemental Fig 1. DMFS analysis. A. Luminal subtype tumors were analyzed. B. The four subgroups of HER2-enriched breast cancers identified by $K L F 4$ and $K L F 5$ expression were analyzed. Patients were assigned using median expression of $K L F 4$ (high or low) and $K L F 5$ (high or low). 


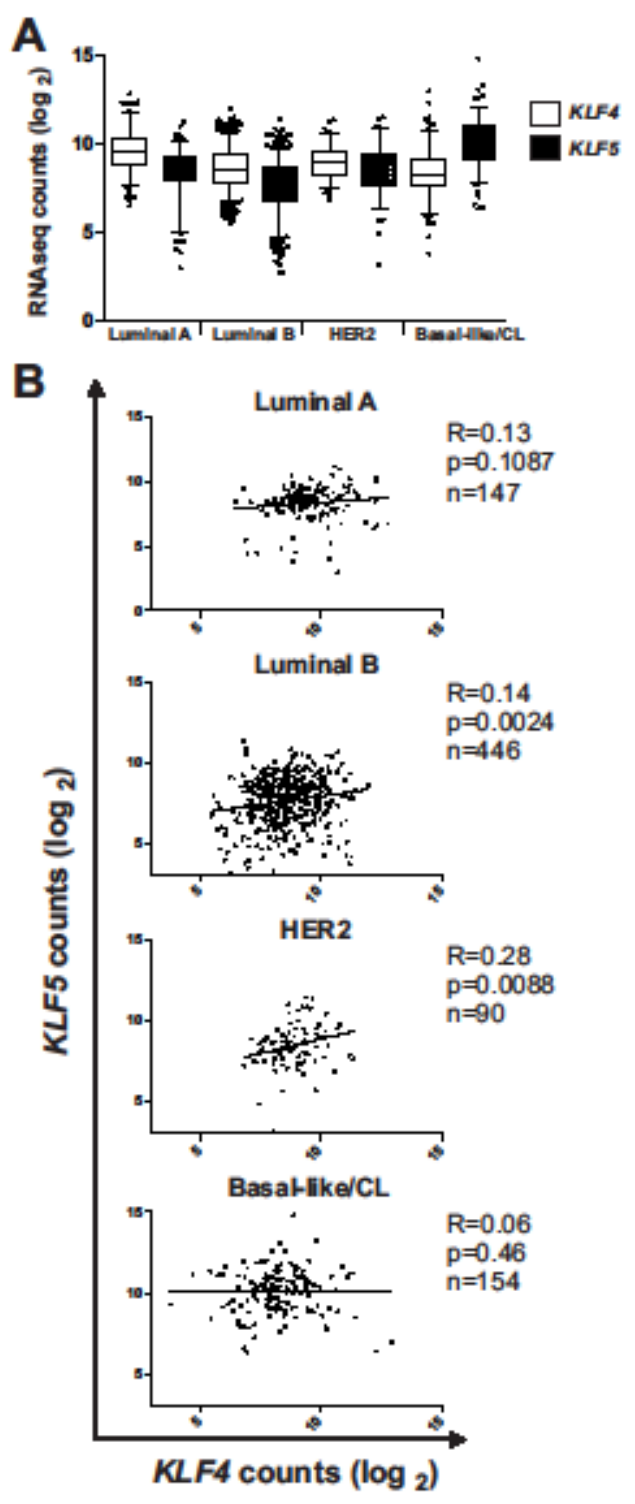

Supplemental Fig. 2. RNAseq analysis of $K L F 4$ and $K L F 5$ in primary human breast cancer samples. A) Upper quartile normalized RNAseq (Illumina HiSeq RNAseqV2) data were downloaded from TCGA and assigned a PAM50 subtype with expression displayed as a box-and-whisker plot. B) $K L F 4$ and $K L F 5$ were assessed by Spearman's correlation. 
Primer (5'-3')

Gene

\begin{tabular}{|l|l|}
\hline GGACCCGAGAAGACCTCCTT & musRPLP0-F \\
\hline GCACATCACTCAGAATTCAATGG & musRPLP0-R \\
\hline GTGGTAAGGTTCTCGCCTGT & MusKLF4_R_2 \\
\hline GGAAAAGAACAGCCACCCAC & MusKLF4_F_2 \\
\hline GGTCTACCACTGAGGCACTG & MusKLF5_R_2 \\
\hline GAGCTGGTCCAGACAAGATGT & MusKLF5_F_2 \\
\hline ATGCCTTTGGGAACTATATGGC & musBCL2-F \\
\hline GGTATGCACCCAGAGTGATGC & musBCL2-R \\
\hline AGTGGAGGTACACCCCTCAG & musBCL-X_F \\
\hline AAGGCTGGGATCACAAACGTG & musBCL-X_-R \\
\hline GACGACCTATACCGCCAGTC & musMCL1_F \\
\hline AGAGGCTTCGAGTCCTTGGA & musMCL1-R \\
\hline TCCCAGGTACACTTGTATGGC & HsKLF5-R \\
\hline ACCCTGGTTGCACAAAAGTT & HsKLF5-F \\
\hline AGAGTTCCCATCTCAAGGCA & HsKLF4-R \\
\hline GTCAGTTCATCTGAGCGGG & HsKLF4F \\
\hline
\end{tabular}

Supplemental Table 1: qRT-PCR primers. Listed primers were used to assay transcript levels of the respective genes. Primers are listed 5'-3'. 


\section{$\underline{\text { Chapter } 3}$}

Distinct functions and effector signaling of KLF4 and KLF5 in breast cancer

Note: This chapter represents unpublished data relevant to this topic 


\begin{abstract}
In the family of Kruppel-like factors (KLF), KLFs 2, 4 and 5 (termed “core KLFs") were found to redundantly maintain pluripotency in embryonic stem (ES) cells. Although traditionally considered to be either uninvolved or antagonistic to each other in adult tissues, KLF4 and KLF5 work in concert to promote drug resistance to targeted therapies in HER2-enriched breast cancer. Therefore, it is possible that these KLFs function in tandem in other cancer-relevant properties as well. We explored several known features and targets of KLF4, determining if the other core KLFs (KLF2 and KLF5) could likewise participate. In experimental settings, KLF4 paradoxically promotes malignant properties while also slowing cell growth. Interestingly, such effects could not be confirmed in vivo as KLF4 high tumors exhibit no detectable markers of slowed proliferation. Although individual KLF4 depletion enhanced proliferation, reducing both KLF4 and KLF5 ameliorated this effect. As KLF4 and KLF5 are correlated in breast tumors, this suggests that their coexpression remedies the growth inhibitory effects of KLF4. Despite their overlapping signaling in ES cells, KLFs 2 and 5 could not restore CD44 expression in KLF4 depleted cells, arguing for distinct functions on this setting. Moreover, KLF4, not KLF2 or KLF5, significantly enhanced Notch signaling further demonstrating unique functions of this KLF. Similar to their functions in ES cells, KLF4 and KLF5 can exhibit both overlapping and distinct targets in breast cancer, imparting additional complexity in our understanding of these genes in this disease.
\end{abstract}




\section{Introduction:}

Kruppel-like factors are a family of 17 transcription factors that participate in a number of diverse physiological processes, often regarding cell fate. In certain tissues, KLFs can demonstrate unique relationships between members of the family. For example, KLFs 2, 4, and 5, termed “core KLFs", can redundantly maintain the stem cell phenotypes in ES cells. ${ }^{1}$

Additionally, KLF4 and KLF5 were recently shown to correlate and cooperatively promote malignant features in breast cancer (Chapter 2). As the relationship of these factors are involved in key biological processes, delineating the mechanistic and functional details of these interactions remains an attractive area of research.

The oncogenic activity of Klf4 in primary mouse fibroblasts is contingent upon neutralization of its cytostatic effects, for example by deletion of $C d k n 1 a\left(\mathrm{p} 21^{\text {Waf1/Cip } 1}\right){ }^{2,3}$ Relevant to the paradoxical observations noted above, cancer cells may coexpress other factors that mitigate growth suppression by KLF4. An attractive candidate to mediate this function is KLF5 which promotes cell proliferation in a variety of contexts, and regulates the expression of several cell cycle regulatory proteins in a fashion opposite to KLF4. ${ }^{4}$ In epithelial tissues such as the mucosal lining of the gut, these two factors are expressed in distinct compartments, with KLF5 localized to proliferative cells within the crypts and KLF4 to differentiated, post-mitotic cells. $^{4,5}$ Analysis of KLF haploinsufficient mice identified opposing effects of Klf4 and Klf5 on colorectal tumorigenesis, consistent with a tumor suppressor role of Klf4 and with protumorigenic signaling by Klf5. ${ }^{6,7}$ In a different context, Klf4 and Klf5 appear to function in concert to promote anterior eye development, including goblet cell formation in the surface epithelium. ${ }^{8,9}$ 
Recently, KLF4 and KLF5 were found to promote lapatinib resistance in HER2overexpressing breast cancer cells through the maintenance of anti-apoptotic signaling (Chapter 2). Although in this instance these factors were studied in tandem, there are still several other attractive biological and molecular processes in breast cancer where this relationship can be explored. KLF4 promotes oncogenic non-canonical Notch1 signaling in the transformation of immortalized rat kidney epithelial cells and enhances cancer stem cell characteristics in breast cancer. $^{10,11}$ Although KLF4 and KLF5 are correlated and breast cancer, it is unclear whether they share common or distinct molecular outputs. Consequently, we describe shared, antagonistic and distinct functions of KLF4 and KLF5 in breast cancer.

\section{Materials and Methods}

\section{Cell culture and plasmid transduction}

HMLE and MDA-MB-231 cells were cultured as previously described. ${ }^{12}$ cDNA for KLF2 and KLF5 was purchased from Origene and Open Biosystems respectively. KLF2 was HA-tagged via PCR cloning and primer sequences are listed in Supplemental table 1. HA-KLF2 was cloned in retroviral vector pBABE-Neo. HA-KLF4 was cloned into in the pLJD retroviral vector previously described and KLF5 was cloned in the lentiviral vector, pLuT7. The shKLF4-1 and control vector were previously described and shKLF4-2 vector (V2LHS_28349) Viral particles

were constructed as previously described. ${ }^{12}$ KLF4 and KLF5 were overexpressed in HMLE cells as previously described (Chapter 2)

Immunoblot analysis 
Primary antibodies were KLF2 (Aviva systems biology), KLF4 (H180, Santa Cruz), KLF5 (Millipore), and $\beta$-Actin (C-4, Sigma). Gel electrophoresis, transfer, and immunoblot analysis was performed as described. ${ }^{12}$

Quantitative, real-time, reverse transcription and PCR (qRT-PCR) analysis of mRNA

Total RNA from cell lines was extracted using the RNeasy minikit (Qiagen). qRT-PCR was performed as previously described. ${ }^{12}$ Primers used for qRT-PCR can be found in Supplemental table 1.

Anoikis assays

Cell death as a result from suspension cultured was assessed as previously noted (Chapter 2).

Flow cytometry

$5.0 \times 10^{5}$ MDA-MB-231 cells were washed with PBS and suspended in 1\% BSA(MP Biochemicals) in PBS. To block, $1 \mu \mathrm{g}$ human IgG (R\&D; 1-001-A) was added to a final concentration of $0.5 \mu \mathrm{g} / \mu \mathrm{L}$ for 20 minutes on ice. Anti-CD44-APC (cat. \#560890 ,BD Pharmigen) and Anti-CD24-PE(cat.\# 555428,BD Pharmigen) were added to the cells as the manufacturers instructed and were allowed to incubate on ice for 20 minutes. Cells were washed with $1 \% B S A / P B S$ and resuspended to $1.0 \times 10^{6} \mathrm{cells} / \mathrm{mL}$. Stained cells were analyzed in a BD FACS Calibur flow cytometer using BD CellQuest Pro software. Plots were generated using FCS Express 4 (De novo software).

\section{Luciferase reporter assays}


Reporter assays were performed as previously described using the previously characterized Hes1 luciferase reporter construct. ${ }^{10}$

\section{Chromatin-Immunoprecipitation assay}

ChIP protocol was adapted from Boyd and Farham. HMLE cells expressing KLF4 were crosslinked for 10 minutes at room temperature in 1\% formaldehyde (Fisher \# PI28908) and crosslinking was stopped by addition of glycine to a final concentration of $0.125 \mathrm{M}$. Cell membranes were disrupted, nuclei were lysed, and collected chromatin complexes were sheared using Disruptor $^{\mathrm{TM}}$ (Diagenode) sonicator. Sheared chromatin was immunoprecipitated with $5 \mu \mathrm{g}$ of either anti-KLF4 (Santa Cruz, H-180) or normal rabbit IgG (Sigma) and protein A-sepharose beads (Sigma, P3391). Cross-linking was reversed and immunoprecipitated DNA was purified using QIAquick PCR purification kit (Qiagen). Primers were designed to amplify sequence complementary to two regions on the Hes5 promoter, regions A or B. Refer to supplemental table 1 for primer sequences.

Gene set enrichment analysis

ChIP-seq databases published by Chen et al. and Li and colleagues for direct targets of KLF4 and N1ICD/RBPJ respectively, were cross referenced to ascertain common genes. ${ }^{13,14}$ Of the observed targets for KLF4 and N1ICD/RBPJ, only genes with a gene association score $>0.9$ for KLF4 or a P-value <1.0x10-4 N1ICD/RBPJ for were included. Common targets were identified using the statistical software program $\mathrm{R}$ (version 3.0.1). Using this list of genes, KEGG pathway analysis was performed via WebGestalt (http://bioinfo.vanderbilt.edu/webgestalt/).

Statistical analysis 
Statistical analyses were performed in GraphPad Prism 6 (GraphPad Software). RNA samples were independently analyzed by qRT-PCR three times in duplicate fashion (column, mean; bars, SE). Unless otherwise indicated, all experiments were performed three times in triplicate.

Differences were considered significant when two-tailed p-values were $<0.05$.

\section{Results}

Complementary and cooperative effects of endogenous Klf4 and Klf5

As compared to other GEMMs, Klf4 and Klf5 are enriched in C3(1) TAg tumors.

Therefore M6 cells represent an attractive context for functional studies. We assessed the role of endogenous Klf4 and Klf5 using shRNA-mediated knockdown (Fig. 1A). Suppression of Klf4 significantly enhanced the proliferation of M6 cells in 2D culture, while depletion of Klf5 only modestly slowed cell growth (Fig. 1B). In this model, simultaneous knockdown of Klf4 and Klf5 abrogated the increased proliferation of the Klf4-depleted cells, consistent with opposing roles for KLF4 and KLF5, as proposed. ${ }^{4,5}$ Similar results were obtained using an independent shRNA to target each factor (data not shown).

We evaluated several known KLF target genes known to participate in the cell cycle, and found Ccnb1 to be differentially regulated by Klf4 and Klf5 (Fig. 1C). ${ }^{15,16}$ Knockdown of Klf4 increased Ccnb1 expression and reduced the proportion of cells in the G2/M phases, whereas Klf5-depletion decreased Ccnb1 mRNA (Fig. 1C and data not shown). Consistent with their complementary effects on cell proliferation, suppression of Klf5 in the Klf4-depleted cells restored Ccnb1 to levels similar to control.

Non-redundant functions of the core KLFs in breast cancer

KLF2, KLF4 and KLF5 ("core KLFs") are redundant for the maintenance of the stem cell phenotype in mouse embryonic stem cells. ${ }^{1}$ Given that KLF4 and KLF5 are also correlated and 
coregulated in breast cancer, they may contribute to overlapping oncogenic processes (Chapter 2). Therefore, we investigated this hypothesis by rescuing KLF4 knockdown by forced expression of each core KLF (Fig. 2A, B). Functionally, knockdown of KLF4 enhances MDAMB-231 cell's sensitivity to anoikis-mediated cell death (3-fold) (Fig. 1A) induced by 24-hours in suspension culture. However, forced expression of only KLF4 and not KLF2 or KLF5 rescued anoikis resistance as measured by either trypan blue exclusion or fluorescent microscopy (Fig. 2B, C).While it has been previously reported that KLF4 depletion results in a reduction in the CD44 ${ }^{+} / \mathrm{CD} 24^{-}$population, in our hands shKLF4 cells exhibited reduced surface CD44 with no significant changes in the CD44 $/$ CD24- fraction (Fig. 2D). ${ }^{11}$. Forced expression of vector, KLF2 and KLF5 did not rescue the decrease in CD44 signal, however rescue with KLF4 resulted in a curve similar to control cells and exhibited significantly higher surface CD44 than the other rescue settings (Fig. 2D). Together, these data suggest that the core KLFs can exhibit nonredundant functions in breast cancer.

\section{KLF4 functions in canonical Notch1 signaling}

To address distinct molecular outputs of KLF4 and KLF5 in breast cancer, we examined their influence on the pathway Notch1. KLF4 impacts Notch signaling in a variety of tissues and moreover this association is implicated in tumorigenesis. ${ }^{10,11,17}$ To further investigate the interaction between KLF4 and Notch, we analyzed two ChIP-seq databases in which direct targets for KLF4 and N1ICD/RBP-Jא were catalogued in embryonic and neural stem cells respectively. ${ }^{13,14} \mathrm{RBP}-\mathrm{J} \kappa$, also known as $\mathrm{CBF}-1$, is an essential component of the activated Notch complex responsible for the transcriptional upregulation of Notch pathway target genes. Cross referencing the most highly predicted targets using the statistical program $\mathrm{R}$ revealed a significant overlap in target genes (Fig. 3A). Furthermore, KEGG pathway analysis of the 
common targets illustrated a significant association with pathways known to be dysregulated in cancer, such as Wnt and Ras (Table 1). In addition to co-targeting similar genes, KLF4 was previously shown to bind to the promoter of Notch1, a target gene of Notch signaling. Therefore, we hypothesized that KLF4 promotes Notch signaling transcriptionally. Using JASPAR CORE, another Notch target gene, Hes5, was shown to have several highly predicted binding sites for KLF4 in its promoter region, many of these sites near putative sites for N1ICD/RBP-JK (Fig. 3B). qPCR of site A (-939 to -772) showed a 3.3-fold enrichment in ChIP with anti-KLF4 versus IgG control, while site B (-442 to -247) showed no comparative difference. Additionally, forced expression of KLF4 in HMLE cells promoted mRNA levels of canonical targets Notch1, Hes5 and Hey1 (Fig. 3C) as well as increasing luciferase activity on the Hes1 promoter (Fig. 3D) while showing no effects on Notch1 processing or ligands (data not shown). KLF5 does not appear to significantly impact Notch signaling or KLF4's effect in this setting, suggesting distinct functions for the two KLFs.

\section{Discussion}

Although dispensable for embryonic development, KLF4 promotes critical developmental events including formation of the cutaneous water permeability barrier and other epithelial features such as goblet cell differentiation. ${ }^{9,18-21}$ Despite these pro-differentiation effects and its normal localization to post-mitotic epithelial cells, in model systems KLF4 induces malignant transformation. ${ }^{3,22}$ In addition, it functions as a pluripotency factor in both ES cells and somatic cells. ${ }^{1,23}$ Consistent with this dual identity, in experimental models of

mammary cancer it is clear KLF4 can either promote or suppress malignant properties. ${ }^{11,21,24,25}$ Pro-tumorigenic signals may include transcriptional suppression of p53 and the induction of Notch1. ${ }^{3,10}$ Tumor suppressor effects can be ascribed to its suppression of cell proliferation 
and/or its potential for inhibiting metastasis, possibly by regulating the epithelial mesenchymal transition. ${ }^{20,21,24}$ As a major regulators of cell fate, a better understanding of downstream pathways that mediate KLF signaling can enhance our understanding of how they contribute to the cancer process.

The pluripotency of mouse ES cells is maintained by the redundant function of three “core” KLFs, including Klf2, Klf4, and Klf5. ${ }^{1}$ Suggesting an overlap in function, suppression of all three results in differentiation and the loss of stem cell markers, and each is sufficient to restore the ES phenotype when reintroduced into the differentiated cells. In contrast to KLF4, KLF5 is a well-established promoter of cell proliferation. ${ }^{4}$ Peeper and colleagues showed that KLF4-mediated transformation is contingent upon disruption of its cytostatic effect, for example by deletion of Cdkn1a or by enforced expression of RAS or Cyclin $\mathrm{D} 1{ }^{3}$ We elaborated on this concept through the relationship between KLF4 and KLF5.

In addition to coregulating anti-apoptotic signaling, we observed a complementary relationship between KLF4 and KLF5 on cellular growth. Although these factors were molecularly antagonistic, as demonstated by their opposing effects on Ccnb1 levels, the overall result of their coexpression was phenotypically beneficial with regards to proliferation. In this setting, KLF5 acted similarly to Cdkn1a deletion/Cyclin D1 overexpression through its neutralizing effect on KLF4-mediated growth inhibitory signaling. These observations, in addition to their function in drug resistance, argue that KLF4 and KLF5 can act as a protumorigenic pair in breast cancer.

Although the KLFs can be redundant in ES cells, it appears this relationship is not preserved in breast cancer. CD44 expression is often associated with cancer stem cell characteristics and depletion of KLF4 reduced surface CD44 as determined by flow cytometry. ${ }^{26}$ 
Only KLF4, not KLF2 or KLF4, could rescue this particular deficit, arguing to distinct functions in this setting.

In agreement, KLF4 and KLF5 do not necessarily exhibit shared interactions in all their respective effector pathways. In RK3E transformation assays, KLF4 was shown to signal through non-canonical Notch pathways to induce foci formation. ${ }^{10}$ Expanding on that observation, we demonstrate here that KLF4 can transcriptionally activate Notch1 target genes with no substantial impact on Notch ligands or processing. Furthermore, Notch and KLF4 have multiple overlapping genes as determined by ChIP-seq. While the exact nature of how KLF4 activates Notch target genes requires further investigation, in this context KLF5 had to significant alterations on Notch signaling.

KLF4 and KLF5 demonstrate shared, opposing, and distinct functions in breast cancer. This diverse and interesting relationship has informs future experiments with these genes, possibly providing additional insight by studying both KLFs in tandem where individual studies may be incomplete. On a larger scale, these studies stress the importance of the molecular network of the cell and how our experiments relate to the genetic portrait of patient tumors. By striving to more accurately recapitulate in vivo conditions, we increase the likelihood of describing how these molecules actually impact the disease process.

\section{Reference List}

1 Jiang J, Chan,YS, Loh,YH, Cai,J, Tong,GQ, Lim,CA et al. A core Klf circuitry regulates self-renewal of embryonic stem cells. Nat Cell Biol 2008; 10: 353-360.

2 Rowland BD, Peeper,DS. KLF4, p21 and context-dependent opposing forces in cancer. Nat Rev Cancer 2006; 6: 11-23. 
3 Rowland BD, Bernards,R, Peeper,DS. The KLF4 tumour suppressor is a transcriptional repressor of p53 that acts as a context-dependent oncogene. Nat Cell Biol 2005; 7: 1074-1082.

4 Ghaleb AM, Nandan,MO, Chanchevalap,S, Dalton,WB, Hisamuddin,IM, Yang,VW. Kruppel-like factors 4 and 5: the yin and yang regulators of cellular proliferation. Cell Res 2005; 15: 92-96.

5 McConnell BB, Ghaleb,AM, Nandan,MO, Yang,VW. The diverse functions of Kruppellike factors 4 and 5 in epithelial biology and pathobiology. Bioessays 2007; 29: 549-557.

6 Ghaleb AM, McConnell,BB, Nandan,MO, Katz,JP, Kaestner,KH, Yang,VW. Haploinsufficiency of Kruppel-like factor 4 promotes adenomatous polyposis coli dependent intestinal tumorigenesis. Cancer Res 2007; 67: 7147-7154.

7 McConnell BB, Bialkowska,AB, Nandan,MO, Ghaleb,AM, Gordon,FJ, Yang,VW. Haploinsufficiency of Kruppel-like factor 5 rescues the tumor-initiating effect of the Apc(Min) mutation in the intestine. Cancer Res 2009; 69: 4125-4133.

8 Kenchegowda D, Swamynathan,S, Gupta,D, Wan,H, Whitsett,J, Swamynathan,SK. Conditional disruption of mouse Klf5 results in defective eyelids with malformed meibomian glands, abnormal cornea and loss of conjunctival goblet cells. Dev Biol 2011; 356: 5-18.

9 Swamynathan SK, Katz,JP, Kaestner,KH, Ashery-Padan,R, Crawford,MA, Piatigorsky,J. Conditional deletion of the mouse Klf4 gene results in corneal epithelial fragility, stromal edema, and loss of conjunctival goblet cells. Mol Cell Biol 2007; 27: 182194.

10 Liu Z, Teng,L, Bailey,SK, Frost,AR, Bland,KI, Lobuglio,AF et al. Epithelial transformation by KLF4 requires Notch1 but not canonical Notch1 signaling. Cancer Biol Ther 2009; 8: 1840-1851.

$11 \mathrm{Yu}$ F, Li,J, Chen,H, Fu,J, Ray,S, Huang,S et al. Kruppel-like factor 4 (KLF4) is required for maintenance of breast cancer stem cells and for cell migration and invasion. Oncogene 2011; 30: 2161-2172. 
12 Lin CC, Liu,LZ, Addison,JB, Ivanov,AV, Ruppert,JM. A KLF4-miRNA-206 autoregulatory feedback loop can promote or inhibit protein translation depending upon cell context. Mol Cell Biol 2011; 31: 2513-2527.

13 Chen X, Xu,H, Yuan,P, Fang,F, Huss,M, Vega,VB et al. Integration of external signaling pathways with the core transcriptional network in embryonic stem cells. Cell 2008; 133: 1106-1117.

14 Li Y, Hibbs,MA, Gard,AL, Shylo,NA, Yun,K. Genome-wide analysis of N1ICD/RBPJ targets in vivo reveals direct transcriptional regulation of Wnt, SHH, and hippo pathway effectors by Notch1. Stem Cells 2012; 30: 741-752.

15 Yoon HS, Yang,VW. Requirement of Kruppel-like factor 4 in preventing entry into mitosis following DNA damage. J Biol Chem 2004; 279: 5035-5041.

16 Nandan MO, Chanchevalap,S, Dalton,WB, Yang,VW. Kruppel-like factor 5 promotes mitosis by activating the cyclin B1/Cdc2 complex during oncogenic Ras-mediated transformation. FEBS Lett 2005; 579: 4757-4762.

17 Zheng H, Pritchard,DM, Yang,X, Bennett,E, Liu,G, Liu,C et al. KLF4 gene expression is inhibited by the notch signaling pathway that controls goblet cell differentiation in mouse gastrointestinal tract. Am J Physiol Gastrointest Liver Physiol 2009; 296: G490-G498.

18 Segre JA, Bauer,C, Fuchs,E. Klf4 is a transcription factor required for establishing the barrier function of the skin. Nat Genet 1999; 22: 356-360.

19 Katz JP, Perreault,N, Goldstein,BG, Lee,CS, Labosky,PA, Yang,VW et al. The zinc-finger transcription factor Klf4 is required for terminal differentiation of goblet cells in the colon. Development 2002; 129: 2619-2628.

20 Yori JL, Johnson,E, Zhou,G, Jain,MK, Keri,RA. Kruppel-like factor 4 inhibits epithelialto-mesenchymal transition through regulation of E-cadherin gene expression. $J$ Biol Chem 2010; 285: 16854-16863.

21 Tiwari N, Meyer-Schaller,N, Arnold,P, Antoniadis,H, Pachkov,M, van Nimwegen,E et al. Klf4 is a transcriptional regulator of genes critical for EMT, including Jnk1 (Mapk8). PLoS One 2013; 8: e57329-. 
22 Foster KW, Ren,S, Louro,ID, Lobo-Ruppert,SM, McKie-Bell,P, Grizzle,W et al. Oncogene expression cloning by retroviral transduction of adenovirus E1A-immortalized rat kidney RK3E cells: transformation of a host with epithelial features by c-MYC and the zinc finger protein GKLF. Cell Growth Differ 1999; 10: 423-434.

23 Takahashi K, Yamanaka,S. Induction of pluripotent stem cells from mouse embryonic and adult fibroblast cultures by defined factors. Cell 2006; 126: 663-676.

24 Yori JL, Seachrist,DD, Johnson,E, Lozada,KL, Abdul-Karim,FW, Chodosh,LA et al. Kruppel-like factor 4 inhibits tumorigenic progression and metastasis in a mouse model of breast cancer. Neoplasia 2011; 13: 601-610.

25 Yu F, Shi,Y, Wang,J, Li,J, Fan,D, Ai,W. Deficiency of Kruppel-like factor KLF4 in mammary tumor cells inhibits tumor growth and pulmonary metastasis and is accompanied by compromised recruitment of myeloid-derived suppressor cells. Int J Cancer 2013; 133: 2872-2883.

26 Mani SA, Guo,W, Liao,MJ, Eaton,EN, Ayyanan,A, Zhou,AY et al. The epithelialmesenchymal transition generates cells with properties of stem cells. Cell 2008; 133: 704-715. 


\section{Figure legends}

Fig. 1. Regulation of cell proliferation by endogenous Klf4 and Klf5 in mouse C3(1) TAg M6 cells. A) The indicated shRNA vectors were stably expressed in M6 cells. A) Whole cell extracts of the same M6 derived cell lines were analyzed by immunoblot. B) $1.0 \times 10^{3}$ cells were plated on day 0 and cell numbers were determined over a three day period (N=5; bars, SD) $\mathrm{C}$ ) Ccnb1 was analyzed in M6 cells expressing the indicated $\operatorname{shRNA}(\mathrm{s}) . * \mathrm{p}^{*}<0.05 ; * *, \mathrm{p}<0.01 ; * * *$, $\mathrm{p}<0.001$.

Fig. 2. Non-redundant functions of the core KLFs in breast cancer. A) Western Blot analysis of KLF2, KLF4, and KLF5 in MDA-MB-231 cells expressing shRNA to KLF4. Anoikis in KLF4-depleted cells as determined by trypan blue exclusion. B) Whole cell lysate of MDA-MB231 KLF4 knockdown cells rescued with vector control, KLF2, KLF4, or KLF5. Anoikis sensitivity of the aforementioned cells as determined by trypan blue exclusion. C) Quantitation of anoikis induced cell death via an independent method using fluorescent microscopy. D) MDA-MB-231 cells expressing non-targeting control shRNA were analyzed for CD44 and CD24 expression by flow cytometry. Histograph of CD44 expression in the CD $44^{+} / \mathrm{CD}^{2} 4^{-}$ population with markers indicating the highest quartile of expressing cells. Using this marker, the CD44 expression in the $\mathrm{CD}_{4} 4^{+} / \mathrm{CD} 24^{-}$population of the knockdown-rescue cells was assessed, comparing each sample to the levels in the cells expressing non-targeting control. These results were quantitated in three independent experiments. 
Figure 3. KLF4 functions in canonical Notch1 signaling. A) Target genes of KLF4 and Notch1 were determined by ChIP-seq. ${ }^{13,14}$ For our purposes, we used a gene association score of $\geq 0.9$ for KLF4 and a $\mathrm{p}$-value $\leq 1.0 \times 10^{-4}$ for N1ICD to determine targeted genes. Overlapping target genes from the respective datasets were identified using the statistical program $\mathrm{R}$. B) Putative KLF4 and RBP-Jк binding sites were identified on the promoter of the N1ICD target gene Hes5 using JASPAR core. Identification of direct binding of KLF4 to the Hes5 promoter was assessed via ChIP and qRT-PCR for the indicated regions. C) The transcript levels of Notch1 targeted genes in HMLE cells expressing KLF4 and/or KLF5 as determined by qRTPCR. D) The activity of the Hes1 promoter in HMLE cells expressing KLF4 and/or KLF5 determined by luciferase reporter activity. 
Table 1. KEGG analysis of combined targets of N1ICD/RBPJ and KLF4 for cancer-relevant pathways (47 genes, $p=4.46 \mathrm{e}-24)$

\begin{tabular}{|lrc|}
\hline & $n$ & Genes \\
\hline Wnt Pathway & 6 & Wnt7b, Fzd2, Fzd8, Ctnna1, Ctnnb1, Gsk1b \\
Ras and effector pathways & 12 & Kras, Erbb2, Sos1, Ralgds, Rac3, Rac1, Cdc42, Mapk9, Pik3r1, Pik3cb, Tpr, Tpm3 \\
Cytokine and Growth receptors & 3 & Erbb2, Igf1r, Fgfr3 \\
Apoptosis & 8 & Bcl2l1, Traf4, Casp9, Msh6, Msh2, Nfkb1, Bid, Nfkbia \\
ECM-Receptor interaction & 3 & Itgb1, Lamc1, Lamb2 \\
Proliferation & 4 & Skp2, CDK2, CDK4, Rb1 \\
Bcr-Abl & 3 & Bcr, Abl1, Crk \\
Other pathways & 8 & Hsp90ab1, Hsp90b1, Smad3, Smad2, Jup, Slc2a1, Jak1, Gli3 \\
\hline
\end{tabular}

Table 1. KEGG pathway analysis of the shared targets for N1ICD and KLF4 for cancer-relevant pathways. Pathway analysis was performed using WebGestalt. 
A
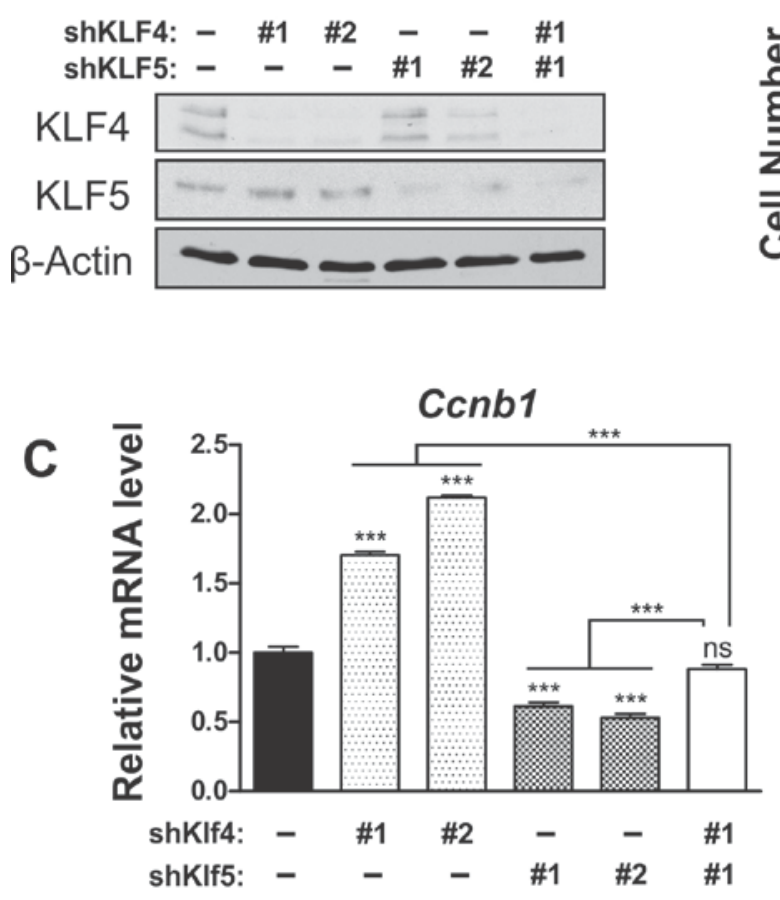

B

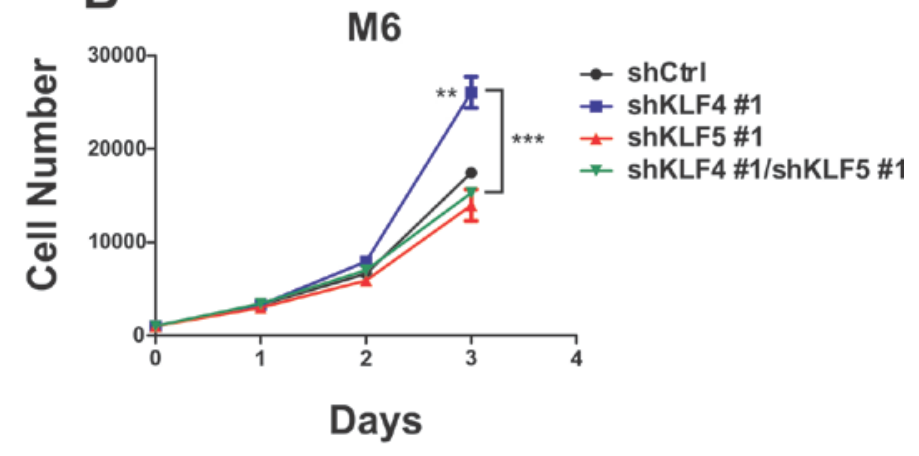

Fig. 1 
A

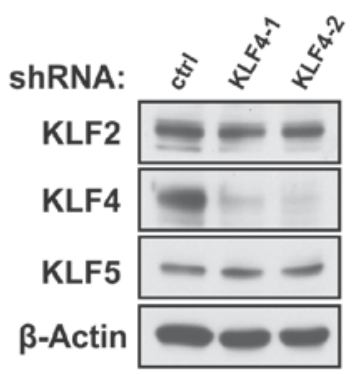

B

Rescue: Vector KLF2 KLF4 KLF5
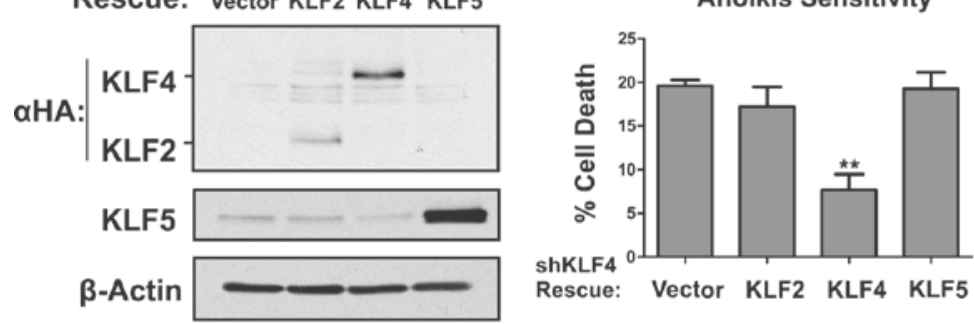

D
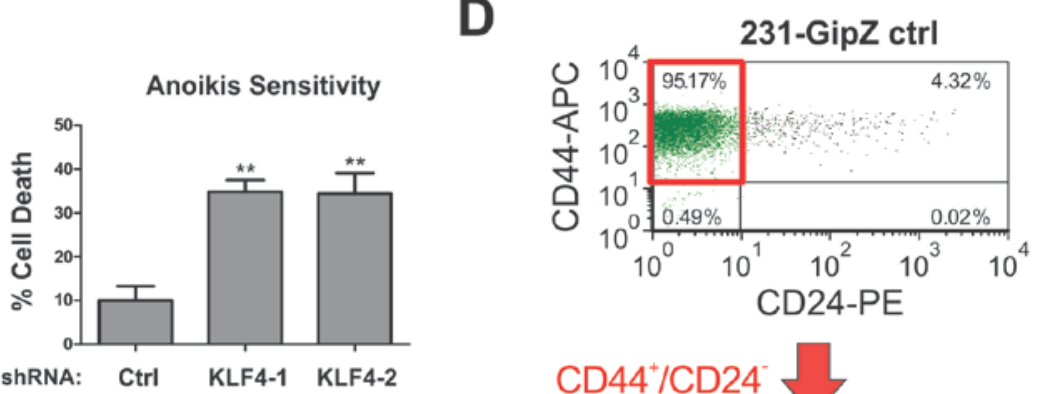

$\mathrm{CD}_{4} 4^{+} / \mathrm{CD} 24$
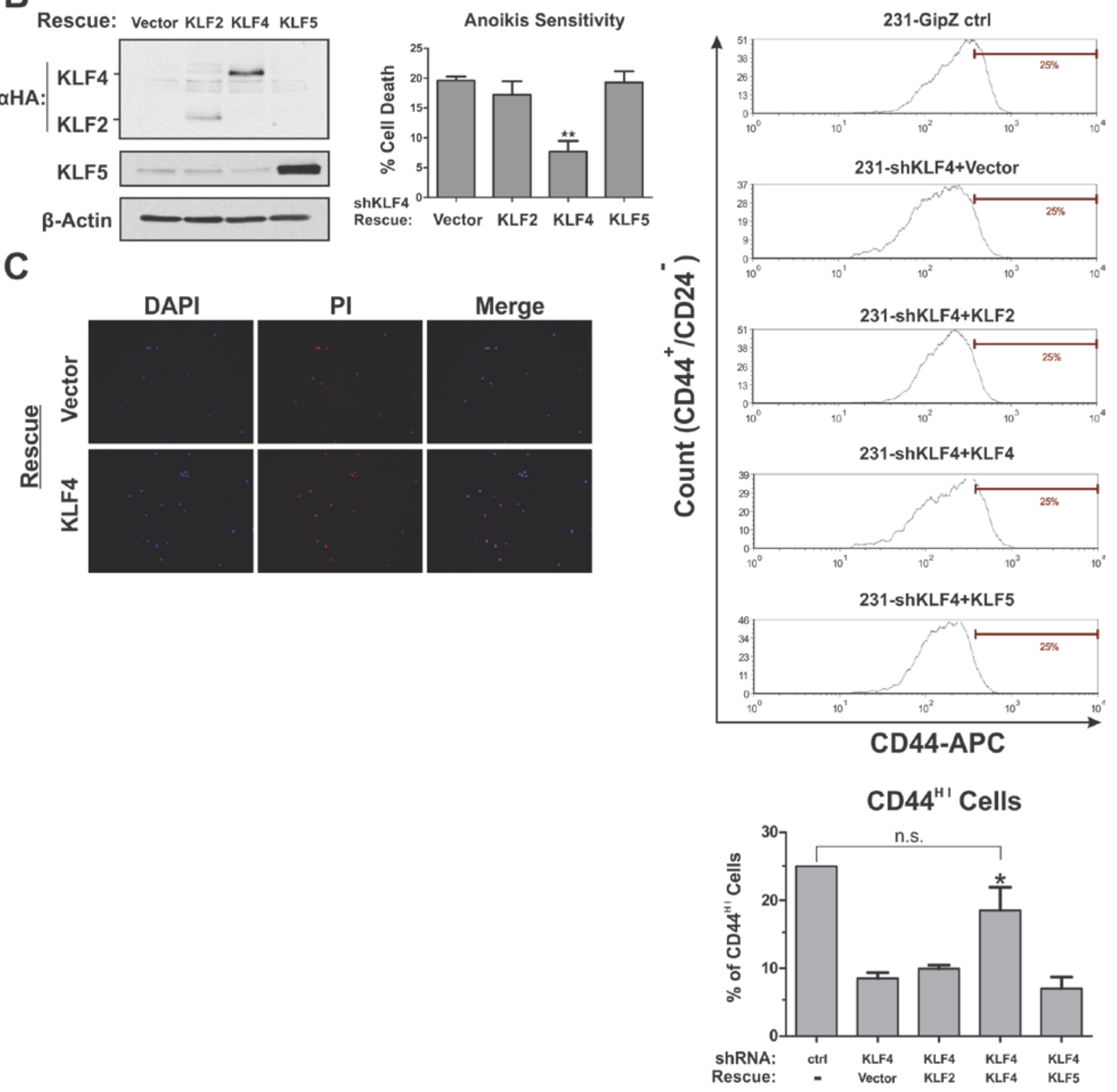

Fig2 

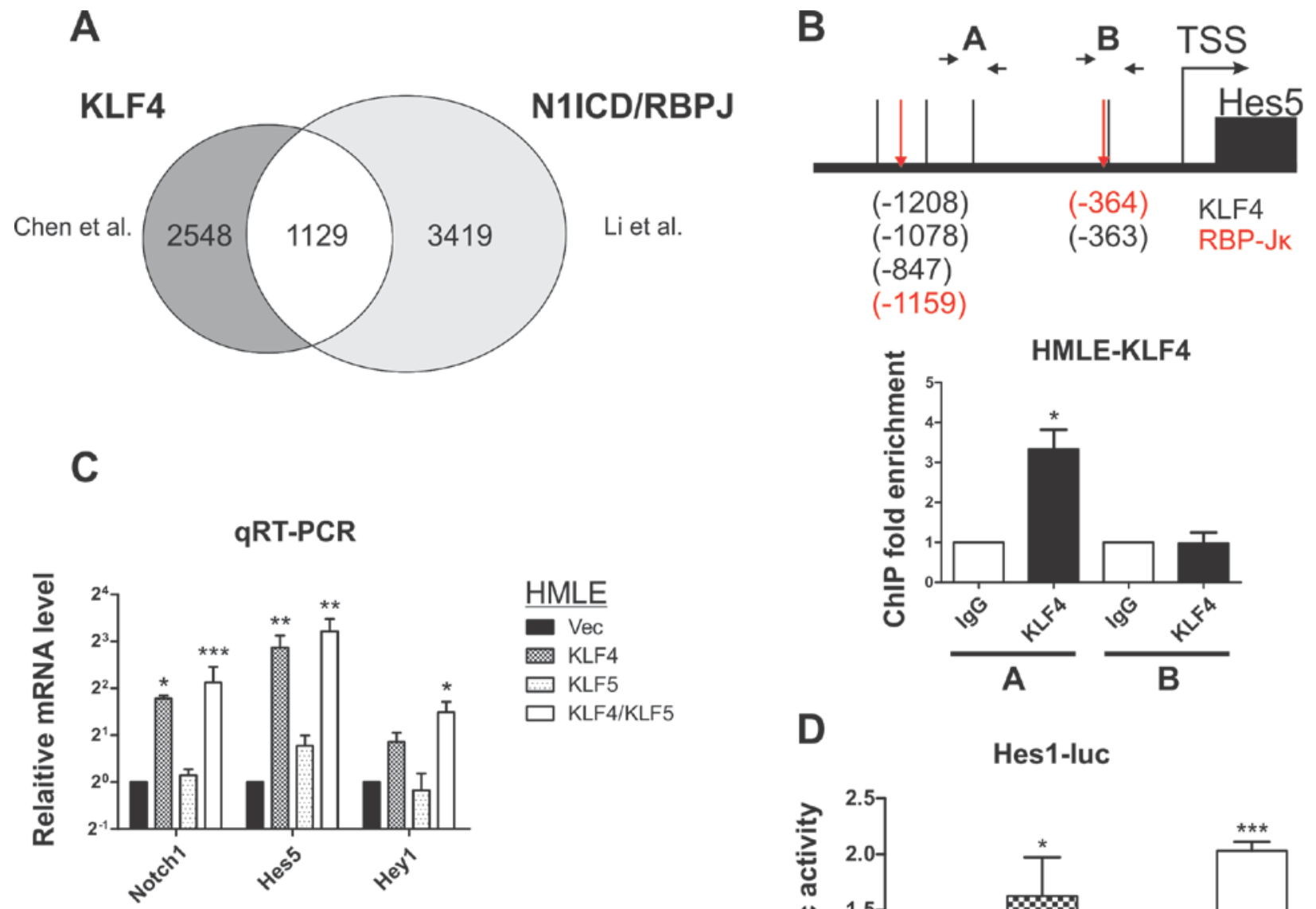

Fig 3
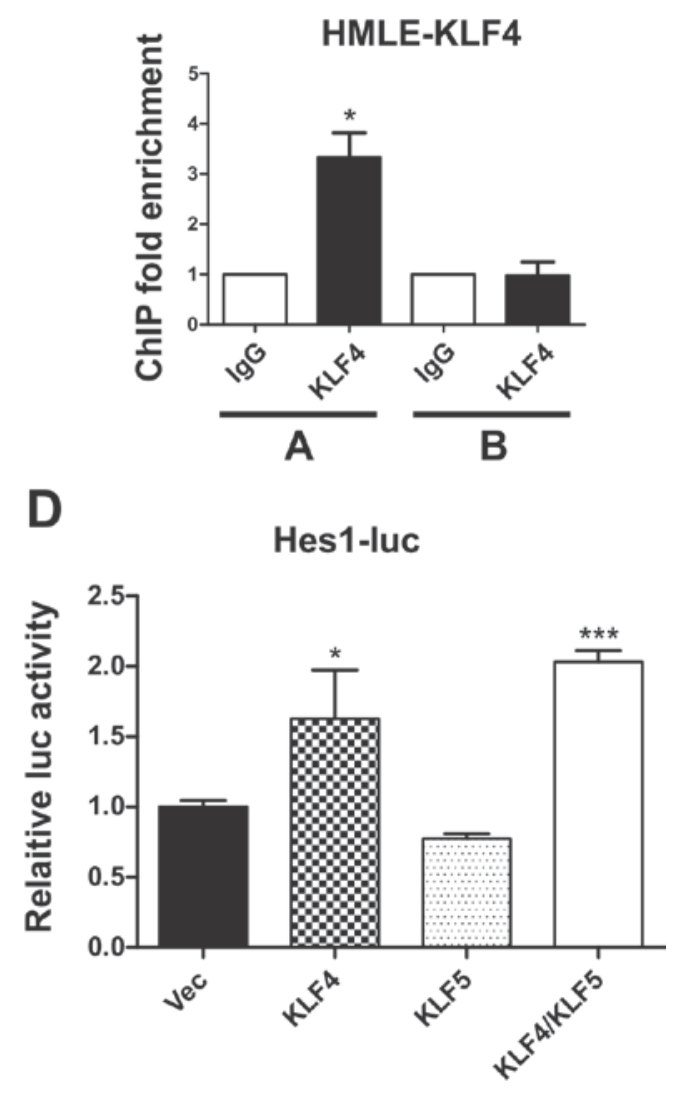


\section{Primers for QPCR}

Primer (5'-3')

Gene

GTGGTAAGGTTTCTCGCCTGT

MusKLF4_Reverse

GGAAAAGAACAGCCACCCAC

MusKLF4_Forward

GGTCTACCACTGAGGCACTG

MusKLF5_Reverse

GAGCTGGTCCAGACAAGATGT

MusKLF5_Forward

GCACATCACTCAGAATTTCAATGG

musRPLP0-

Reverse

GGACCCGAGAAGACCTCCTT

musRPLP0-

Forward

AAGGTGCCTGTGTGTGAACC

musCyclinB1_F

GTCAGCCCCATCATCTGCG

musCyclinB1_R

GGTGAGACCTGCCTGAATG

hsNTCH1-Forward

GTTGGGGTCCTGGC ATC

hsNTCH1-Reverse

GCTTCAGCTGCTCGATGCT

HsHes5_Reverse

TCAGCCCCAAAGAGAAAAAC

HsHes5_Forward

CGAAATCCCAAACTCCGATA

HsHey1_Reverse 
HA-tag PCR cloning primers for KLF2

attggatccggega gtctgacatgtacccctacgacgtgccegactacgccgcgctgagtgaacccatc HAKLF2-Forward attgaattcctacatgtgccgtttcatgt

HAKLF2-Reverse 


\section{Chapter 4: Summary discussion}

Kruppel-like factors (KLFs) are central regulators of cell fate that can have profound effects on the cancer phenotype, yet their relevant effector pathways remain poorly understood. Examination of these factors may allow for an improved understanding of how KLF signaling relates to clinically significant parameters such as drug resistance. Indeed recent results implicate these factors as critical mediators to adaptive responses following targeted therapies. ${ }^{1}$ KLF4 and KLF5 (KLF4/5) have been identified as coexpressed factors that cooperate to mediate drug resistance, tumorigenesis, and distant metastasis-free survival in breast cancer. ${ }^{1}$ However, several previous studies on these individual factors suggest antagonistic functions between the two KLFs. $^{2-4}$ Therefore, we sought gain insight into the findings in breast cancer through their documented relationships in embryonic stem (ES) cells, tissue development, cardiovasculature, and other pathological conditions.

\section{The role of KLFs in pluripotency}

KLFs illustrate diverse interactions at the earliest stages of human life, as evident by divergent and convergent signaling. In ES cells, differentiation can be induced by triple knockdown of KLF2/KLF4/KLF5, homozygous ablation of Klf5, or withdrawal of leukemia inhibitory factor (LIF) which is accompanied by a reduction in KLF expression. ${ }^{5}$ Furthermore, introducing ectopic KLF2, KLF4, or KLF5 into the differentiated ES cells accomplished by any of these aforementioned methods is sufficient to restore pluripotency. ${ }^{5}$

Although these overlapping abilities are suggestive of redundancy between these factors, KLFs can exert numerous distinct effects within ES cells. Chromatin immunoprecipitation followed by next-generation sequencing of KLF4/5 has identified roughly equal numbers of shared and distinct targets genes between the two factors. ${ }^{6}$ Although KLF4/5 both suppress 
differentiation, each inhibits differentiation to mutually exclusive lineages, the endoderm and mesoderm respectively. Likewise, KLF5 reduction can impact at least 26 different genes in ES cells that are unaffected KLF2 or KLF4. ${ }^{5}$ While the ability of the KLFs to maintain the ES cell phenotype in the absence of LIF is shared, the potency at which this is accomplished can vary between them with KLF2 as the most effective, KLF4 intermediate, and KLF5 as the least efficacious. ${ }^{5}$ Although KLF4 and KLF5 are elevated in the stem compartments of adult tissues, KLF2 and KLF4 are far more efficient in somatic reprogramming compared to KLF5. ${ }^{5}$

Furthermore in breast cancer, only KLF4, not KLF2 or KLF5, can rescue defects in cancer stem

cell properties as a result of KLF4 depletion.(Chapter 3) These observations are possibly a result of distinct effects on Notch pathway activity by KLF4.

These reports support the premise that KLFs 2,4 and 5 have common functions, likely due to evolutionary conservation in KLF structure and their binding sites at certain loci. However, the differences in these factors contribute to the extent that each can participate in these processes, as well as allow for unique targets and roles among the KLFs.

\section{Conserved and distinct functions of KLF4 and 5 in development}

Although the relationships between these KLFs may be attributed to evolutionary remnants, this does not seem to be the case. KLF4/5 are frequently coexpressed in developing tissues and stem cell compartments in both normal and pathological settings. ${ }^{3,7-12}$ Although mutually present in the same developing structures, often times they localize to distinct compartments. KLF4 is commonly associated with post-mitotic, growth-arrested epithelium while KLF5 is highly expressed in proliferating cells. ${ }^{2,9}$ Furthermore, KLF4/5 can target several cell cycle regulatory genes such as p21 ${ }^{\text {Waf1/Cip1 }}, \mathrm{p} 15, \mathrm{p} 27, \mathrm{CCNB} 1, \mathrm{CCND} 1$, and CCNE1 in an opposite manner. ${ }^{2}$ Moreover, KLF4/5 can exhibit mutual antagonism on their respective 
promoters. ${ }^{13}$ These reports suggest that an antagonistic relationship exists between KLF4/5 in somatic tissues.

However, there appears to be additional complexity to the KLF4/KLF5 story. Conditional deletion of KIf 4 or Klf5 in the surface ectoderm of the eye reveal similar developmental deficits. $^{8,12}$ Genetic ablation of either KLF results in dramatically reduced goblet cell number and function, suggesting convergent signaling. Similar effects are observed in gut maturation as well, where intestinal specific knockouts of Klf4 or KIf5 resulted in goblet cell deficits. ${ }^{14-16}$ Other reports on the function of KLF4/5 in the intestines would advocate opposing influences, yet clearly they can exhibit shared activities. ${ }^{3}$ Illustrated here is the previously suggested notion that the role of the KLFs are highly dependent on context, a theme which can seemingly be extended to include the relationship between these factors as well. ${ }^{17,18}$

\section{Crucial effects of the KLFs in the stress response of the cardiovasculature}

To further understand the basis of how KLF4/5 impact the cellular response to stress, we reviewed their role in the cardiovascular system, where both factors have been extensively studied. Although KLF4/5 are highly expressed in the embryonic vasculature, typically levels of these factors are low in normal adult vascular smooth muscle cells (SMCs). ${ }^{19}$ However in these cells, KLF4/5 are induced in response to various noxious stimuli. ${ }^{19}$ Additionally, doxorubicin and oxidant exposure to cardiac myocytes can also stimulate these factors. ${ }^{10}$ Injury to the vasculature, for example caused by shear stress, coronary atherosclerosis, vein graft hyperplasia, oxidized phospholipids, and/or ischemia, can trigger an adaptive response in SMCs prompting dedifferentiation and proliferation. ${ }^{19-23}$ In conditional $\mathrm{Klf} 4^{-/-}$mice, the damage related suppression of SMC differentiation markers is delayed compared to wildtype mice. ${ }^{22}$ Similarly, the oxidized phospholipid POVPC requires the activity of KLF4 and other factors to promote 
SMC dedifferentiation. ${ }^{21}$ Although the neointima of conditional $\mathrm{Klf}^{-/-}$mice exhibit elevated cell growth in response injury, these mice are more vulnerable to vascular insults, indicating defects in repair due to impaired dedifferentiation.

On the other hand, KLF5 mediates cardiovascular stress response though enhanced proliferation. Mice heterozygous for Klf5 show reduced response to cardiovascular insults, such as arterial polyethylene cuffs, characterized by thinner medial and intimal layers. ${ }^{19} \mathrm{By}$ comparison, wildtype mice exhibited arterial layers thick with granulation tissue, extracellular matrix, and angiogenesis in response to the same injuries. Furthermore, KLF5 overexpression upregulates vascular injury response factors such as iNOS, PAI-1, PDGF-A and VEGFRs. ${ }^{20,24-26}$ Moreover, KLF5 expression confers increased cell survival in atherosclerotic vascular lesions. ${ }^{27}$ KLF5, as well as KLF4, is induced by endothelin-1 in neonatal rat myocytes. Endothelins are the most potent known vasoconstrictors and have important roles in pressure regulation of the vasculature, as well as implications in cardiovascular disease. ${ }^{28}$ Interestingly, microRNA-145 (miR-145) is highly expressed in differentiated adult SMCs and dramatically downregulated in atherosclerotic lesions. ${ }^{29}$ miR-145 directly suppresses KLF4/5 translation and its depletion is likely represents a sensor/effector system tailored to mediate damage in the vasculature.

These results highlight the need for both KLF4/5 activity in the injury response program of vascular SMCs. Even though conditional $\mathrm{Klf}^{-/-}$mice exhibit greater degrees of insult-related proliferation, the lack of proper dedifferentiation ultimately produces an insufficient adaptive response. Likewise, it is likely that the dedifferentiation program of SMCs in Klf5 ${ }^{+/}$mice is intact or possibly enhanced, yet without adequate proliferation the response to damage is incomplete. 


\section{Adaptive and survival functions of the KLFs in response to noxious stimuli}

In addition to their role in the vasculature, KLF4/5 mediate survival to noxious stimuli in a variety of cellular and physiological contexts. KLF4 expression is elevated in wound healing

keratinocytes and moreover, the induction of KLF4 by heat stress is critical to cell viability. ${ }^{30,31}$ Furthermore, KLF4 is vital in protecting neurons and fibroblasts from oxidative damage. ${ }^{32,33}$ Several studies demonstrate an important role for KLF4 in cellular survival following $\gamma$ radiation. ${ }^{34-36}$ In gut epithelium, KLF4 attenuates radiation-induced damage and cell death. ${ }^{36}$ Mechanistically, the radioprotective effects of KLF4 are in part attributed to p53 signaling. ${ }^{34,35}$ The p53-dependent induction of KLF4 causes growth arrest through p21 ${ }^{\text {Waf1/Cip } 1}$, where the cessation of the cell cycle allows for adequate DNA repair. Without KLF4, cells prematurely entered mitosis resulting in apoptosis. Additionally, KLF4 also inhibits pro-apoptotic Bax expression in response to $\gamma$-radiation. ${ }^{35}$ Taken together, KLF4 is capable of protecting cells from a variety of harmful stimuli and may be associated with a conserved cellular response to toxic insults.

Similar to KLF4, KLF5 participates in the adaptive response to external stressors in several organ systems. In the gut, KLF5 enhances protection and recovery to dextran sulfate sodium-induced injury. ${ }^{37}$ Likewise, KLF5 is a key mediator in the colonic response to pathogenic bacterial infection. ${ }^{38}$ In addition to the cardiovasculature, KLF5 promotes survival in the pulmonary blood vessels as well. KLF5 contributes to pulmonary artery smooth muscle cell survival in hypertensive settings, and its expression correlates with increased levels of activated survivin in experimental models of congenital diaphragmatic hernia. ${ }^{39,40}$ In fact, KLF5 enhances anti-apoptotic activity through survivin in a variety of other contexts, such as pancreatic, ovarian, and small cell lung cancers as well as acute lymphoblastic leukemia. ${ }^{41-44}$ KLF5 modulates 
apoptotic signaling to enhance survival in other settings as well. In response to intracerebral hemorrhage, KLF5 protects against neuronal apoptosis by suppressing pro-apoptotic Bad activation. ${ }^{45}$ In summary, the consistent involvement of KLF4/5 within the stress response of multiple systems argue that these factors are conserved responders to damage of cells and tissues.

\section{Role of KLF4/5 in breast cancer cell survival}

As cell fate regulatory factors that impact both stress response signaling and cell survival, these factors would seem attractive as mediators of tumorigenesis. KLF5 has typically been implicated as a protumorigenic factor, including breast, bladder, colon, lung, gastric, and HPV-associated cancers, and is often associated with the promotion of cell proliferation. ${ }^{41,46-51}$ On the other hand, KLF5 is linked to tumor suppressor activities in prostate cancers and esophageal squamous cell carcinomas. $^{52-55}$ KLF4 can elicit dual effects on tumor progression, even within the same tumor type. For example, KLF4 has been reported to inhibit tumor progression and/or malignant properties in a variety of contexts, including neuroblastoma, leukemia, pancreatic, lung, and colon cancers. ${ }^{14,56-63}$ Conversely, adverse impact on clinical outcome and/or protumorigenic effects in functional assays have been reported for KLF4 in breast, skin, and colon cancers as well as others. ${ }^{64-72}$

Some of these disparate conclusions may be attributed to differences in methodological approaches, such as manipulation of the endogenous factor versus expression of exogenous transgenes. For KLF4 in particular, these two different methodologic approaches can yield discordant results, as demonstrated by the on-off mode by which KLF4 maintains miR21 transcription to promote RAS-ERK signaling in TNBCs. ${ }^{73}$ In this case, endogenous KLF4 is quite sufficient for high level miR-21 expression, and further elevations of KLF4 as with 
exogenous expression results in little or no additional miR-21 levels. Consequently, it is possible that phenotypes resulting from enforced expression may not reflect the activity of the endogenous factor.

In addition, individual manipulations of KLF4 are often attributed tumor suppressive activities through KLF4's growth-inhibitory effects. ${ }^{74,75}$ Frequently this involves greatly overexpressing KLF4, to the point where cell division is markedly impacted. Such profound effects on the cell cycle are not typical of KLF4-depletion in models of breast cancer, and would likely mask any contributions to other cellular processes, for example cell survival. Attempts to infer the function of a gene through supraphysiologic expression can be limited, as it can convey phenotypes that greatly surpass capabilities of the endogenous protein, possibly producing artifacts. Furthermore, as illustrated in Chapter 3, the coexpression of KLF4/5 ameliorates the cell cycle effects of the two genes. Consistent with these observations, KLF4 expression has not been linked to pathological markers indicating slow growing tumors in patients (e.g. KI67). ${ }^{64}$ Therefore, many of these reports on KLF4 may be uninformative due to the failure to appreciate the effect of coexpressed KLF5, and/or the reliance on overexpression approaches.

\section{Role of KLF4/5 in therapeutic resistance}

It seems common to presume that effective cancer therapies will ultimately need to target the very same pathways that are genetically altered during tumor progression. These pathways include well established targets such as K-RAS, p53, c-MYC and others. Consistent with the absence of frequent genetic alteration of either KLF4 or KLF5 in human tumors, our studies appear to support a model in which KLF4/5 are not critical to tumor pathogenesis, but instead play a critical role in the response to targeted therapy, possibly reflecting a normal function of these factors in the stress response within normal epithelial cells. Consequently, drug resistance 
in cancer may be "inherited" from the tumor precursor cell rather than selected for through clonal evolution during tumor development.

In the absence of any overt cell stressor, the overall effect of endogenous KLF4 in human breast cancer cells is quite subtle.(Manuscript in press) Knockdown studies in human breast tumor lines revealed a prosurvival effect that was critical for xenograft tumor initiation in athymic mice. However KLF4 suppression has no effect on tumor initiation in NOD/SCID/ $\gamma$ mice, which contain additional immunodeficiencies and are more permissive. Indeed in both genetic backgrounds the tumor growth rate of successfully initiated lesions was unaffected by suppression of endogenous KLF4.

In fact, most studies have examined the function of KLF4/5 in relatively permissive contexts, such as in immunocompromised mice. In contrast, tumors in human patients are subjected to incredible stresses including the inflammatory response, chemotherapy, and radiotherapy. As stress response factors, examining how KLF4/KLF5 influence the cellular response to these noxious stimuli may be the more informative approach to understanding their clinical relevance.

In breast cancer, KLF4/5 expression have been associated with poor patient outcome. ${ }^{1,47,64,76}$ Furthermore, in a small breast cancer patient cohort KLF4 expression was independently prognostic for pathologic complete response to neoadjuvant chemotherapy. ${ }^{77}$ Moreover, KLF4 via miR-206 signaling promotes breast cancer stem cell features ultimately conveying enhanced cell survival, as represented by increased tumor initiation, anoikis resistance, and reduced response to cytotoxic drugs. In addition to cytotoxic chemotherapies, KLF4 also mediates resistance to MEK inhibition through receptor tyrosine kinase reprogramming (i.e., PDGFR $\beta$ ).(Manuscript in submission) ${ }^{78}$ 
Similarly, KLF5 has been linked to chemoresistance and cancer stem cell characteristics in other malignancies as well. In ovarian cancer, KLF5 conferred increased resistance to chemotherapies attributed to elevated survivin levels. ${ }^{43}$ Additionally, depletion of KLF5 increased colon cancer cells sensitivity to DNA damaging agents through activated Pim1. ${ }^{79}$ When KLF4/5 were examined in tandem, both factors were observed to be selectively prognostic within the breast cancer HER2-enriched subtype and to facilitate the therapeutic response to HER2-inhibition through the expression of anti-apoptotic factors MCL1 and BCL-XL. ${ }^{1}$ In this setting, individual examination of KLF4 or KLF5 alone gave modest results, however manipulation of both factors was much more effective. Therefore, whenever applicable it may be more informative to study KLF4/5 together.

\section{Targeting KLF4/5 and their effectors}

Although KLF4 has multiple effectors to impact cell survival, both KLF4/5 regulate components of the intrinsic pathway of apoptosis, highlighting an attractive node to target therapeutically. Currently there are two major small molecule inhibitors that target the BCL2 family anti-apoptotic factors under clinical investigation. Obatoclax (GX15-070) and Navitoclax (ABT-263) are BH3-mimetics which disrupt BCL2 family member protein-protein interactions, thereby enabling enhanced pro-apoptotic signaling. Where Obatoclax broadly inhibits all the anti-apoptotic BCL2 family members with a reduced potency, Navitoclax has a high affinity for BCL-2, -XL, and $-\mathrm{W} .{ }^{80}$ Combined, these drugs are involved in 42 active/completed Phase I/II clinical trials for various malignancies, however their efficacy in breast cancer patients has yet to be explored.(clinicaltrials.gov) Although these drugs are well-tolerated in patients, preliminary data suggests they have limited utility as single-agents, advocating consideration for combination therapy instead. Such strategies could be employed in HER2-enriched breast cancer, possibly 
enhancing the efficacy of current regimens and/or reducing the reliance upon cytotoxic chemotherapies.

Therapeutically targeting transcription factors can be difficult, however there many strategies currently under investigation. High-throughput screening identified the small molecule ML264 as a selective inhibitor of KLF5 expression, however its efficacy in vivo has not yet been extensively characterized. ${ }^{81}$ Alternatively, there have been monumental efforts to provide antisense or gene-silencing modalities in patient care. Selectively impacting the gene-expression within a diseased tissue could offer enormous clinical benefits in wide variety of pathological settings. While this field is rapidly advancing, current methods of delivery (e.g., liposome-based nanoparticles) yield inadequate specificity and efficacy. However as these vehicles improve, it is possible that KLF4, KLF5 and/or miR-206 could be selectively depleted in patient tumors, likely increasing their response to other treatments.

Incomplete eradication of cancer cells is the major obstacle to sustained disease-free survival. To successfully combat residual disease, it is essential to understand the cellular reprogramming and adaptive responses to therapies. Here we describe how KLF4/5 are critical mediators to cellular stress, a key feature that is maintained in malignancy to promote cell survival and therapeutic resistance. Effectively targeting KLF4/5 or their respective effectors may attenuate the ability of resistant cells to survive during current treatment regimens, thus increasing the likelihood of long-term cures. 


\section{Reference List}

1 Farrugia MK, Sharma,SB, Lin,CC, McLaughlin,SL, Vanderbilt,DB, Ammer,AG et al. Regulation of anti-apoptotic signaling by Kruppel-like factors 4 and 5 mediates lapatinib resistance in breast cancer. Cell Death Dis 2015; 6: e1699-.

2 Ghaleb AM, Nandan,MO, Chanchevalap,S, Dalton,WB, Hisamuddin,IM, Yang,VW. Kruppel-like factors 4 and 5: the yin and yang regulators of cellular proliferation. Cell Res 2005; 15: 92-96.

3 Ghaleb AM, Yang,VW. The Pathobiology of Kruppel-like Factors in Colorectal Cancer. Curr Colorectal Cancer Rep 2008; 4: 59-64.

4 McConnell BB, Ghaleb,AM, Nandan,MO, Yang,VW. The diverse functions of Kruppellike factors 4 and 5 in epithelial biology and pathobiology. Bioessays 2007; 29: 549-557.

5 Bourillot PY, Savatier,P. Kruppel-like transcription factors and control of pluripotency. BMC Biol 2010; 8: 125-.

6 Aksoy I, Giudice,V, Delahaye,E, Wianny,F, Aubry,M, Mure,M et al. Klf4 and Klf5 differentially inhibit mesoderm and endoderm differentiation in embryonic stem cells. Nat Commun 2014; 5: 3719-.

7 Ohnishi S, Ohnami,S, Laub,F, Aoki,K, Suzuki,K, Kanai,Y et al. Downregulation and growth inhibitory effect of epithelial-type Kruppel-like transcription factor KLF4, but not KLF5, in bladder cancer. Biochem Biophys Res Commun 2003; 308: 251256.

8 Swamynathan SK, Katz,JP, Kaestner,KH, Ashery-Padan,R, Crawford,MA, Piatigorsky,J. Conditional deletion of the mouse Klf4 gene results in corneal epithelial fragility, stromal edema, and loss of conjunctival goblet cells. Mol Cell Biol 2007; 27: 182194.

9 Chen Z, Couble,ML, Mouterfi,N, Magloire,H, Chen,Z, Bleicher,F. Spatial and temporal expression of KLF4 and KLF5 during murine tooth development. Arch Oral Biol 2009; 54: 403-411. 
10 De AA, Piegari,E, Cappetta,D, Marino,L, Filippelli,A, Berrino,L et al. Anthracycline cardiomyopathy is mediated by depletion of the cardiac stem cell pool and is rescued by restoration of progenitor cell function. Circulation 2010; 121: 276292.

11 Gupta PB, Fillmore,CM, Jiang,G, Shapira,SD, Tao,K, Kuperwasser,C et al. Stochastic state transitions give rise to phenotypic equilibrium in populations of cancer cells. Cell 2011; 146: 633-644.

12 Kenchegowda D, Swamynathan,S, Gupta,D, Wan,H, Whitsett,J, Swamynathan,SK. Conditional disruption of mouse Klf5 results in defective eyelids with malformed meibomian glands, abnormal cornea and loss of conjunctival goblet cells. Dev Biol 2011; 356: 5-18.

13 Dang DT, Zhao,W, Mahatan,CS, Geiman,DE, Yang,VW. Opposing effects of Kruppellike factor 4 (gut-enriched Kruppel-like factor) and Kruppel-like factor 5 (intestinal-enriched Kruppel-like factor) on the promoter of the Kruppel-like factor 4 gene. Nucleic Acids Res 2002; 30: 2736-2741.

14 Katz JP, Perreault,N, Goldstein,BG, Lee,CS, Labosky,PA, Yang,VW et al. The zincfinger transcription factor Klf4 is required for terminal differentiation of goblet cells in the colon. Development 2002; 129: 2619-2628.

15 Zheng H, Pritchard,DM, Yang,X, Bennett,E, Liu,G, Liu,C et al. KLF4 gene expression is inhibited by the notch signaling pathway that controls goblet cell differentiation in mouse gastrointestinal tract. Am J Physiol Gastrointest Liver Physiol 2009; 296: G490-G498.

16 Nandan MO, Ghaleb,AM, Liu,Y, Bialkowska,AB, McConnell,BB, Shroyer,KR et al. Inducible intestine-specific deletion of Kruppel-like factor 5 is characterized by a regenerative response in adult mouse colon. Dev Biol 2014; 387: 191-202.

17 Rowland BD, Peeper,DS. KLF4, p21 and context-dependent opposing forces in cancer. Nat Rev Cancer 2006; 6: 11-23.

18 Rowland BD, Bernards,R, Peeper,DS. The KLF4 tumour suppressor is a transcriptional repressor of p53 that acts as a context-dependent oncogene. Nat Cell Biol 2005; 7: 1074-1082. 
19 Suzuki T, Aizawa,K, Matsumura,T, Nagai,R. Vascular implications of the Kruppel-like family of transcription factors. Arterioscler Thromb Vasc Biol 2005; 25: 11351141.

20 Bafford R, Sui,XX, Wang,G, Conte,M. Angiotensin II and tumor necrosis factor-alpha upregulate survivin and Kruppel-like factor 5 in smooth muscle cells: Potential relevance to vein graft hyperplasia. Surgery 2006; 140: 289-296.

21 Yoshida T, Gan,Q, Owens,GK. Kruppel-like factor 4, Elk-1, and histone deacetylases cooperatively suppress smooth muscle cell differentiation markers in response to oxidized phospholipids. Am J Physiol Cell Physiol 2008; 295: C1175-C1182.

22 Yoshida T, Kaestner,KH, Owens,GK. Conditional deletion of Kruppel-like factor 4 delays downregulation of smooth muscle cell differentiation markers but accelerates neointimal formation following vascular injury. Circ Res 2008; 102: 1548-1557.

23 Cullingford TE, Butler,MJ, Marshall,AK, Tham,eL, Sugden,PH, Clerk,A. Differential regulation of Kruppel-like factor family transcription factor expression in neonatal rat cardiac myocytes: effects of endothelin-1, oxidative stress and cytokines. Biochim Biophys Acta 2008; 1783: 1229-1236.

24 Zhang Z, Teng,CT. Phosphorylation of Kruppel-like factor 5 (KLF5/IKLF) at the CBP interaction region enhances its transactivation function. Nucleic Acids Res 2003; 31: 2196-2208.

25 Kawai-Kowase K, Kurabayashi,M, Hoshino,Y, Ohyama,Y, Nagai,R. Transcriptional activation of the zinc finger transcription factor BTEB2 gene by Egr-1 through mitogen-activated protein kinase pathways in vascular smooth muscle cells. Circ Res 1999; 85: 787-795.

26 Gao D, Niu,X, Ning,N, Hao,G. Regulation of angiotensin II-Induced Kruppel-like factor 5 expression in vascular smooth muscle cells. Biol Pharm Bull 2006; 29: 20042008.

27 Suzuki T, Nishi,T, Nagino,T, Sasaki,K, Aizawa,K, Kada,N et al. Functional interaction between the transcription factor Kruppel-like factor 5 and poly(ADP-ribose) polymerase-1 in cardiovascular apoptosis. J Biol Chem 2007; 282: 9895-9901. 
28 Sugden PH. An overview of endothelin signaling in the cardiac myocyte. J Mol Cell Cardiol 2003; 35: 871-886.

29 Rangrez AY, Massy,ZA, Metzinger-Le,M, V, Metzinger,L. miR-143 and miR-145: molecular keys to switch the phenotype of vascular smooth muscle cells. Circ Cardiovasc Genet 2011; 4: 197-205.

30 Liu Y, Wang,J, Yi,Y, Zhang,H, Liu,J, Liu,M et al. Induction of KLF4 in response to heat stress. Cell Stress Chaperones 2006; 11: 379-389.

31 Pedersen TX, Leethanakul,C, Patel,V, Mitola,D, Lund,LR, Dano,K et al. Laser capture microdissection-based in vivo genomic profiling of wound keratinocytes identifies similarities and differences to squamous cell carcinoma. Oncogene 2003; 22: 3964-3976.

32 Su C, Sun,F, Cunningham, RL, Rybalchenko,N, Singh,M. ERK5/KLF4 signaling as a common mediator of the neuroprotective effects of both nerve growth factor and hydrogen peroxide preconditioning. Age (Dordr ) 2014; 36: 9685-.

33 Liu C, La,RS, Hagos,EG. Oxidative DNA damage causes premature senescence in mouse embryonic fibroblasts deficient for Kruppel-like factor 4. Mol Carcinog 2014.

34 Yoon HS, Chen,X, Yang,VW. Kruppel-like factor 4 mediates p53-dependent G1/S cell cycle arrest in response to DNA damage. J Biol Chem 2003; 278: 2101-2105.

35 Ghaleb AM, Katz,JP, Kaestner,KH, Du,JX, Yang,VW. Kruppel-like factor 4 exhibits antiapoptotic activity following gamma-radiation-induced DNA damage. Oncogene 2007; 26: 2365-2373.

36 Talmasov D, Xinjun,Z, Yu,B, Nandan,MO, Bialkowska,AB, Elkarim,E et al. Kruppellike factor 4 is a radioprotective factor for the intestine following gammaradiation-induced gut injury in mice. Am J Physiol Gastrointest Liver Physiol 2015; 308: G121-G138.

37 McConnell BB, Kim,SS, Bialkowska,AB, Yu,K, Sitaraman,SV, Yang,VW. Kruppel-like factor 5 protects against dextran sulfate sodium-induced colonic injury in mice by promoting epithelial repair. Gastroenterology 2011; 140: 540-549. 
38 McConnell BB, Klapproth,JM, Sasaki,M, Nandan,MO, Yang,VW. Kruppel-like factor 5 mediates transmissible murine colonic hyperplasia caused by Citrobacter rodentium infection. Gastroenterology 2008; 134: 1007-1016.

39 Courboulin A, Tremblay,VL, Barrier,M, Meloche,J, Jacob,MH, Chapolard,M et al. Kruppel-like factor 5 contributes to pulmonary artery smooth muscle proliferation and resistance to apoptosis in human pulmonary arterial hypertension. Respir Res 2011; 12: 128-.

40 Hofmann AD, Takahashi,T, Duess,JW, Gosemann,JH, Puri,P. Increased pulmonary vascular expression of Kruppel-like factor 5 and activated survivin in experimental congenital diaphragmatic hernia. Pediatr Surg Int 2014; 30: 11911197.

41 Li X, Liu,X, Xu,Y, Liu,J, Xie,M, Ni,W et al. KLF5 promotes hypoxia-induced survival and inhibits apoptosis in non-small cell lung cancer cells via HIF-1alpha. Int $J$ Oncol 2014; 45: 1507-1514.

42 Zhu N, Gu,L, Findley,HW, Chen,C, Dong,JT, Yang,L et al. KLF5 Interacts with p53 in regulating survivin expression in acute lymphoblastic leukemia. J Biol Chem 2006; 281: 14711-14718.

43 Dong Z, Yang,L, Lai,D. KLF5 strengthens drug resistance of ovarian cancer stem-like cells by regulating survivin expression. Cell Prolif 2013; 46: 425-435.

44 Mori A, Moser,C, Lang,SA, Hackl,C, Gottfried,E, Kreutz,M et al. Up-regulation of Kruppel-like factor 5 in pancreatic cancer is promoted by interleukin-1beta signaling and hypoxia-inducible factor-1alpha. Mol Cancer Res 2009; 7: 13901398.

45 Liu X, Yuan,D, Nie,X, Shen,J, Yan,Y, Zhang,D et al. BTEB2 prevents neuronal apoptosis via promoting bad phosphorylation in rat intracerebral hemorrhage model. J Mol Neurosci 2015; 55: 206-216.

46 Chen C, Benjamin,MS, Sun,X, Otto,KB, Guo,P, Dong,XY et al. KLF5 promotes cell proliferation and tumorigenesis through gene regulation and the TSU-Pr1 human bladder cancer cell line. Int J Cancer 2006; 118: 1346-1355. 
47 Tong D, Czerwenka,K, Heinze,G, Ryffel,M, Schuster,E, Witt,A et al. Expression of KLF5 is a prognostic factor for disease-free survival and overall survival in patients with breast cancer. Clin Cancer Res 2006; 12: 2442-2448.

48 McConnell BB, Bialkowska,AB, Nandan,MO, Ghaleb,AM, Gordon,FJ, Yang,VW. Haploinsufficiency of Kruppel-like factor 5 rescues the tumor-initiating effect of the Apc(Min) mutation in the intestine. Cancer Res 2009; 69: 4125-4133.

49 Nandan MO, Ghaleb,AM, McConnell,BB, Patel,NV, Robine,S, Yang,VW. Kruppel-like factor 5 is a crucial mediator of intestinal tumorigenesis in mice harboring combined ApcMin and KRASV12 mutations. Mol Cancer 2010; 9: 63-.

$50 \mathrm{Hu}$ Z, Zhu,D, Wang,W, Li,W, Jia,W, Zeng,X et al. Genome-wide profiling of HPV integration in cervical cancer identifies clustered genomic hot spots and a potential microhomology-mediated integration mechanism. Nat Genet 2015; 47: 158-163.

51 Chia NY, Deng,N, Das,K, Huang,D, Hu,L, Zhu,Y et al. Regulatory crosstalk between lineage-survival oncogenes KLF5, GATA4 and GATA6 cooperatively promotes gastric cancer development. Gut 2014.

52 Xing C, Ci,X, Sun,X, Fu,X, Zhang,Z, Dong,EN et al. Klf5 deletion promotes Pten deletion-initiated luminal-type mouse prostate tumors through multiple oncogenic signaling pathways. Neoplasia 2014; 16: 883-899.

53 Zhang B, Zhang,Z, Xia,S, Xing,C, Ci,X, Li,X et al. KLF5 activates microRNA 200 transcription to maintain epithelial characteristics and prevent induced epithelialmesenchymal transition in epithelial cells. Mol Cell Biol 2013; 33: 4919-4935.

54 Tarapore RS, Yang,Y, Katz,JP. Restoring KLF5 in esophageal squamous cell cancer cells activates the JNK pathway leading to apoptosis and reduced cell survival. Neoplasia 2013; 15: 472-480.

55 Yang Y, Nakagawa,H, Tetreault,MP, Billig,J, Victor,N, Goyal,A et al. Loss of transcription factor KLF5 in the context of p53 ablation drives invasive progression of human squamous cell cancer. Cancer Res 2011; 71: 6475-6484.

56 Ton-That H, Kaestner,KH, Shields,JM, Mahatanankoon,CS, Yang,VW. Expression of the gut-enriched Kruppel-like factor gene during development and intestinal tumorigenesis. FEBS Lett 1997; 419: 239-243. 
57 Zhao W, Hisamuddin,IM, Nandan,MO, Babbin,BA, Lamb,NE, Yang,VW. Identification of Kruppel-like factor 4 as a potential tumor suppressor gene in colorectal cancer. Oncogene 2004; 23: 395-402.

58 Ghaleb AM, McConnell,BB, Nandan,MO, Katz,JP, Kaestner,KH, Yang,VW. Haploinsufficiency of Kruppel-like factor 4 promotes adenomatous polyposis coli dependent intestinal tumorigenesis. Cancer Res 2007; 67: 7147-7154.

59 Ghaleb AM, Aggarwal,G, Bialkowska,AB, Nandan,MO, Yang,VW. Notch inhibits expression of the Kruppel-like factor 4 tumor suppressor in the intestinal epithelium. Mol Cancer Res 2008; 6: 1920-1927.

60 Vaira V, Faversani,A, Martin,NM, Garlick,DS, Ferrero,S, Nosotti,M et al. Regulation of lung cancer metastasis by Klf4-Numb-like signaling. Cancer Res 2013; 73: 26952705.

61 Shum CK, Lau,ST, Tsoi,LL, Chan,LK, Yam,JW, Ohira,M et al. Kruppel-like factor 4 (KLF4) suppresses neuroblastoma cell growth and determines non-tumorigenic lineage differentiation. Oncogene 2013; 32: 4086-4099.

62 Huang Y, Chen,J, Lu,C, Han,J, Wang,G, Song,C et al. HDAC1 and Klf4 interplay critically regulates human myeloid leukemia cell proliferation. Cell Death Dis 2014; 5: e1491-.

63 Shi M, Cui,J, Du,J, Wei,D, Jia,Z, Zhang,J et al. A novel KLF4/LDHA signaling pathway regulates aerobic glycolysis in and progression of pancreatic cancer. Clin Cancer Res 2014; 20: 4370-4380.

64 Pandya AY, Talley,LI, Frost,AR, Fitzgerald,TJ, Trivedi,V, Chakravarthy,M et al. Nuclear localization of KLF4 is associated with an aggressive phenotype in earlystage breast cancer. Clin Cancer Res 2004; 10: 2709-2719.

65 Liu Z, Teng,L, Bailey,SK, Frost,AR, Bland,KI, Lobuglio,AF et al. Epithelial transformation by KLF4 requires Notch1 but not canonical Notch1 signaling. Cancer Biol Ther 2009; 8: 1840-1851.

$66 \mathrm{Yu}$ F, Li,J, Chen,H, Fu,J, Ray,S, Huang,S et al. Kruppel-like factor 4 (KLF4) is required for maintenance of breast cancer stem cells and for cell migration and invasion. Oncogene 2011; 30: 2161-2172. 
$67 \mathrm{Li} \mathrm{J}$, Zheng,H, Yu,F, Yu,T, Liu,C, Huang,S et al. Deficiency of the Kruppel-like factor KLF4 correlates with increased cell proliferation and enhanced skin tumorigenesis. Carcinogenesis 2012; 33: 1239-1246.

68 Cittelly DM, Finlay-Schultz,J, Howe,EN, Spoelstra,NS, Axlund,SD, Hendricks,P et al. Progestin suppression of miR-29 potentiates dedifferentiation of breast cancer cells via KLF4. Oncogene 2012; 32: 2555-2564.

69 Yu F, Shi,Y, Wang,J, Li,J, Fan,D, Ai,W. Deficiency of Kruppel-like factor KLF4 in mammary tumor cells inhibits tumor growth and pulmonary metastasis and is accompanied by compromised recruitment of myeloid-derived suppressor cells. Int J Cancer 2013; 133: 2872-2883.

70 Chen HF, Huang,CH, Liu,CJ, Hung,JJ, Hsu,CC, Teng,SC et al. Twist1 induces endothelial differentiation of tumour cells through the Jagged1-KLF4 axis. Nat Commun 2014; 5: 4697-.

71 Tien YT, Chang,MH, Chu,PY, Lin,CS, Liu,CH, Liao,AT. Downregulation of the KLF4 transcription factor inhibits the proliferation and migration of canine mammary tumor cells. Vet $J 2015$.

72 Orr N, Dudbridge,F, Dryden,N, Maguire,S, Novo,D, Perrakis,E et al. Fine-mapping identifies two additional breast cancer susceptibility loci at 9q31.2. Hum Mol Genet 2015.

73 Sharma SB, Lin,CC, Farrugia,MK, McLaughlin,SL, Ellis,EJ, Brundage,KM et al. microRNAs-206 and -21 cooperate to promote RAS-ERK signaling by suppressing the translation of RASA1 and SPRED1. Mol Cell Biol 2014.

74 Yori JL, Johnson,E, Zhou,G, Jain,MK, Keri,RA. Kruppel-like factor 4 inhibits epithelialto-mesenchymal transition through regulation of E-cadherin gene expression. $J$ Biol Chem 2010; 285: 16854-16863.

75 Yori JL, Seachrist,DD, Johnson,E, Lozada,KL, Abdul-Karim,FW, Chodosh,LA et al. Kruppel-like factor 4 inhibits tumorigenic progression and metastasis in a mouse model of breast cancer. Neoplasia 2011; 13: 601-610.

76 Kamalakaran S, Varadan,V, Giercksky Russnes,HE, Levy,D, Kendall,J, Janevski,A et al. DNA methylation patterns in luminal breast cancers differ from non-luminal 
subtypes and can identify relapse risk independent of other clinical variables. Mol Oncol 2011; 5: 77-92.

77 Dong MJ, Wang,LB, Jiang,ZN, Jin,M, Hu,WX, Shen,JG. The transcription factor KLF4 as an independent predictive marker for pathologic complete remission in breast cancer neoadjuvant chemotherapy: a case-control study. Onco Targets Ther 2014; 7: 1963-1969.

78 Duncan JS, Whittle,MC, Nakamura,K, Abell,AN, Midland,AA, Zawistowski,JS et al. Dynamic reprogramming of the kinome in response to targeted MEK inhibition in triple-negative breast cancer. Cell 2012; 149: 307-321.

79 Zhao Y, Hamza,MS, Leong,HS, Lim,CB, Pan,YF, Cheung,E et al. Kruppel-like factor 5 modulates p53-independent apoptosis through Pim1 survival kinase in cancer cells. Oncogene 2008; 27: 1-8.

80 Goard CA, Schimmer, AD. An evidence-based review of obatoclax mesylate in the treatment of hematological malignancies. Core Evid 2013; 8: 15-26.

81 Bialkowska A, Crisp,M, Madoux,F, Spicer,T, Knapinska,A, Mercer,B et al. ML264: An Antitumor Agent that Potently and Selectively Inhibits Kruppel-like Factor Five (KLF5) Expression: A Probe for Studying Colon Cancer Development and Progression. 2010. 


\section{Curriculum Vitae}

990 Northwestern Ave $\cdot$ Apt B $\cdot$ Morgantown, WV 26505

Phone (716) 830-7016·E-mail mfarrugia@hsc.wvu.edu

\section{Mark K Farrugia}

\section{Education}

2009-Present

-West Virginia University, Morgantown. Doctorate in Philosophy

expected 2015, Medical Doctorate expected 2017

2005-09

•Canisius College, Buffalo. Bachelor of Science, cum laude, Chemistry

\section{Research Experience}

2011-15 •MD/PhD trainee, Dept. of Biochemistry, WVU. Advisor: J. Michael Ruppert, $\mathrm{MD}, \mathrm{PhD}$. "Regulation of anti-apoptotic signaling by Kruppel-like factors 4 and 5 mediates lapatinib resistance in breast cancer"

2005-08 • Research Assistant, Dept. of Chemistry, Canisius College. "Highly

enantioselective cyclopropanation of substituted allenes using rhodium stabilized carbenoids"

\section{Publications}

Farrugia MK, Sharma,SB, Lin,CC, McLaughlin,SL, Vanderbilt,DB, Ammer,AG et al. Regulation of anti-apoptotic signaling by Kruppel-like factors 4 and 5 mediates lapatinib resistance in breast cancer. Cell Death Dis 2015; 6: e1699-.

Sharma SB, Lin,CC, Farnugia,MK, McLaughlin,SL, Ellis, EJ, Brundage,KM et al. microRNAs206 and -21 cooperate to promote RAS-ERK signaling by suppressing the translation of RASA1 and SPRED1. Mol Cell Biol 2014. Epub 2014 Sep 8 doi: 10.1128/MCB.00480-14.

Addison JB, Koontz,C, Fugett,JH, Creighton,CJ, Chen,D, Farnugia,MK et al. KAP1 promotes proliferation and metastatic progression of breast cancer cells. Cancer Res 2015; 75: 344-355. 
Timothy M Gregg, Mark K Farrugia, John R. Frost. Org. Lett., 2009, 11 (19), 4434-4436

Rhodium-Mediated Enantioselective Cyclopropanation of Allenes

Manuscripts in press

Lin,CC, Sharma SB, Farnugia,MK et al. Kruppel-like factor 4 signals through microRNA-206

to promote tumor initiation and cell survival. Oncogenesis 2015.

\section{Invited Reviews}

Farrugia MK, Ruppert JM. The role of KLF4 and KLF5 in cancer stem cell traits and chemoresistance. Cancer Res 2015. Under Review

\section{Presentations}

2015 • 'Regulation of anti-apoptotic signaling by Kruppel-like factors 4 and 5 mediates lapatinib resistance in breast cancer” Poster at Van Liere Research Day, WVU, Morgantown, WV

2014 • "Complementary and cooperative oncogenic signaling of KLF4 and KLF5 in breast cancer” Poster at Van Liere Research Day, WVU, Morgantown, WV

2014 - "Complementary and cooperative oncogenic signaling of KLF4 and KLF5 in breast cancer" Selected to give an oral presentation at Van Liere Research Day, WVU, Morgantown, WV

2013 • 'Relationship of KLF4 and KLF5 in breast cancer” Poster presentation at Van Liere Research Day, WVU, Morgantown, WV

2012-14 - Spoke to undergraduate students about admissions and career paths in science. WVU, Morgantown, WV

\section{Professional Honors \& Recognition}

2014 - Featured Graduate Student in the Mary Babb Randolph Cancer Center newsletter “Advances”, WVU, Morgantown, WV

- Awarded Best Poster Presentation at Van Liere Research Day, WVU, Morgantown, WV 
2011 - Recognition of Academic Excellence, WVU School of Medicine, Morgantown, WV

2009 - Merck Index Award, Canisius College, Buffalo, NY

\section{Academic Committees}

2012-2014 • MD/PhD Admissions Committee, WVU, Morgantown, WV

\section{Extra-curricular activities}

2012-present • MD/PhD program social chair. Plan outings/dinners to facilitate comradery within the students of the program. WVU, Morgantown, WV.

2010 -Habitat for Humanity. Over a week, I helped build a house in New Orleans for displaced victims of hurricane Katrina. New Orleans, LA 